\title{
20. EVOLUTION OF NORTHWEST PACIFIC SEDIMENTATION PATTERNS SINCE 6 MA (SITE 882) ${ }^{1}$
}

\author{
G.H. Haug, ${ }^{2}$ M.A. Maslin, ${ }^{2}$ M. Sarnthein, ${ }^{2}$ R. Stax,${ }^{3}$ and R. Tiedemann ${ }^{4}$
}

\begin{abstract}
A high-resolution carbonate record from the northwest Pacific covering roughly the last $6 \mathrm{~m} . y$. generally shows "Atlantic-type" interglacial spikes of carbonate preservation. These spikes parallel maxima in the biogenic opal and total organic carbon records, indicating increased productivity during interglacials. The variations can be explained best by changes in the advection of nutrientrich deep water originating in the North Atlantic and ultimately upwelling in the northwestern Pacific, the terminus of the global salinity conveyor belt. During interglacial times, the conveyor was enhanced, which led to increased upwelling of nutrient-rich deep water and, therefore, to a regionally enhanced surface productivity. As a result, carbonate production and accumulation at Site 882 exceeded the local carbonate dissolution during early/peak interglacial times, in contrast to the central Pacific where $\mathrm{CaCO}_{3}$ corrosion is characteristic of warm stages as a response to an increase in the circulation of the salinity conveyor belt, leading to the "Pacific type" of interglacial minima of carbonate accumulation.
\end{abstract}

\section{INTRODUCTION}

Site 882 provides the first high-resolution carbonate record in the North Pacific Ocean for the last 6 m.y. This site is located at the northern end of the Emperor Seamount Chain, on the western flank of the Detroit Seamount $\left(50^{\circ} 22^{\prime} \mathrm{N}, 167^{\circ} 36^{\prime} \mathrm{E}\right)$ in $3244 \mathrm{~m}$ water depth. The sedimentation rate at this site varied from 1.5 to $17 \mathrm{~cm} / \mathrm{k} . \mathrm{y}$, a rate sufficient to depict the oceanographic variability in the range of Milankovitch orbital forcing, provided a minimum sampling interval of $30 \mathrm{~cm}$. Changes in ocean carbonate preservation and/or dissolution yield important evidence for reconstructing the climate system as they record temporal variations in ocean biochemistry, which ultimately controls the atmospheric $\mathrm{CO}_{2}$ concentration. High-resolution records of gamma-ray attenuation porosity evaluator (GRAPE) density, magnetic susceptibility, $\mathrm{CaCO}_{3}$, and biogenic opal in conjunction with magnetostratigraphy (Rea, Basov, Janecek, Palmer-Julson, et al., 1993) have permitted an astronomical age calibration (Tiedemann and Haug, this volume) of the sediment record at Site 882 and the determination of fluxes. Detailed comparison were made between the carbonate and biogenic opal records from the North Pacific and with other highresolution sites from the Pacific and Atlantic oceans.

Several oceanographic studies have demonstrated that calcium carbonate preservation differs between the modern Atlantic and $\mathrm{Pa}$ cific Oceans (e.g., Broecker and Takahashi, 1978). The Atlantic is characterized by good carbonate preservation and a deep lysocline. In contrast, modern Pacific deep water is more corrosive to calcium carbonate, resulting in a shallow lysocline. This basin-to-basin fractionation is mainly attributed to the strong formation of oxygen-rich North Atlantic Deep Water (NADW). During its southward flow, the ongoing oxidation of settling organic matter causes a gradual increase in $\Sigma \mathrm{CO}_{2}$. After mixing with southern ocean water around Antarctica, this deep-water mass flows up to the North Pacific and becomes particularly further enriched with metabolic $\mathrm{CO}_{2}$ near the equator (Kroopnick, 1985) and is thus very corrosive to calcium carbonate.

A number of paleoceanographic studies have suggested for the last 4 m.y. that carbonate records from the Pacific and Atlantic are inversely correlated during glacial and interglacial times (e.g., Crowley,

\footnotetext{
${ }^{1}$ Rea, D.K., Basov, I.A., Scholl, D.W.. and Allan, J.F. (Eds.), 1995. Proc, ODP, Sci. Results, 145: College Station, TX (Ocean Drilling Program).

${ }^{2}$ Geologisch-Paläontologisches Institut, Universität Kiel, Olshausenstrasse 40 , D-24098 Kiel, Federal Republic of Germany.

${ }^{3}$ Alfred Wegener Institut für Polarforschung, Columbusstrasse, D-27568 Bremerhaven, Federal Republic of Germany.

${ }^{4}$ GEOMAR Forschungszentrum für Marine Geowissenschaften, Wischhofstrasse I-3, D-24148 Kiel, Federal Republic of Germany.
}

1985; Ruddiman, Sarnthein, et al., 1989; Farrell and Prell, 1991). The "Pacific-type" pattern is widely considered to be characterized by high carbonate contents during glacial times and low carbonate contents during interglacial times (Hebbeln et al., 1990; Farrell and Prell, 1991; Zahn et al., 1991; Karlin et al., 1992), whereas the "Atlantictype" pattern shows carbonate maxima during interglacials and minima during glacials (Olausson, 1965; Ruddiman, Sarnthein, et al., 1989). Temporal changes in deep-water chemistry and surface-water productivity appear to be major factors in controlling the different carbonate preservation patterns of the Atlantic and Pacific.

Apart from dilution, sedimentary calcium carbonate concentrations depend on $\mathrm{CaCO}_{3}$ flux rates (productivity) and dissolution rates on the seafloor. Enhanced carbonate preservation in the Pacific during glacial periods may suggest less "corrosive" North Pacific deepwater masses, as indicated by carbon isotope measurements (Curry et al., 1988; Duplessy et al., 1988) or by changes in the redox conditions in North Pacific sediments (Dean et al., 1989). Cadmium measurements on benthic foraminifers indeed suggest a glacial reduction of the nutrient concentration and hence a possible formation of deep water in the North Pacific (Boyle, 1992; Lynch-Stieglitz and Fairbanks, 1994). Maximum preservation cycles (23, 41, and $100 \mathrm{ka})$ in the equatorial Pacific lag minimum ice volume (low sea level) by about 6-20 k.y. and may be attributed to the response time of the carbonate system (Le and Shackleton, 1992) in the Pacific deep water. Hence, the glacial to interglacial carbonate cycles are out of phase with ice volume changes.

Productivity-induced changes in the organic carbon to $\mathrm{CaCO}_{3}$ rain ratio is the second main factor controlling the glacial/interglacial carbonate distribution patterns. Enhanced supply of $\mathrm{CaCO}_{3}$ deepens the carbonate compensation depth (CCD), whereas the concomitant flux of organic carbon and its oxidation increases $\mathrm{CaCO}_{3}$ dissolution (Berger and Keir, 1984; Archer, 1992; Berger, 1992; Le and Shackleton, 1992). Thus, $\mathrm{CaCO}_{3}$ mass accumulation rates (MARs) may increase if the $\mathrm{CaCO}_{3}$ production rate is rising at a greater rate than $\mathrm{C}_{\mathrm{org}}$ production and flux. For interpreting the $\mathrm{CaCO}_{3}$ concentration and MAR, the MAR of biogenic opal provides a strong argument for judging paleoproductivity vs. dissolution effects.

In this paper, we present a high-resolution carbonate record from the northwest Pacific covering roughly the last $6 \mathrm{~m} . \mathrm{y}$. and showing interglacial carbonate preservation peaks such as in the Atlantic. This feature was first shown by Hays and Shackleton (1976) and also reported by Keigwin et al. (1992) for the last glacial/interglacial cycle in the northwest Pacific. Moreover, Hovan et al. (1991) found greater $\mathrm{CaCO}_{3}$ accumulation during interglacial periods about $10^{\circ} \mathrm{S}$ of Site 882 before $350 \mathrm{ka}$. Dunn (1982) reported a change from "Atlantic- 
type" to "Pacific-type" carbonate stratigraphy in the middle Pliocene equatorial Pacific. He interpreted the timing of the change to the complete closure of the Isthmus of Panama that caused an increased mixing of NADW into Pacific bottom-water masses in the Circumpolar Water, due to an greater production of NADW during interglacials (Dunn, 1982).

\section{METHODS}

The $\mathrm{CaCO}_{3}$ contents (Table 1) were determined by coulometric technique using a Coulomat 702 at Kiel University. Furthermore, the carbonate record includes 143 shipboard values that were determined by the same technique. The accuracy of both data sets was better than $2 \%$, as obtained by replicate and standard measurements, and therefore, no offset between the two data sets is observed.

The total organic carbon contents (TOC) were measured using a Heraeus CHN-Analyzer at the Alfred-Wegener-Institut in Bremerhaven. To separate the carbonate bonded carbon from organic carbon the samples were treated with $\mathrm{HCL}(10 \%$, p.A.) and washed and dried. A detailed description of the analytical procedure is given in Stax and Stein (this volume).

The biogenic opal content (Table 2) was separated from the siliciclastic fraction in the carbonate-free fraction $>2 \mu \mathrm{m}$ by the density separation technique (Bohrmann et al., 1990), using sodium-polywolframate as the heavy liquid solution. The total reproducibility of the technique is roughly $\pm 5 \%$ for opal-poor sediments (less than $10 \%$ opal) and $\pm 3 \%$ of opal-rich sediments (over $30 \%$ opal) as indicated by replicate samples. However, a small systematic error must be considered from the separation of small silica fragments $(<2 \mu \mathrm{m})$ during the clay separation. The carbonate-free clay fraction was separated by means of the Atterberg separation method (Müller, 1967).

The siliciclastic fraction $>2 \mu \mathrm{m}$ (Table 2) was separated from the bulk sediment during the biogenic opal determination. It reflects in case of Site 882 a good first-order approximation for ice-rafted debris (IRD).

The $\delta^{18} \mathrm{O}$ values of the benthic and planktonic foraminifers (Table 3 ) were measured according to the standard techniques at the University of Kiel. The samples were freeze-dried and then wet sieved through a $63 \mu \mathrm{m}$ mesh sieve. The samples were then dry sieved at intervals of $150,250,315$, and $500 \mu \mathrm{m}$, from which the foraminifers were picked. One benthic and one planktonic species were selected for analysis: Uvigerina spp. and N. pachyderma (1). In cases in which the lack of calcium carbonate resulted in the measurement of only one specimen of Uvigerina spp. or a few specimens of $N$. pachyderma (1) (not less than 6), the gas pressure of the mass spectrometer was checked to be sure that there was sufficient material for a reliable estimate. In calcium carbonate rich intervals we measured 3 to 6 specimens of Uvigerina spp. and 20 specimens of $N$. pachyderma (I).

Mass accumulation rates $\left(\mathrm{g} / \mathrm{cm}^{2} \mathrm{k} . \mathrm{y}\right.$.) were calculated as the product of sedimentation rates $(\mathrm{cm} / \mathrm{k} . \mathrm{y}$.), percentages of the individual compounds, and dry bulk density values $\left(\mathrm{g} / \mathrm{cm}^{3}\right.$; Rea, Basov, Janecek, Palmer-Julson, et al., 1993). Mean sedimentation rates were linearly interpolated between age control points (Tiedemann and Haug, this volume). Dry-bulk density values were empirically derived from the gamma ray attenuation porosity evaluator (GRAPE) wet-bulk density analog data,

$$
\begin{gathered}
\text { Dry bulk density }=1.67 * \text { density GRAPE }-1.89, \\
(\mathrm{R}=0.83 ; N=112) .
\end{gathered}
$$

This equation is based on in situ dry-bulk density data from various depth sections throughout Hole $882 \mathrm{~A}$. The estimated drybulk density data reproduce the measured in situ data to within a standard error of $0.2 \mathrm{~g} / \mathrm{cm}^{3}$. The use of accumulation rates removes the effect of percentage dilution by different sediment components.

To obtain complete and continuous sedimentary records for the last $4.0 \mathrm{~m}$.y., we adjusted the depth of each sample to the composite depth record of Holes $882 \mathrm{~A}$ and $882 \mathrm{~B}$. The two records were spliced using correlations based on the magnetic susceptibility and GRAPE records of Holes $882 \mathrm{~A}$ and $882 \mathrm{~B}$ (Tiedemann and Haug, this volume). This composite depth scale was developed only for the upper 255.95 mbsf (Hole 882A), equivalent to $287.78 \mathrm{~m}$ composite depth. Further below, the composite depth section remains uncertain because the overlapping sections between Holes $882 \mathrm{~A}$ and $882 \mathrm{~B}$ are short and correlations are tenuous down to $270.4 \mathrm{mbsf}$, the core end of Hole 882B (Rea, Basov, Janecek, Palmer-Julson, et al., 1993).

\section{AGE MODEL}

The time scale at Site 882 was initially based on magnetostratigraphy (Rea, Basov, Janecek, Palmer-Julson, et al., 1993). The ages for the magnetic reversal boundaries were derived from the orbitally tuned time scale of Shackleton et al. $(1990,1995)$ and Tiedemann et al. (1994) improving on Hilgen (1991). From 2.6 to 6 Ma the tuned ages of the magnetic boundaries are about $1 \%-3 \%$ older than indicated by the time scale of Cande and Kent (1992). Using the astronomically dated magnetic reversals for initial age control, as expected the fluctuations in the GRAPE and magnetic susceptibility records were concentrated at periods near those of orbital eccentricity (400 and $100 \mathrm{ka}$ ), obliquity (41 ka), and precession (23 and $19 \mathrm{ka})$. To generate a high-resolution time scale for Site 882, the GRAPE-density record was fine-tuned back to $4 \mathrm{Ma}$ by adjusting the precession cycles to the summer insolation record at $65^{\circ} \mathrm{N}$ (Berger and Loutre, 1991). A detailed description of the astronomical calibrated age model using the standard techniques from Imbrie et al. (1984) is given by Tiedemann and Haug (this volume). Sedimentation rates were calculated by linear interpolation between adjusted age control points. The ages for the time interval 4 to $6 \mathrm{~m}$.y. are based on magnetostratigraphy (Rea, Basov, Janecek, Palmer-Julson, et al., 1993; Tiedemann and Haug, this volume).

\section{RESULTS}

The carbonate concentrations at Site 882 varied between 0 and 55 wt $\%$ over the last 6 m.y. There are major quasi-cyclic variations in amplitude, and many intervals have no carbonate. In general, Pleistocene interglacials and Pliocene warm stages have higher $\% \mathrm{CaCO}_{3}$ and higher $\mathrm{CaCO}_{3} \mathrm{MAR}$, which is comparable to the pattern of carbonate preservation in the Atlantic. In general, mass accumulation rates of $\mathrm{CaCO}_{3}$ show the same pattern as the wt $\% \mathrm{CaCO}_{3}$ (Figs. 1 and 2). Intervals with $\mathrm{CaCO}_{3}$ MAR up to $4 \mathrm{~g} / \mathrm{cm}^{2} \mathrm{k}$.y. occurred from 5.15 to $4.9 \mathrm{Ma}$ and from about 3.8 to $2.8 \mathrm{Ma}$. On the other hand, very low $\mathrm{CaCO}_{3}$ MARs prevailed over the rest of the record, with short-term and pronounced cyclic maxima of $\mathrm{CaCO}_{3}\left(0-1 \mathrm{~g} / \mathrm{cm}^{2} \mathrm{k}\right.$.y. $)$ intercalculated from 5.8 to $5.15 \mathrm{Ma}$ and from 1.7 Ma to the present (Figs. 1 and 2).

In comparison, biogenic opal MARs were continuously high (2-6 $\mathrm{g} / \mathrm{cm}^{2} \mathrm{k} . \mathrm{y}$.) from 3.2 to $2.73 \mathrm{Ma}$. The dramatic drop in opal MAR occurred at $2.73 \mathrm{Ma}$ (by a factor of 3 to 5 ), followed by a short period of low opal values until $2.25 \mathrm{Ma}$, a period with extremely low opal values ( $<0.3 \mathrm{~g} / \mathrm{cm}^{2} \mathrm{k} . \mathrm{y}$.) from 2.1 to $1.75 \mathrm{Ma}$, and finally low but distinct interglacial-to-glacial cycles $\left(0.1-1.5 \mathrm{~g} / \mathrm{cm}^{2} \mathrm{k} . \mathrm{y}\right.$.) until today, with high opal values generally linked to interglacial times (Fig. 2).

The extremely low TOC contents vary between 0 and $0.03 \mathrm{~g} / \mathrm{cm}^{2}$ k.y. The TOC maxima tend to follow the trends of the biogenic opal record (Fig. 2).

Over the last 2.7 m.y., maxima in biogenic opal generally parallel carbonate maxima. In addition, there are many opal maxima that occur during periods of low to zero $\mathrm{CaCO}_{3}$ accumulation. Within the Brunhes Chron, the carbonate accumulation ranges between 0 and 0.9 $\mathrm{g} / \mathrm{cm}^{2}$ k.y. During warm isotopic Stages $17,15,13$, and 11 , we found almost continuous carbonate accumulation. However, the $\mathrm{CaCO}_{3} \mathrm{MAR}$ in warm Stages 9,7,5, and 3 is discontinuous and occurs in punctuated spikes that are especially significant near Terminations 1 and 2 (Fig. 3). Hence, we conclude that the record resembles an "Atlantic-type" preservation history. 

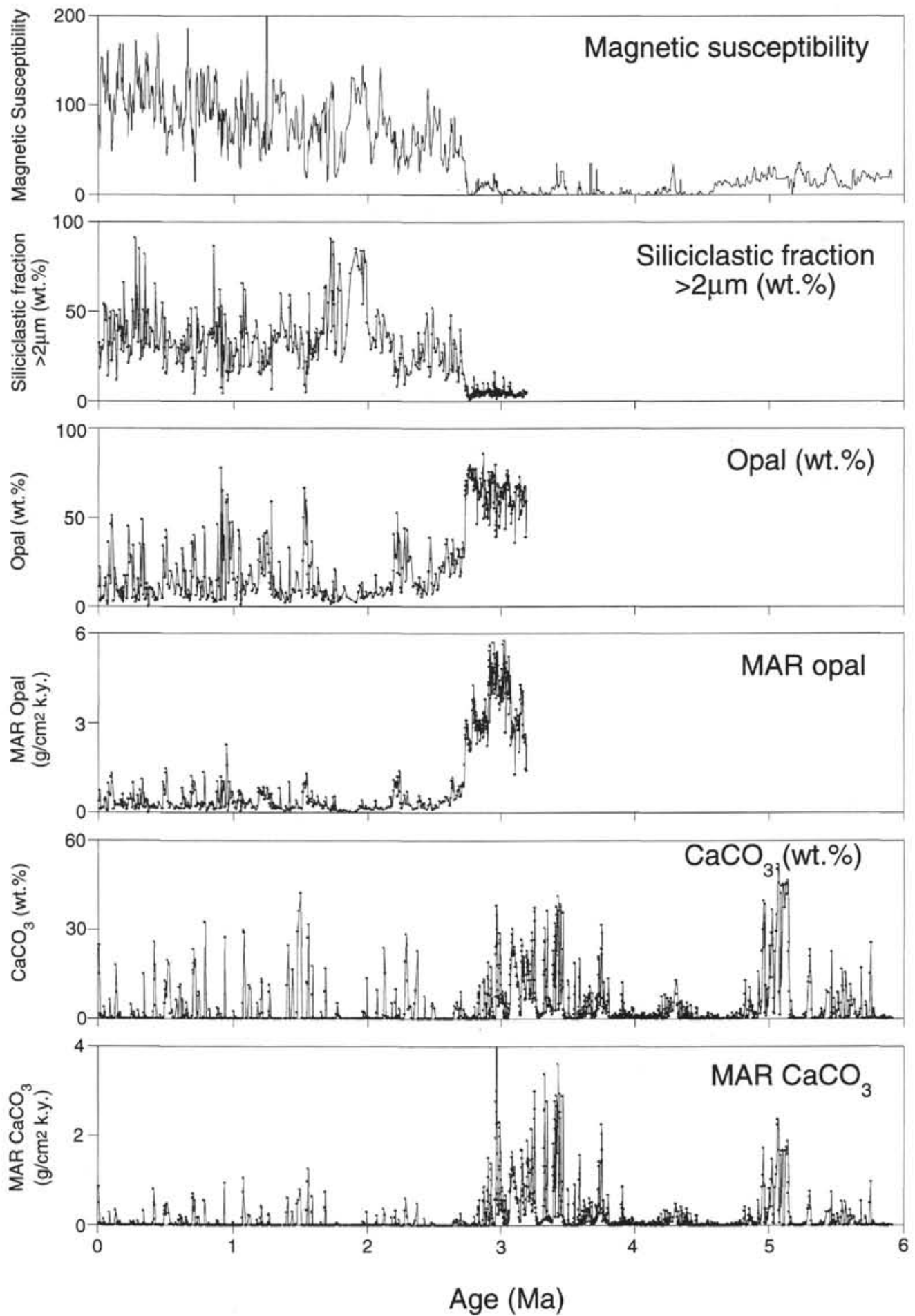

Figure 1. Summary of Site 882 magnetic susceptibility, siliciclastic faction $>2 \mu \mathrm{m}$ (wt $\%$ ), biogenic opal (wt $\%$ and $\mathrm{MAR}$ ), and $\mathrm{CaCO}_{3}$ (wt $\%$ and $\mathrm{MAR}$ ) data for the last $6 \mathrm{~m}$.y. The magnetic susceptibility and the siliciclastic fraction $>2 \mu \mathrm{m}$ indicate terrigenous input that is mainly ice-rafted detritus.

Note that the upper Miocene $\mathrm{CaCO}_{3}$ record closely resembles that of the Quaternary. The extreme $\mathrm{CaCO}_{3}$ preservation spike between 4.95 and 5.15 Ma may represent a response to the Mediterranean flooding event in the earliest Pliocene at 5.2 Ma (Müller and Mueller, 1991).

The major changes in the accumulation of $\mathrm{CaCO}_{3}$ and biogenic opal near 2.95-2.73 Ma are clearly coeval with the onset of major Northern Hemisphere glaciations. However, the response of carbonate and opal was different. The amplitudes of $\mathrm{CaCO}_{3}$ cycles gradually decreased over 250 k.y. In contrast, the biogenic opal percentages dropped in one major step exactly at $2.73 \mathrm{Ma}$, whereas the MAR dropped in two steps from 3 to $2.73 \mathrm{Ma}$.

Furthermore, the siliciclastic fraction $>2 \mu \mathrm{m}$ shows a good correlation with the magnetic susceptibility (Fig. 1). Both reflect mainly IRD input that rises dramatically at 2.75 m.y., during isotopic Stage G6 (Fig. 2). Therefore, the onset in IRD input occurs approximately 2 kyr earlier than the major change in opal accumulation. Pronounced cold stages 

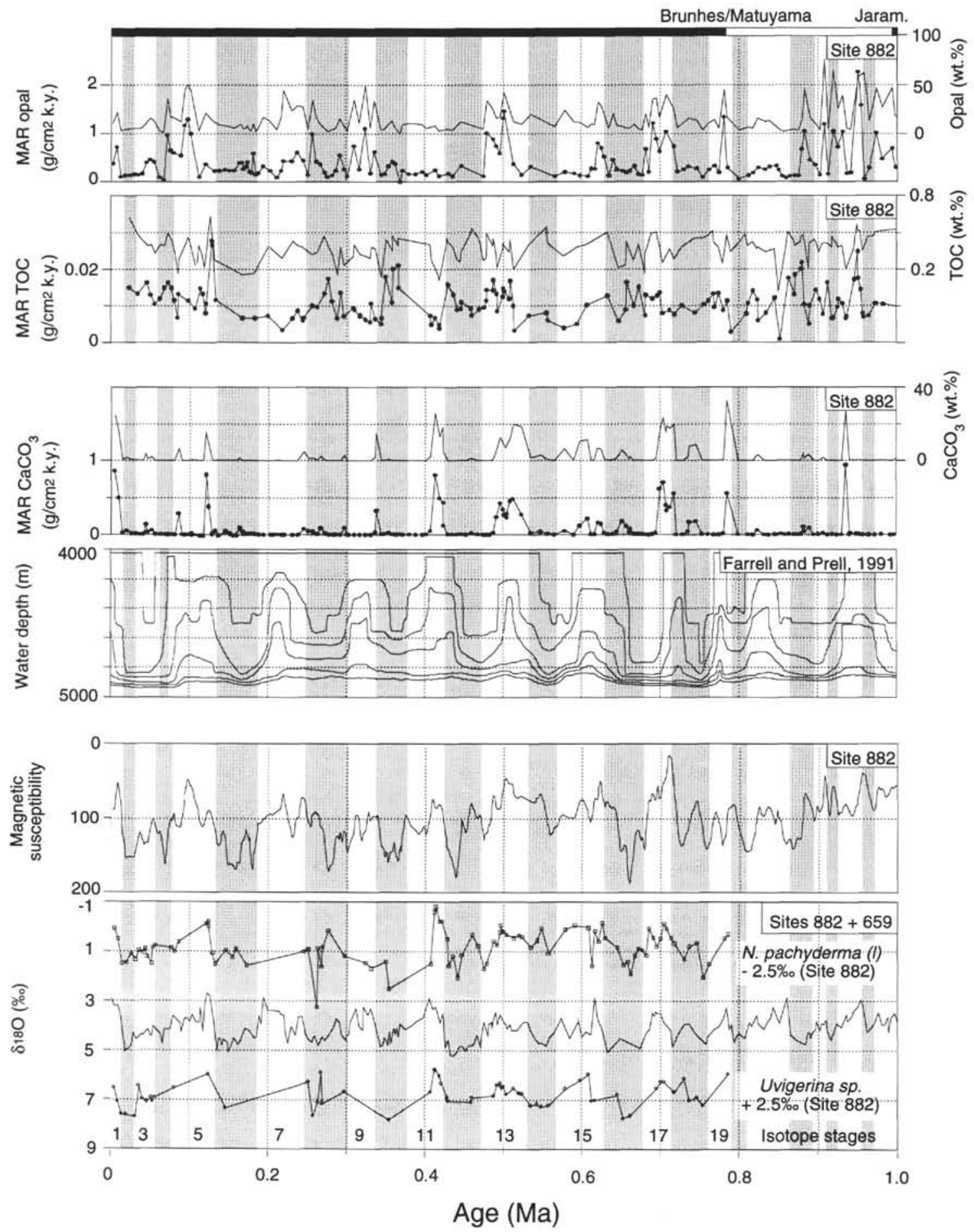

Figure 2. Mass accumulation rates and weight percentages of biogenic opal, $\mathrm{TOC}$ and $\mathrm{CaCO}_{3}$ for the last $6 \mathrm{~m} . \mathrm{y}$. of Site 882 in comparison to the equatorial Pacific bathymetric variations in $\mathrm{CaCO}_{3}$ preservation through time of the last 4 m.y. (Farrell and Prell, 1991). The age model of Farrell and Prell was updated to the new geomagnetic time scale of Hilgen (1991). Farrell and Prell plotted six isopleths of $90 \%$ (shallowest water depth), 80\%, 60\%, 40\%, 20\%, and 10\% (deepest water depth) carbonate level. The magnetic susceptibility record (reversed) is a good proxy for IRD input. Cold stages (shaded) are extrapolated from the global benthic oxygen isotope record from Site 659 (Tiedemann et al., 1994). 

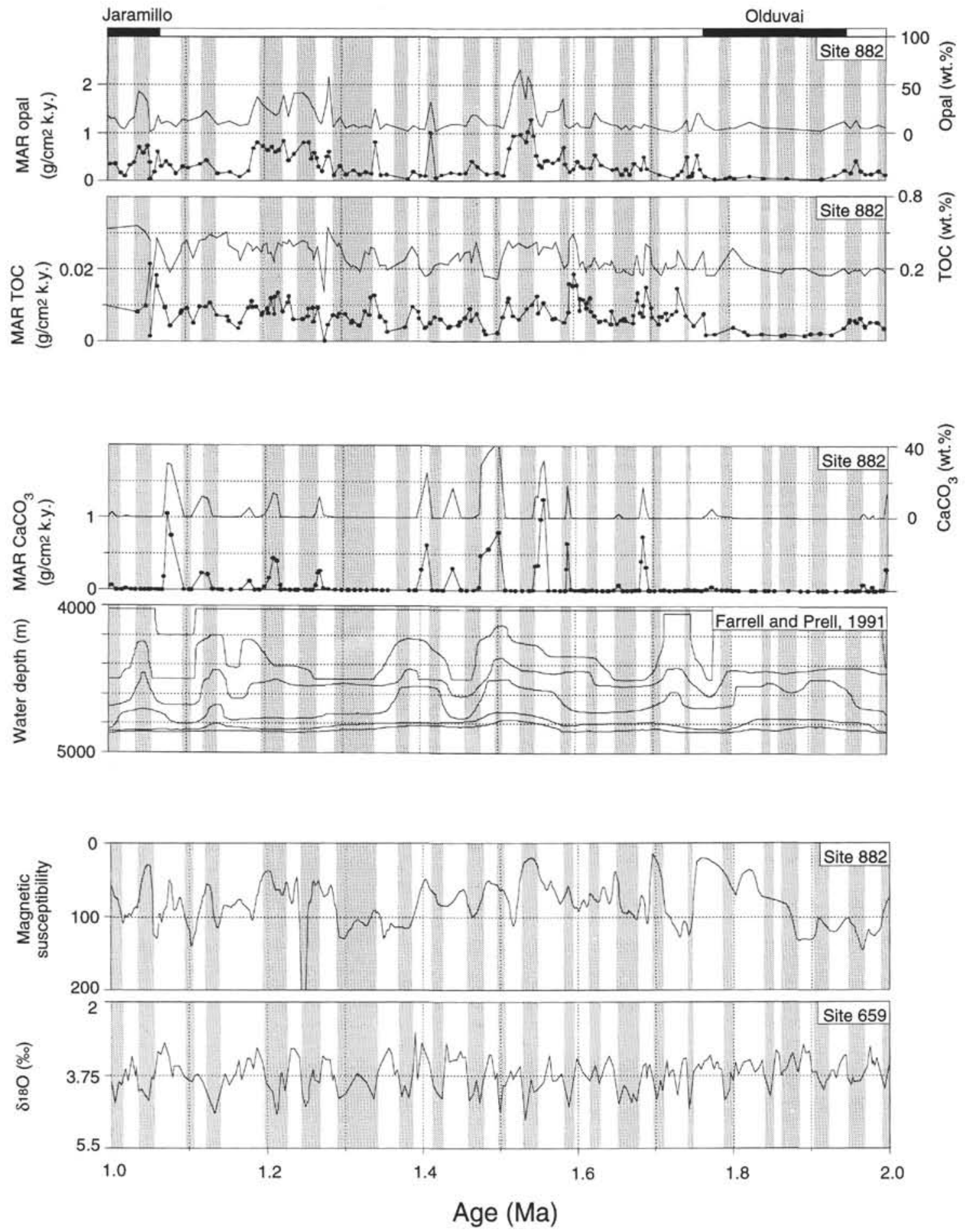

Figure 2 (continued).

like Stages 96, 98 and 100 are paralleled by dominant maxima in IRD input at Site 882. The concentrations of the siliciclastic fraction $>2$ $\mu \mathrm{m}$ varied between 4 and $90 \mathrm{wt} \%$ during the last $2.7 \mathrm{Ma}$.

The comparison of the global oxygen isotope stratigraphy with the low resolution isotope records from Site 882 corroborate the tuned age model of Site 882 (Tiedemann and Haug, this volume).

\section{DISCUSSION AND CONCLUSIONS}

The three factors controlling $\mathrm{CaCO}_{3}$ concentration are dilution (in this study excluded by calculated MAR), dissolution, and production
(Berger, 1992). The glacial carbonate peaks of the "Pacific type" carbonate pattern documented in the equatorial and Northeast Pacific (e.g., Farrell and Prell, 1991; Zahn et al., 1991; Karlin et al., 1992; Snoeckx and Rea, 1994) can be explained by a Pacific glacial intermediate deepwater source, evidence for which is given by carbon isotope (Curry et al., 1988; Duplessy et al., 1988) and paleo-nutrient measurements (Boyle, 1992; Lynch-Stieglitz and Fairbanks, 1994). A Pacific source of nutrient-depleted deep water would reduce the calcium carbonate corrosivity and therefore the carbonate dissolution during cold stages.

However, the opposite pattern with interglacial carbonate accumulation maxima recorded at Site 882 requires another mechanism. 

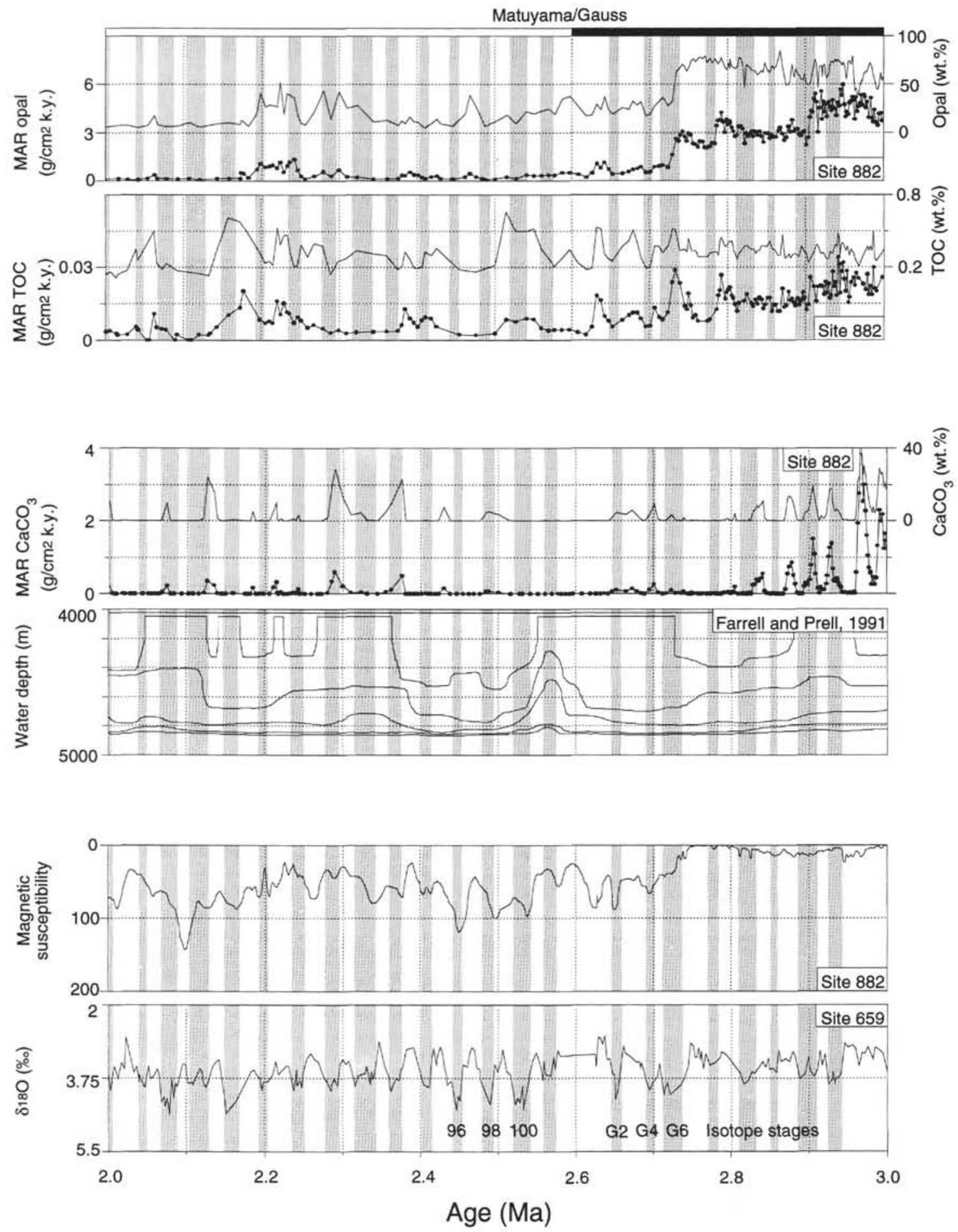

Figure 2 (continued).

We suggest a direct link to the global salinity conveyor belt, a mechanism in line to explain the carbonate patterns of the Atlantic, the Pacific and the inverse Pacific pattern at Site 882.

The impact of the two factors productivity and dissolution controlling $\mathrm{CaCO}_{3}$ concentration are clearly monitored at Site 882 , that is located close to the northwestern margin of the Pacific basin. In this corner of the Pacific, the global deep-water circulation that originates from North Atlantic sources reaches its terminus between the Emperor Seamount Chain and the Aleutian Arc, where the deep-water flow cannot continue horizontally but is funneled to the sea surface. This upwelling/upward diffusion is clearly depicted by GEOSECS data (Craig, 1981, see Fig. 3). Moreover, it can be detected in the reduced ${ }^{14} \mathrm{C}$ age differences between surface water and deep water at $3000 \mathrm{~m}$ (Broecker et al., 1988) as compared to the lower latitudes in the Pacific. Therefore, the northwest Pacific margin stands out as the main region of the upward-directed overturn in the global deep-water conveyor belt. The upwelled, very old North Atlantic-Pacific deep water which is enriched in $\mathrm{CO}_{2}$ and nutrients, influences $\mathrm{CaCO}_{3}$ deposition in two ways: (1) it is highly corrosive and dissolves carbonate on the seafloor as well as all over the Pacific basins; and (2) it triggers an unusually 

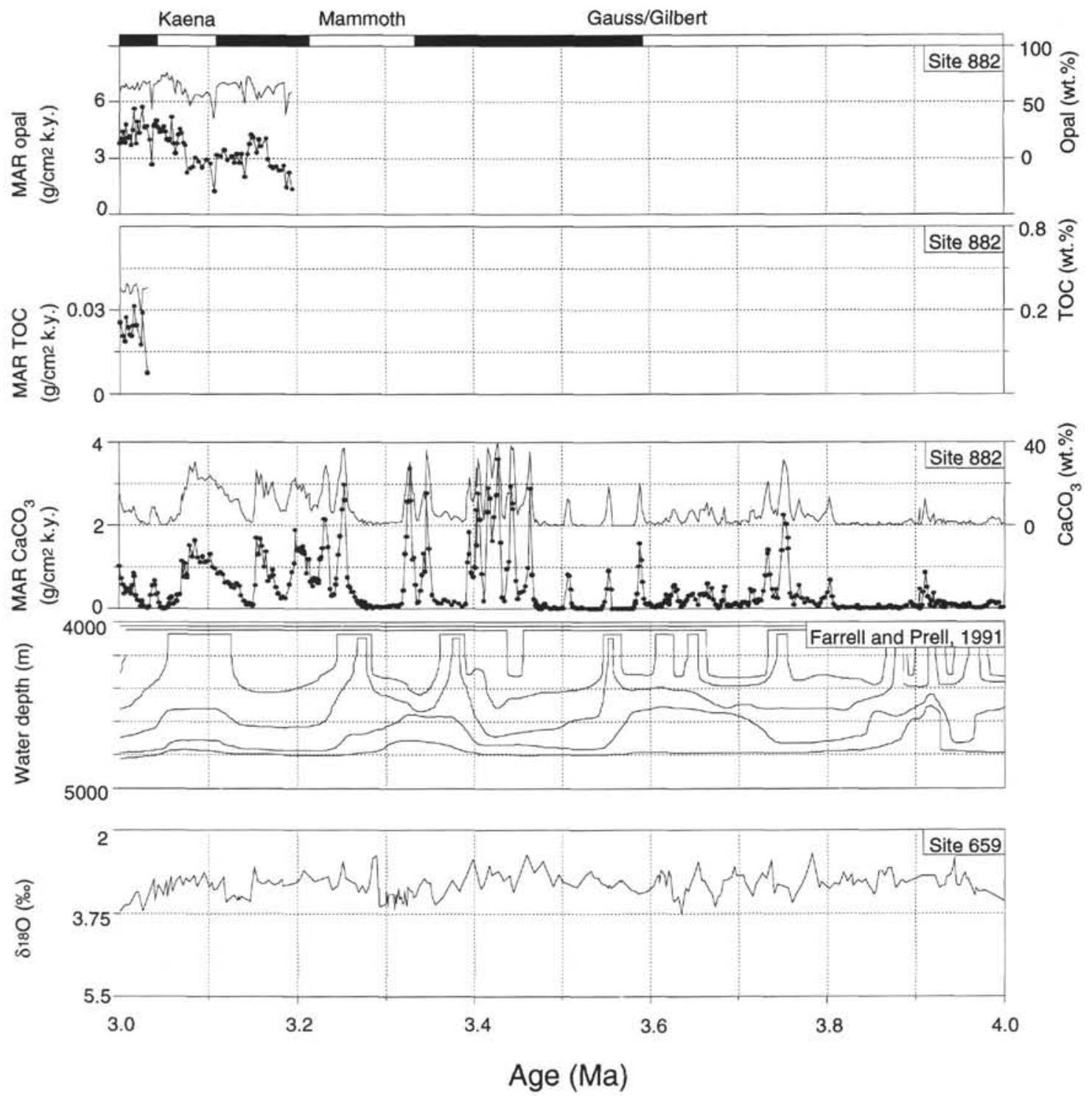

Figure 2 (continued).

high production of carbonate in the surface water, which may outweigh dissolution.

From this scenario, we conclude that the apparent "Atlantic-type" $\mathrm{CaCO}_{3}$ pattern at Site 882 must be dominated by the local productivity signal, in contrast to the "Pacific-type" $\mathrm{CaCO}_{3}$ records that occur in most other regions of the Pacific, where deep-water corrosion is the only response to an enhanced salinity conveyor belt. This interpretation of the Site 882 record is substantiated by parallel variations in the biogenic opal and TOC records that generally demonstrate that there was much higher production during Quaternary interglacials than during glacials.

In summary, such variations can be best explained by changes in the advection of nutrient enriched ocean deep-water upwelling in the northwest corner of the Pacific (Fig. 4). It is well documented that the formation of NADW culminated during interglacial times and was reduced during glacial phases (Duplessy et al., 1988; Sarnthein et al., 1994) (Fig. 4A). Therefore, the whole salinity conveyor belt circulation is stronger resulting in a well-ventilated, oxygen-enriched, nutrient-depleted and less $\mathrm{CaCO}_{3}$ corrosive deep-water masses in the Atlantic ("Atlantic-type") (Fig. 4B). In contrast, enhanced interglacial conveyor belt circulation leads in the Pacific to a higher input of nutrient- and $\mathrm{CO}_{2}$-enriched, highly $\mathrm{CaCO}_{3}$ corrosive deep-water masses, resulting in interglacial dissolution maxima in the equatorial and northeast Pacific ("Pacific-type") (Fig. 4C). At the terminus of the conveyor belt in the NW-Pacific, enhanced interglacial circulation of the whole conveyor belt system requires a higher upward diffusion of old and nutrient-enriched Pacific deep water. The higher productivity outweighs the high Pacific $\mathrm{CaCO}_{3}$ dissolution culminating in interglacial carbonate accumulation maxima ("Atlantic-type pattern at Site 882") (Fig. 4D).

Shackleton et al. (1988) showed that the Pacific deep water took $500 \mathrm{yr}$ longer to ventilate during the last glacial maximum than today and, accordingly, that there must have been a corresponding reduction in the amount of water upwelled in the northwest Pacific as monitored at Site 882 .

This change may have important implications for the atmospheric $\mathrm{CO}_{2}$ changes in the past. Tans et al. (1991) demonstrated that for the modern western subarctic Pacific is a site of significant $\mathrm{CO}_{2}$ outgassing, presumably from the upwelling deep water. On the other hand, the reduction in glacial northwest Pacific upwelling must have led to a great reduction in $\mathrm{CO}_{2}$ outgassing and, hence, in atmospheric $\mathrm{CO}_{2}$ (as documented in the Vostok ice core; Jouzel et al., 1993; Barnola et 

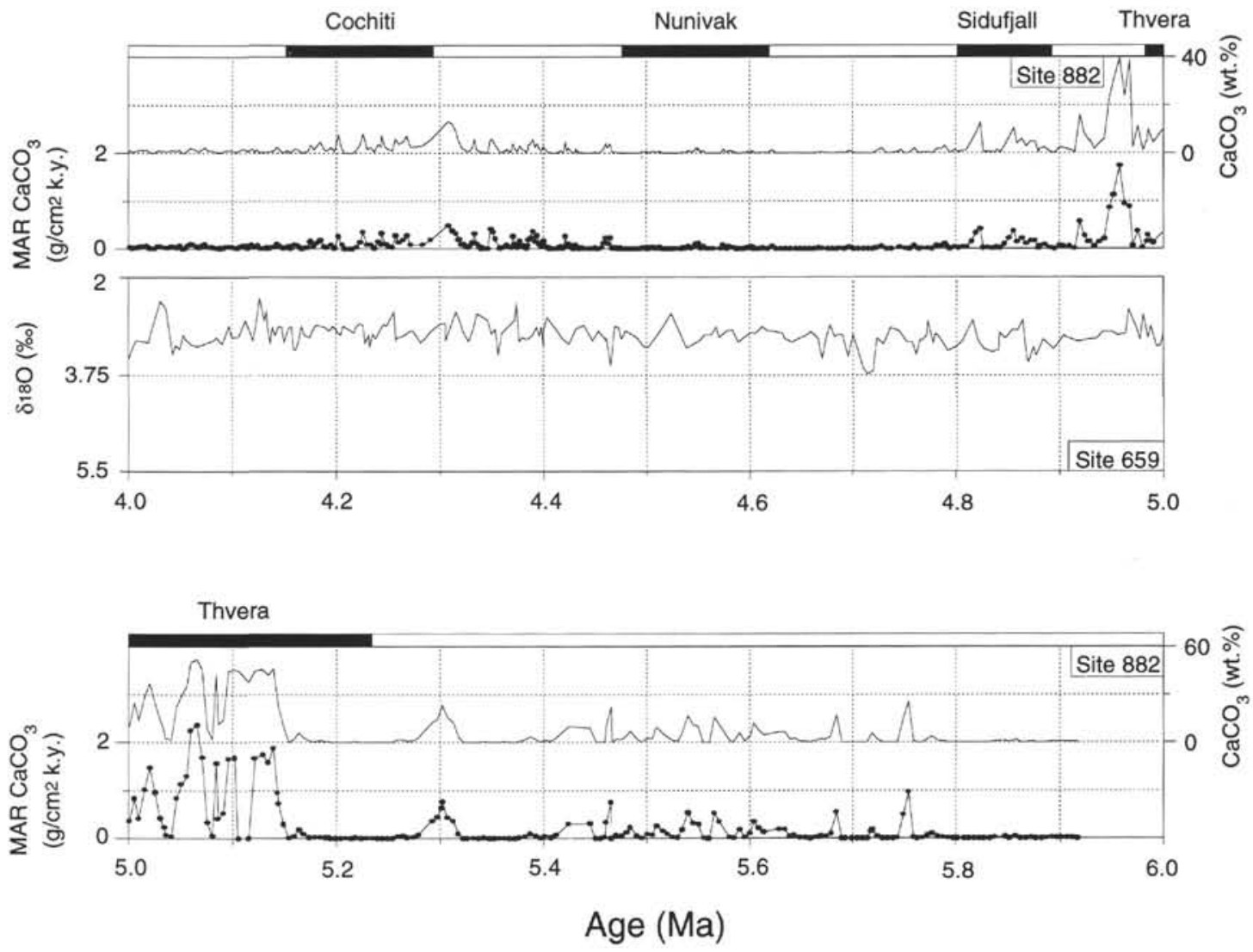

Figure 2 (continued).

al., 1987). This conclusion is in line with the $\mathrm{CaCO}_{3}$ records from the low-latitude Pacific (Fig. 2; Farrell and Prell, 1991), which show a clear decrease in $\mathrm{CaCO}_{3}$ dissolution during glacial times when there was no deep-water-induced $\mathrm{CaCO}_{3}$ productivity signal at Site 882 .

Finally, this reduction in Atlantic to Pacific deep-water supply and nutrient-rich upwelling in the glacial northwest Pacific may provide a plausible explanation for the obvious glacial Cd-depletion observed by Boyle (1992). If this mechanism of reduced upwelling and nutrient supply is substantiated, there is no more need for a glacial source of deep water in the northwest Pacific.

The conceptual model of considering Site 882 as a monitor of variations at the far end of the global deep-water conveyor belt may be also valid for understanding the late Neogene $\mathrm{CaCO}_{3}$, TOC, and opal records. In this view, the high carbonate accumulation spikes from 2.75 to $3.8 \mathrm{Ma}$ and from 4.8 to $5.2 \mathrm{Ma}$ (paralleled by strong dissolution events in the equatorial Pacific; Fig. 2) may reflect periods of significantly enhanced NADW production toward the end of the middle Pliocene climatic optimum that were possibly induced by the gradual closure of the Panama ocean connection (Keller et al., 1989; Mann and Corrigan, 1990) and interestingly, near the beginning of the Pliocene. This age of about $5.2 \mathrm{Ma}$ directly corresponds to the post Messinian flooding of the Mediterranean Sea (4.85/4.90 Ma on the conventional time scale; Müller and Mueller, 1991; McKenzie et al., 1990) and to a widespread hiatus in the Atlantic sediment profiles (Ruddiman, Sarnthein, et al., 1989).

In addition, the decrease in productivity during Quaternary cold stages at Site 882 may also be related to the southward advances of the subarctic sea ice margin, accompanied by a reduction in the supply of nutrients due to a weak Kamchatka-Oyashio current (Thompson and Shackleton, 1980; Sancetta, 1981).

However, we have no idea yet of (1) how to explain the origin of the dramatic and irreversible breakdown in biogenic opal production at 2.73 Ma (Maslin et al., this volume) and (2) the anti-correlation in the carbonate patterns between the northwest and northeast Pacific during the Quaternary.

\section{ACKNOWLEDGMENTS}

The authors thank J. Farrell whose comments greatly improved the manuscript. We gratefully acknowledge the cooperation with $\mathrm{H}$. Erlenkeuser and $\mathrm{H}$. Cordt, who provided the isotope data at the mass spectrometer in Kiel with special care. We thank E. Heinrich, J. Hennings, and C. Hohnemann for technical assistance. The Deutsche Forschungsgemeinschaft generously supported this study.

\section{REFERENCES}

Archer, D.E., 1991. Equatorial Pacific calcite preservation cycles: production or dissolution? Paleoceanography, 6:561-571.

Barnola, J.M., Raynaud, D., Korotkevich, Y.S., and Lorius, C., 1987. Vostok ice core provides 160,000-year record of atmospheric $\mathrm{CO}_{2}$. Nature, 329: $408-414$.

Berger, A., and Loutre, M.F., 1991. Insolation values for the climate of the last 10 million years. Quat. Sci. Rev., 10:297-317.

Berger, W.H., 1992. Pacific carbonate cycles revisited: arguments for and against productivity control. In Ishizaki, K., and Saito, T. (Eds.), Centenary of Japanese Micropaleontology: Tokyo (Terra Sci.), 15-25.

Berger, W.H., and Keir, R.S., 1984. Glacial-Holocene changes in atmospheric $\mathrm{CO}_{2}$ and the deep-sea record. In Hansen, J.E., and Takahashi, T. (Eds.), Climate Processes and Climate Sensitivity. Geophys. Monogr., Maurice Ewing Ser. 5, Am. Geophys. Union, 29:337-351.

Abbreviations for names of organizations and publications in ODP reference lists follow the style given in Chemical Abstracts Service Source Index (published by American Chemical Society). 
Bohrmann, G., Henrich, R., and Thiede, J., 1990. Miocene to Quaternary paleoceanography in the northern North Atlantic: variability in changes in carbonate and biogenic opal accumulation. In Bleil, U., and Thiede, J. (Eds.), Geological History of the Polar Oceans: Arctic versus Antarctic. NATO ASI Series C, 647-675.

Boyle, E.A., 1992. Cadmium and $\delta^{13} \mathrm{C}$ paleochemical ocean distributions during the stage 2 glacial maximum. Annu. Rev. Earth Planet. Sci., 20:245-287.

Broecker, W.S., Andree, M., Bonani, G., Wolfli, W., Oeschger, H., Klas, M., Mix, A., and Curry, W., 1988. Preliminary estimates for the radiocarbon age of deep water in the glacial ocean. Paleoceanography, 3:659-669.

Broecker, W.S., Peng, T.-H., Trumbore, S.E., Bonani, G., and Wolfli, W., 1990 The distribution of radiocarbon in the glacial ocean. Global Biogeochem. Cycles, 4:103-117.

Broecker, W.S., and Takahashi, T., 1978. The relationship between lysocline depth and in-situ carbonate ion concentration. Deep-Sea Res. Part A, 25:65-95.

Cande, S.C., and Kent, D.V., 1992. A new geomagnetic polarity time scale for the Late Cretaceous and Cenozoic. J. Geophys. Res., 97:13917-13951.

Craig, H., Broecker, W.S., and Spencer, D., 1981. GEOSECS Pacific Expedition (Vol. 4): Sections and Profiles: Washington (National Science Foundation).

Crowley, T.J., 1985. Late Quaternary carbonate changes in the North Atlantic and Atlantic/Pacific comparisons. In Sundquist, E.T., and Broecker, W.S (Eds.), The Carbon Cycle and Atmospheric $\mathrm{CO}_{2}$ : Natural Variations, Archean to Present. Geophys. Monogr., Am. Geophys. Union, 32:271-284.

Curry, W.B., Duplessy, J.-C., Labeyrie, L.D., Shackleton, N.J., 1988. Changes in the distribution of $\delta^{13} \mathrm{C}$ of glacial deep water $\Sigma \mathrm{CO}_{2}$ between the last glaciation and the Holocene. Paleoceanography, 3:317-341.

Dean, W.E., Gardner, J.V., and Hemphill-Haley, E., 1989. Changes in redox conditions in deep-sea sediments of the Subarctic North Pacific Ocean: possible evidence for the presence of North Pacific Deep Water. Paleoceanography, 4:639-653.

Dunn, D.A., 1982. Change from "Atlantic-type" to "Pacific type" carbonate stratigraphy in the middle Pliocene equatorial Pacific Ocean. Mar. Geol., 50:41-60.

Duplessy J.-C., Shackleton, N.J., Fairbanks, R.G., Labeyrie, L., Oppo, D., and Kallel, N., 1988. Deep-water source variations during the last climatic cycle and their impact on global deepwater circulation. Paleoceanography, 3:343-360.

Farrell, J.W., and Prell, W.L., 1991. Pacific $\mathrm{CaCO}_{3}$ preservation and $\delta^{18} \mathrm{O}$ since $4 \mathrm{Ma}$ : paleoceanic and paleoclimatic implications. Paleoceanography, 6:485-498.

Hays, J.D., and Shackleton, N.J., 1976. Globally synchronous extinction of the radiolarian Stylatractus universus. Geology, 4:649-652.

Hebbeln, D., Wefer, G., and Berger, W.H., 1990. Pleistocene dissolution fluctuations from apparent depth of deposition in Core ERDC-127P, westequatorial Pacific. Mar. Geol., 92:165-176.

Hilgen, F.J., 1991. Extension of the astronomically calibrated (polarity) time scale to the Miocene/Pliocene boundary. Earth Planet. Sci. Lett., 107:349368.

Hovan, S.A., Rea, D.K., and Pisias, N.G., 1991. Late Pleistocene continental climate and oceanic variability recorded in northwest Pacific sediments. Paleoceanography, 6:349-370.

Imbrie, J., Hays, J.D., Martinson, D.G., McIntyre, A., Mix, A.C., Morley, J.J., Pisias, N.G., Prell, W.L., and Shackleton, N.J., 1984. The orbital theory of Pleistocene climate: support from a revised chronology of the marine $\delta^{18} \mathrm{O}$ record. In Berger, A., Imbrie, J., Hays, J., Kukla, G., and Saltzman, B. (Eds.), Milankovitch and Climate (Pt. 1): Dordrecht (D. Reidel), 269-305.

Jouzel, J., Barkov, N.I., Barnola, J.M., Bender, M., Chappellaz, J., Genthon, C., Kotlyakov, V.M., Lipenkov, V., Lorius, C., Petit, J.R., Raynaud, D., Raisbeck, G., Ritz, C., Sowers, T., Stievenard, M., Yiou, F., and Yiou, P., 1993. Extending the Vostok ice-core record of palaeoclimate to the penultimate glacial period. Nature, 364:407-412.

Karlin, R., Lyle, M., and Zahn, R., 1992. Carbonate variations in the Northeast Pacific during the late Quaternary. Paleoceanography, 7:43-61.

Keigwin, L.D., Jones, G.A., and Froelich, N.P., 1992. A 15,000-year paleoenvironmental record from Meiji seamount, far northwestern Pacific. Earth Planet. Sci. Lett., 111:425-440.
Keller, G., Zenker, C.E., and Stone, S.M., 1989. Late Neogene history of the Pacific-Caribbean gateway. J. South Am. Earth Sci., 2:73-108.

Kroopnick, P., 1985. The distribution of ${ }^{13} \mathrm{C}$ of $\Sigma \mathrm{CO}_{2}$ in the world oceans. Deep-Sea Res. Part A, 32:57-84.

Le, J., and Shackleton, N.J., 1992. Carbonate dissolution fluctuations in the western equatorial Pacific during the late Quaternary. Paleoceanography, $7: 21-42$.

Lynch-Stieglitz, J., and Fairbanks, R.G., 1994. A conservative tracer for glacial ocean circulation from carbon isotope and paleo-nutrient measurements in benthic foraminifera. Nature, 369:308-310.0

Mann, P., and Corrigan, J., 1990. Model of late Neogene deformation in Panama. Geology, 18:558-562.

McKenzie, J.A., Sprovieri, R., and Channel, J.E.T., 1990. The terminal Messinian flood and earliest Pliocene paleoceanography in the Mediterranean: results from ODPLeg 107, Site 652, Tyrrhenian Sea.Mem. Soc. Geol. Ital., 44:81-91.

Müller, D.W., and Mueller, P.A., 1991. Origin and age of the Messinian evaporites: implications from Sr isotopes. Earth Planet. Sci. Lett., 107:1-12.

Müller, G., 1967. Methods in sedimentary petrology. In Von Engelhardt, W., Füchtbauer, H., and Müller, G. (Eds.), Sedimentary Petrology (Pt. 1): Stuttgart (Schweitzerbart'sche Verlagsbuchhandlung).

Olausson, E., 1965. Evidence of climatic changes in north Atlantic deep sea cores, with remarks on isotopic paleotemperature analysis. Progr. Oceanogr, 3:221-252.

Rea, D.K., Basov, I.A., Janecek, T.R., Palmer-Julson, A., et al., 1993. Proc. ODP, Init. Repts., 145: College Station, TX (Ocean Drilling Program).

Ruddiman, W., Sarnthein, M., et al., 1989. Proc. ODP, Sci. Results, 108: College Station, TX (Ocean Drilling Program).

Sancetta, C., 1981. Oceanographic and ecologic significance of diatoms in surface sediments of the Bering and Okhotsk seas. Deep-Sea Res. Part A, 28:789-817.

Sarnthein, M., Winn, K., Jung, S., Duplessy, J.-C., Labeyrie, L., Erlenkeuser, H., and Ganssen, G., 1994. Changes in East Atlantic deep-water circulation over the last 30,000 years: eight-time-slice reconstructions. Paleoceanography, 9:209-267.

Shackleton, N.J., Berger, A., and Peltier, W.R., 1990. An alternative astronomical calibration of the lower Pleistocene timescale based on ODP Site 677. Trans. R. Soc. Edinburgh: Earth Sci., 81:251-261.

Shackleton, N.J., Duplessy, J.-C., Arnold, M., Maurice, P., Hall, M.A., and Cartlidge, J., 1988. Radiocarbon age of the last glacial Pacific deep water. Nature, 235:708-711.

Shackleton, N.J., Hall, M.A., and Pate, D., 1995. Pliocene stable isotope stratigraphy of Site 846. In Pisias, N.G., Mayer, L.A., Janecek, T.R., Palmer-Julson, A., and van Andel, T.H. (Eds.), Proc. ODP, Sci. Results, 138: College Station, TX (Ocean Drilling Program), 337-356.

Snoecks, H., and Rea, D.K., 1994. Late Quaternary $\mathrm{CaCO}_{3}$ stratigraphy of the eastern equatorial Pacific. Paleoceanography, 9:341-353.

Tans, P.P., Fung, I.Y., and Takahashi, T., 1990. Observational constraints on the global atmospheric $\mathrm{CO}_{2}$ budget. Science, 247:1431-1436.

Thompson, P.R., and Shackleton, N.J., 1980. North Pacific paleoceanography: late Quaternary coiling variations of planktonic foraminifer Neogloboquadrina pachyderma. Nature, 287:829-833.

Tiedemann, R., Sarnthein, M., and Shackleton, N.J., 1994. Astronomic timescale for the Pliocene Atlantic $\delta^{13} \mathrm{C}$ and dust flux records of ODP Site 659. Paleoceanography, 9:619-638.

Zahn, R., Rushdi, A., Pisias, N.G., Bornhold, B.D., Blaise, B., and Karlin, R., 1991. Carbonate deposition and benthic $\delta^{13} \mathrm{C}$ in the subarctic Pacific: implications for changes of the oceanic carbonate system during the past 750,000 years. Earth Planet. Sci. Lett., 103:116-132.

Date of initial receipt: 11 April 1994

Date of acceptance: 7 October 1994

Ms 145SR-115 
Table 1. $\mathrm{CaCO}_{3}$ data from Site 882 .

\begin{tabular}{|c|c|c|c|}
\hline $\begin{array}{l}\text { Composite } \\
\text { depth } \\
\text { (m) }\end{array}$ & $\begin{array}{l}\text { Age } \\
\text { (ka) }\end{array}$ & $\begin{array}{l}\mathrm{CaCO}_{3} \\
\text { (wt\%) }\end{array}$ & $\begin{array}{c}\text { MAR } \\
\mathrm{CaCO}_{3} \\
\left(\mathrm{~g} / \mathrm{cm}^{2} \mathrm{k} . \mathrm{y} .\right)\end{array}$ \\
\hline 0.30 & 4.9 & 24.70 & 0.86 \\
\hline 0.60 & 9.8 & 15.15 & 0.49 \\
\hline 0.90 & 14.7 & 0.77 & 0.02 \\
\hline 1.20 & 20.1 & 1.61 & 0.05 \\
\hline 1.55 & 26.4 & 0.47 & 0.01 \\
\hline 1.85 & 31.9 & 0.51 & 0.02 \\
\hline 2.10 & 36.4 & 0.11 & 0.00 \\
\hline 2.36 & 41.1 & 0.25 & 0.01 \\
\hline 2.55 & 44.6 & 4.00 & 0.14 \\
\hline 2.66 & 46.6 & 0.92 & 0.03 \\
\hline 2.96 & 52.0 & 1.96 & 0.06 \\
\hline 3.20 & 56.4 & 0.15 & 0.00 \\
\hline 3.56 & 62.9 & 0.12 & 0.00 \\
\hline 3.86 & 68.4 & 0.08 & 0.00 \\
\hline 4.05 & 71.5 & 0.00 & 0.00 \\
\hline 4.16 & 73.1 & 0.06 & 0.00 \\
\hline 4.46 & 77.4 & 0.06 & 0.00 \\
\hline 4.70 & 80.9 & 0.04 & 0.00 \\
\hline 5.06 & 86.1 & 6.52 & 0.28 \\
\hline 5.36 & 90.5 & 0.08 & 0.00 \\
\hline 5.55 & 93.3 & 0.00 & 0.00 \\
\hline 5.66 & 94.9 & 0.07 & 0.00 \\
\hline 5.96 & 99.2 & 0.09 & 0.00 \\
\hline 6.20 & 102.7 & 0.68 & 0.02 \\
\hline 6.86 & 113.4 & 0.09 & 0.00 \\
\hline 7.05 & 118.1 & 0.00 & 0.00 \\
\hline 7.16 & 122.0 & 0.05 & 0.00 \\
\hline 7.46 & 132.5 & 18.14 & 0.34 \\
\hline 7.70 & 139.1 & 8.71 & 0.22 \\
\hline 8.06 & 146.1 & 0.61 & 0.02 \\
\hline 8.36 & 151.9 & 1.36 & 0.04 \\
\hline 8.66 & 157.7 & 0.09 & 0.00 \\
\hline 9.20 & 164.4 & 2.03 & 0.09 \\
\hline 9.49 & 167.4 & 1.03 & 0.04 \\
\hline 9.69 & 169.4 & 0.15 & 0.01 \\
\hline 9.93 & 171.9 & 0.17 & 0.01 \\
\hline 10.17 & 174.4 & 0.13 & 0.01 \\
\hline 10.45 & 177.3 & 0.18 & 0.01 \\
\hline 10.68 & 179.7 & 0.15 & 0.01 \\
\hline 10.95 & 182.5 & 0.08 & 0.00 \\
\hline 11.17 & 184.7 & 0.06 & 0.00 \\
\hline 11.42 & 187.9 & 0.05 & 0.00 \\
\hline 11.64 & 194.3 & 0.07 & 0.00 \\
\hline 11.94 & 203.2 & 0.09 & 0.00 \\
\hline 12.06 & 206.7 & 0.12 & 0.00 \\
\hline 12.23 & 211.7 & 0.18 & 0.00 \\
\hline 12.41 & 217.0 & 0.14 & 0.00 \\
\hline 12.52 & 220.5 & 0.06 & 0.00 \\
\hline 12.81 & 230.7 & 0.05 & 0.00 \\
\hline 13.10 & 237.8 & 0.05 & 0.00 \\
\hline 13.40 & 245.1 & 4.81 & 0.08 \\
\hline 13.65 & 251.2 & 1.45 & 0.05 \\
\hline 13.97 & 256.9 & 1.31 & 0.04 \\
\hline 14.27 & 262.2 & 0.30 & 0.01 \\
\hline 14.56 & 267.4 & 2.24 & 0.09 \\
\hline 14.86 & 272.7 & 0.43 & 0.02 \\
\hline 15.07 & 276.4 & 0.09 & 0.00 \\
\hline 15.14 & 277.6 & 0.00 & 0.00 \\
\hline 15.43 & 282.5 & 0.05 & 0.00 \\
\hline 15.67 & 286.6 & 0.05 & 0.00 \\
\hline 16.01 & 292.3 & 0.05 & 0.00 \\
\hline 16.31 & 297.4 & 2.73 & 0.09 \\
\hline 16.53 & 301.2 & 0.04 & 0.00 \\
\hline 16.59 & 302.7 & 0.00 & 0.00 \\
\hline 16.89 & 309.9 & 0.07 & 0.00 \\
\hline 17.18 & 316.9 & 0.04 & 0.00 \\
\hline 17.47 & 324.0 & 0.05 & 0.00 \\
\hline 17.77 & 331.2 & 0.07 & 0.00 \\
\hline 17.98 & 336.3 & 1.87 & 0.03 \\
\hline 18.05 & 338.0 & 15.00 & 0.33 \\
\hline 18.34 & 344.7 & 0.07 & 0.00 \\
\hline 18.63 & 349.4 & 0.29 & 0.01 \\
\hline 18.92 & 354.1 & 0.77 & 0.03 \\
\hline 19.22 & 358.9 & 0.09 & 0.00 \\
\hline 19.44 & 362.3 & 0.06 & 0.00 \\
\hline 19.55 & 363.5 & 0.00 & 0.00 \\
\hline 19.80 & 366.2 & 0.07 & 0.00 \\
\hline 20.07 & 369.1 & 0.06 & 0.00 \\
\hline 20.34 & 372.0 & 0.04 & 0.00 \\
\hline 20.56 & 379.8 & 0.17 & 0.00 \\
\hline 20.80 & 388.2 & 0.06 & 0.00 \\
\hline 21.05 & 397.0 & 0.06 & 0.00 \\
\hline 21.29 & 401.8 & 0.09 & 0.00 \\
\hline 21.56 & 407.1 & 2.28 & 0.05 \\
\hline 21.87 & 413.2 & 25.80 & 0.81 \\
\hline 22.14 & 418.5 & 13.02 & 0.49 \\
\hline 22.39 & 423.3 & 18.20 & 0.43 \\
\hline 22.42 & 423.9 & 4.48 & 0.12 \\
\hline
\end{tabular}

\begin{tabular}{|c|c|c|c|}
\hline $\begin{array}{l}\text { Composite } \\
\text { depth } \\
\text { (m) }\end{array}$ & $\begin{array}{l}\text { Age } \\
\text { (ka) }\end{array}$ & $\begin{array}{c}\mathrm{CaCO}_{3} \\
\text { (wt\%) }\end{array}$ & $\begin{array}{c}\text { MAR } \\
\mathrm{CaCO}_{3} \\
\left(\mathrm{~g} / \mathrm{cm}^{2} \mathrm{k} . \mathrm{y} .\right)\end{array}$ \\
\hline 44.94 & 908.1 & 1.72 & 0.03 \\
\hline 45.18 & 912.8 & 0.09 & 0.00 \\
\hline 45.24 & 914.0 & 0.00 & 0.00 \\
\hline 45.54 & 919.9 & 0.32 & 0.01 \\
\hline 45.84 & 925.8 & 0.06 & 0.00 \\
\hline 46.15 & 931.7 & 0.09 & 0.00 \\
\hline 46.44 & 937.2 & 27.44 & 0.94 \\
\hline 46.68 & 941.8 & 0.13 & 0.00 \\
\hline 46.74 & 942.9 & 0.50 & 0.03 \\
\hline 47.33 & 950.7 & 0.35 & 0.01 \\
\hline 47.65 & 954.4 & 0.22 & 0.01 \\
\hline 47.94 & 958.8 & 0.08 & 0.00 \\
\hline 48.18 & 964.8 & 0.09 & 0.00 \\
\hline 48.24 & 966.4 & 0.00 & 0.00 \\
\hline 48.54 & 973.9 & 0.10 & 0.00 \\
\hline 48.83 & 981.7 & 0.28 & 0.00 \\
\hline 49.25 & 994.1 & 0.06 & 0.00 \\
\hline 49.38 & 997.9 & 0.15 & 0.00 \\
\hline 49.62 & 1003.8 & 2.56 & 0.06 \\
\hline 49.91 & 1010.3 & 0.07 & 0.00 \\
\hline 50.21 & 1017.0 & 0.05 & 0.00 \\
\hline 50.45 & 1022.3 & 0.51 & 0.01 \\
\hline 50.74 & 1028.8 & 0.11 & 0.00 \\
\hline 50.99 & 1034.4 & 0.08 & 0.00 \\
\hline 51.28 & 1040.9 & 0.03 & 0.00 \\
\hline 51.52 & 1046.2 & 0.15 & 0.00 \\
\hline 51.81 & 1051.6 & 0.09 & 0.00 \\
\hline 52.03 & 1054.5 & 0.06 & 0.00 \\
\hline 52.47 & 1060.2 & 0.08 & 0.00 \\
\hline 52.75 & 1064.8 & 0.05 & 0.00 \\
\hline 52.98 & 1068.8 & 0.06 & 0.00 \\
\hline 53.07 & 1070.4 & 5.40 & 0.17 \\
\hline 53.33 & 1075.0 & 29.70 & 1.05 \\
\hline 53.61 & 1080.0 & 28.97 & 0.75 \\
\hline 54.19 & 1096.0 & 0.15 & 0.00 \\
\hline 54.42 & 1102.8 & 0.09 & 0.00 \\
\hline 54.51 & 1105.2 & 0.00 & 0.00 \\
\hline 55.05 & 1118.6 & 11.31 & 0.23 \\
\hline 55.36 & 1126.3 & 10.12 & 0.21 \\
\hline 55.63 & 1133.0 & 0.35 & 0.01 \\
\hline 55.86 & 1140.9 & 0.22 & 0.00 \\
\hline 55.95 & 1144.8 & 0.00 & 0.00 \\
\hline 56.21 & 1156.3 & 0.11 & 0.00 \\
\hline 56.49 & 1168.7 & 0.22 & 0.00 \\
\hline 56.80 & 1180.2 & 5.40 & 0.12 \\
\hline 57.08 & 1186.4 & 0.07 & 0.00 \\
\hline 57.31 & 1191.5 & 0.10 & 0.00 \\
\hline 57.36 & 1192.6 & 0.00 & 0.00 \\
\hline 57.65 & 1198.9 & 1.72 & 0.04 \\
\hline 57.93 & 1205.1 & 6.46 & 0.17 \\
\hline 58.23 & 1210.4 & 13.28 & 0.44 \\
\hline 58.52 & 1215.3 & 12.26 & 0.40 \\
\hline 58.75 & 1219.3 & 2.00 & 0.06 \\
\hline 58.81 & 1220.3 & 0.00 & 0.00 \\
\hline 59.09 & 1225.4 & 0.19 & 0.00 \\
\hline 59.37 & 1231.9 & 0.08 & 0.00 \\
\hline 59.67 & 1238.9 & 0.05 & 0.00 \\
\hline 59.79 & 1241.7 & 0.09 & 0.00 \\
\hline 60.19 & 1251.0 & 0.18 & 0.00 \\
\hline 60.26 & 1252.6 & 0.00 & 0.00 \\
\hline 60.53 & 1257.8 & 0.04 & 0.00 \\
\hline 60.70 & 1260.8 & 0.10 & 0.00 \\
\hline 60.91 & 1264.6 & 2.58 & 0.06 \\
\hline 61.09 & 1267.8 & 9.13 & 0.23 \\
\hline 61.23 & 1270.3 & 11.33 & 0.26 \\
\hline 61.43 & 1274.7 & 1.14 & 0.02 \\
\hline 61.62 & 1281.0 & 0.59 & 0.01 \\
\hline 61.88 & 1289.7 & 0.17 & 0.00 \\
\hline 62.13 & 1298.0 & 0.11 & 0.00 \\
\hline 62.36 & 1305.7 & 0.06 & 0.00 \\
\hline 62.41 & 1307.3 & 0.00 & 0.00 \\
\hline 62.71 & 1315.2 & 0.04 & 0.00 \\
\hline 63.00 & 1322.8 & 0.05 & 0.00 \\
\hline 63.31 & 1331.0 & 0.05 & 0.00 \\
\hline 63.59 & 1338.1 & 0.10 & 0.00 \\
\hline 63.82 & 1343.3 & 0.11 & 0.00 \\
\hline 64.14 & 1350.6 & 0.06 & 0.00 \\
\hline 64.46 & 1357.8 & 0.07 & 0.00 \\
\hline 65.04 & 1385.6 & 0.11 & 0.00 \\
\hline 65.27 & 1392.8 & 0.08 & 0.00 \\
\hline 65.34 & 1394.5 & 0.00 & 0.00 \\
\hline 65.62 & 1401.2 & 11.24 & 0.28 \\
\hline 65.91 & 1408.1 & 24.69 & 0.62 \\
\hline 66.20 & 1415.1 & 0.12 & 0.00 \\
\hline 66.49 & 1422.0 & 0.11 & 0.00 \\
\hline 66.73 & 1429.2 & 0.06 & 0.00 \\
\hline 67.08 & 1441.5 & 16.63 & 0.30 \\
\hline 67.37 & 1452.4 & 0.39 & 0.01 \\
\hline
\end{tabular}


Table 1 (continued).

\begin{tabular}{|c|c|c|c|}
\hline $\begin{array}{l}\text { Composite } \\
\text { depth } \\
\text { (m) }\end{array}$ & $\begin{array}{l}\text { Age } \\
\text { (ka) }\end{array}$ & $\begin{array}{l}\mathrm{CaCO}_{3} \\
(\mathrm{wt} \%)\end{array}$ & $\begin{array}{c}\text { MAR } \\
\mathrm{CaCO}_{3} \\
\left(\mathrm{~g} / \mathrm{cm}^{2} \mathrm{k} . \mathrm{y} .\right)\end{array}$ \\
\hline 67.66 & 1460.3 & 0.09 & 0.00 \\
\hline 67.95 & 1468.6 & 0.20 & 0.00 \\
\hline 68.18 & 1475.4 & 2.17 & 0.04 \\
\hline 68.25 & 1477.5 & 29.30 & 0.48 \\
\hline 68.53 & 1487.7 & 36.33 & 0.57 \\
\hline 68.82 & 1500.5 & 42.26 & 0.80 \\
\hline 69.11 & 1509.3 & 0.36 & 0.01 \\
\hline 69.41 & 1516.8 & 0.16 & 0.00 \\
\hline 69.64 & 1522.5 & 0.05 & 0.00 \\
\hline 69.99 & 1531.3 & 0.07 & 0.00 \\
\hline 70.41 & 1540.7 & 0.04 & 0.00 \\
\hline 70.65 & 1545.1 & 0.10 & 0.00 \\
\hline 70.84 & 1548.5 & 12.42 & 0.34 \\
\hline 71.02 & 1551.7 & 11.65 & 0.34 \\
\hline 71.22 & 1555.3 & 27.23 & 0.98 \\
\hline 71.41 & 1558.8 & 31.74 & 1.26 \\
\hline 71.79 & 1565.6 & 0.13 & 0.00 \\
\hline 71.98 & 1572.9 & 0.13 & 0.00 \\
\hline 72.17 & 1580.7 & 0.05 & 0.00 \\
\hline 72.36 & 1587.1 & 0.09 & 0.00 \\
\hline 72.45 & 1588.6 & 8.08 & 0.30 \\
\hline 72.51 & 1589.7 & 17.70 & 0.64 \\
\hline 72.79 & 1594.5 & 0.41 & 0.02 \\
\hline 73.08 & 1599.6 & 0.14 & 0.00 \\
\hline 73.37 & 1604.7 & 0.08 & 0.00 \\
\hline 73.66 & 1609.0 & 0.13 & 0.01 \\
\hline 73.89 & 1612.4 & 0.20 & 0.01 \\
\hline 73.96 & 1613.4 & 0.00 & 0.00 \\
\hline 74.24 & 1617.5 & 0.08 & 0.00 \\
\hline 74.53 & 1621.8 & 0.11 & 0.00 \\
\hline 74.84 & 1627.5 & 0.05 & 0.00 \\
\hline 75.11 & 1635.0 & 0.06 & 0.00 \\
\hline 75.34 & 1641.4 & 0.05 & 0.00 \\
\hline 75.40 & 1643.1 & 0.00 & 0.00 \\
\hline 75.69 & 1649.5 & 0.16 & 0.00 \\
\hline 75.98 & 1655.4 & 2.38 & 0.07 \\
\hline 76.28 & 1661.5 & 0.04 & 0.00 \\
\hline 76.55 & 1667.1 & 0.03 & 0.00 \\
\hline 76.79 & 1672.0 & 0.07 & 0.00 \\
\hline 76.84 & 1673.0 & 0.00 & 0.00 \\
\hline 77.13 & 1677.6 & 0.14 & 0.01 \\
\hline 77.31 & 1680.3 & 0.29 & 0.01 \\
\hline 77.42 & 1682.0 & 0.20 & 0.01 \\
\hline 77.60 & 1684.7 & 9.27 & 0.40 \\
\hline 77.73 & 1686.6 & 16.91 & 0.74 \\
\hline 78.00 & 1690.7 & 6.00 & 0.32 \\
\hline 78.23 & 1694.1 & 0.16 & 0.01 \\
\hline 78.29 & 1695.1 & 0.00 & 0.00 \\
\hline 78.37 & 1696.3 & 0.00 & 0.00 \\
\hline 78.58 & 1702.3 & 0.03 & 0.00 \\
\hline 78.87 & 1710.1 & 0.13 & 0.00 \\
\hline 79.12 & 1714.4 & 0.15 & 0.01 \\
\hline 79.45 & 1721.2 & 0.02 & 0.00 \\
\hline 79.68 & 1727.5 & 0.09 & 0.00 \\
\hline 79.73 & 1728.6 & 0.00 & 0.00 \\
\hline 79.81 & 1730.0 & 0.06 & 0.00 \\
\hline 80.02 & 1733.9 & 0.10 & 0.00 \\
\hline 80.29 & 1738.9 & 0.09 & 0.00 \\
\hline 80.39 & 1740.7 & 0.17 & 0.01 \\
\hline 80.48 & 1742.3 & 0.21 & 0.01 \\
\hline 80.57 & 1744.0 & 0.35 & 0.01 \\
\hline 80.67 & 1745.8 & 0.21 & 0.01 \\
\hline 80.76 & 1747.5 & 0.15 & 0.01 \\
\hline 80.86 & 1749.3 & 0.10 & 0.00 \\
\hline 80.97 & 1751.4 & 0.09 & 0.00 \\
\hline 81.04 & 1752.6 & 0.16 & 0.01 \\
\hline 81.14 & 1754.5 & 0.13 & 0.00 \\
\hline 81.23 & 1756.1 & 0.09 & 0.00 \\
\hline 81.37 & 1758.7 & 0.10 & 0.00 \\
\hline 81.47 & 1762.1 & 0.15 & 0.00 \\
\hline 81.56 & 1768.4 & 1.55 & 0.01 \\
\hline 81.66 & 1775.3 & 5.40 & 0.05 \\
\hline 81.75 & 1781.6 & 1.57 & 0.01 \\
\hline 81.85 & 1788.6 & 0.94 & 0.01 \\
\hline 81.94 & 1794.8 & 0.68 & 0.01 \\
\hline 82.03 & 1800.3 & 0.52 & 0.01 \\
\hline 82.07 & 1802.1 & 0.08 & 0.00 \\
\hline 82.16 & 1806.2 & 0.20 & 0.00 \\
\hline 82.24 & 1809.8 & 0.09 & 0.00 \\
\hline 82.45 & 1825.9 & 0.08 & 0.00 \\
\hline 82.51 & 1831.1 & 0.03 & 0.00 \\
\hline 82.68 & 1843.6 & 0.07 & 0.00 \\
\hline 82.74 & 1848.5 & 0.00 & 0.00 \\
\hline 82.82 & 1855.4 & 0.05 & 0.00 \\
\hline 83.03 & 1873.6 & 0.10 & 0.00 \\
\hline 83.21 & 1889.3 & 0.00 & 0.00 \\
\hline 83.32 & 1898.8 & 0.14 & 0.00 \\
\hline 83.51 & 1911.8 & 0.07 & 0.00 \\
\hline
\end{tabular}

\begin{tabular}{|c|c|c|c|}
\hline $\begin{array}{l}\text { Composite } \\
\text { depth } \\
\text { (m) }\end{array}$ & $\begin{array}{l}\text { Age } \\
\text { (ka) }\end{array}$ & $\begin{array}{c}\mathrm{CaCO}_{3} \\
\text { (wt\%) }\end{array}$ & $\begin{array}{c}\mathrm{MAR} \\
\mathrm{CaCO}_{3} \\
\left(\mathrm{~g} / \mathrm{cm}^{2} \mathrm{k} . \mathrm{y} .\right)\end{array}$ \\
\hline 83.61 & 1917.8 & 0.06 & 0.00 \\
\hline 83.69 & 1922.5 & 0.04 & 0.00 \\
\hline 83.90 & 1934.8 & 0.07 & 0.00 \\
\hline 84.14 & 1947.7 & 0.02 & 0.00 \\
\hline 84.20 & 1949.0 & 0.00 & 0.00 \\
\hline 84.49 & 1955.5 & 0.07 & 0.00 \\
\hline 84.66 & 1959.3 & 0.04 & 0.00 \\
\hline 84.78 & 1962.0 & 0.10 & 0.00 \\
\hline 84.91 & 1964.9 & 0.10 & 0.00 \\
\hline 85.07 & 1968.5 & 2.28 & 0.08 \\
\hline 85.14 & 1970.1 & 2.51 & 0.08 \\
\hline 85.34 & 1974.6 & 0.09 & 0.00 \\
\hline 85.42 & 1976.7 & 0.02 & 0.00 \\
\hline 85.59 & 1981.4 & 2.09 & 0.05 \\
\hline 85.65 & 1983.1 & 0.00 & 0.00 \\
\hline 85.73 & 1985.3 & 0.08 & 0.00 \\
\hline 85.94 & 1991.1 & 0.19 & 0.00 \\
\hline 86.09 & 1995.3 & 0.36 & 0.01 \\
\hline 86.23 & 1999.2 & 13.55 & 0.29 \\
\hline 86.41 & 2004.2 & 0.50 & 0.01 \\
\hline 86.52 & 2007.3 & 0.07 & 0.00 \\
\hline 86.60 & 2009.5 & 0.06 & 0.00 \\
\hline 86.81 & 2017.2 & 0.32 & 0.01 \\
\hline 86.88 & 2020.2 & 0.20 & 0.00 \\
\hline 87.02 & 2026.2 & 0.00 & 0.00 \\
\hline 87.09 & 2029.2 & 0.10 & 0.00 \\
\hline 87.18 & 2033.1 & 0.08 & 0.00 \\
\hline 87.40 & 2042.5 & 0.12 & 0.00 \\
\hline 87.57 & 2049.9 & 0.08 & 0.00 \\
\hline 87.69 & 2054.2 & 0.10 & 0.00 \\
\hline 87.83 & 2058.6 & 0.04 & 0.00 \\
\hline 87.98 & 2063.2 & 0.06 & 0.00 \\
\hline 88.09 & 2066.7 & 0.38 & 0.01 \\
\hline 88.13 & 2067.9 & 1.96 & 0.04 \\
\hline 88.33 & 2074.2 & 9.63 & 0.22 \\
\hline 88.46 & 2078.2 & 0.10 & 0.00 \\
\hline 88.60 & 2082.6 & 0.12 & 0.00 \\
\hline 88.62 & 2083.2 & 0.00 & 0.00 \\
\hline 88.85 & 2092.8 & 0.04 & 0.00 \\
\hline 89.03 & 2101.6 & 0.06 & 0.00 \\
\hline 89.14 & 2107.0 & 0.05 & 0.00 \\
\hline 89.30 & 2114.8 & 0.05 & 0.00 \\
\hline 89.43 & 2121.2 & 1.30 & 0.02 \\
\hline 89.52 & 2125.6 & 23.90 & 0.36 \\
\hline 89.72 & 2134.5 & 15.45 & 0.23 \\
\hline 89.81 & 2138.1 & 0.96 & 0.02 \\
\hline 89.94 & 2143.4 & 0.08 & 0.00 \\
\hline 89.97 & 2144.6 & 0.00 & 0.00 \\
\hline 90.08 & 2149.1 & 0.06 & 0.00 \\
\hline 90.31 & 2158.4 & 0.75 & 0.01 \\
\hline 90.77 & 2173.5 & 0.06 & 0.00 \\
\hline 90.97 & 2176.9 & 0.08 & 0.00 \\
\hline 91.17 & 2180.3 & 0.10 & 0.00 \\
\hline 91.37 & 2183.7 & 5.27 & 0.17 \\
\hline 91.57 & 2187.1 & 0.03 & 0.00 \\
\hline 91.77 & 2190.5 & 0.08 & 0.00 \\
\hline 91.97 & 2194.2 & 0.21 & 0.01 \\
\hline 92.17 & 2197.8 & 0.10 & 0.00 \\
\hline 92.57 & 2205.0 & 0.12 & 0.00 \\
\hline 92.87 & 2210.4 & 5.51 & 0.18 \\
\hline 93.09 & 2214.4 & 9.90 & 0.32 \\
\hline 93.14 & 2215.3 & 0.70 & 0.03 \\
\hline 93.22 & 2216.6 & 0.06 & 0.00 \\
\hline 93.44 & 2220.0 & 1.45 & 0.05 \\
\hline 93.61 & 2222.6 & 0.13 & 0.00 \\
\hline 93.72 & 2224.2 & 0.07 & 0.00 \\
\hline 94.01 & 2228.7 & 0.10 & 0.00 \\
\hline 94.30 & 2233.1 & 0.45 & 0.01 \\
\hline 94.49 & 2236.0 & 1.21 & 0.04 \\
\hline 94.67 & 2238.7 & 0.27 & 0.01 \\
\hline 94.88 & 2241.9 & 3.35 & 0.13 \\
\hline 95.05 & 2244.5 & 0.00 & 0.00 \\
\hline 95.17 & 2246.4 & 0.13 & 0.01 \\
\hline 95.46 & 2250.8 & 0.13 & 0.00 \\
\hline 95.74 & 2259.0 & 0.05 & 0.00 \\
\hline 95.98 & 2267.5 & 0.10 & 0.00 \\
\hline 96.02 & 2268.9 & 0.00 & 0.00 \\
\hline 96.11 & 2272.0 & 0.14 & 0.00 \\
\hline 96.32 & 2279.4 & 1.74 & 0.02 \\
\hline 96.49 & 2285.4 & 19.47 & 0.34 \\
\hline 96.61 & 2289.6 & 28.50 & 0.60 \\
\hline 96.90 & 2299.8 & 12.85 & 0.21 \\
\hline 97.19 & 2310.0 & 3.38 & 0.04 \\
\hline 97.42 & 2323.2 & 4.46 & 0.04 \\
\hline 97.55 & 2331.2 & 0.39 & 0.00 \\
\hline 97.76 & 2344.0 & 0.09 & 0.00 \\
\hline 97.94 & 2355.0 & 5.30 & 0.05 \\
\hline 98.05 & 2361.8 & 9.63 & 0.11 \\
\hline
\end{tabular}

\begin{tabular}{|c|c|c|c|}
\hline $\begin{array}{c}\text { Composite } \\
\text { depth } \\
\text { (m) }\end{array}$ & $\begin{array}{l}\text { Age } \\
\text { (ka) }\end{array}$ & $\begin{array}{c}\mathrm{CaCO}_{3} \\
(\mathrm{wt} \%)\end{array}$ & $\begin{array}{c}\text { MAR } \\
\mathrm{CaCO}_{3} \\
\left(\mathrm{~g} / \mathrm{cm}^{2} \mathrm{k} \cdot \mathrm{y} .\right)\end{array}$ \\
\hline 98.36 & 2376.0 & 22.88 & 0.49 \\
\hline 98.63 & 2380.8 & 0.06 & 0.00 \\
\hline 98.85 & 2384.7 & 0.00 & 0.00 \\
\hline 98.86 & 2384.9 & 0.06 & 0.00 \\
\hline 99.00 & 2387.3 & 0.00 & 0.00 \\
\hline 99.21 & 2391.1 & 0.06 & 0.00 \\
\hline 99.38 & 2394.1 & 0.00 & 0.00 \\
\hline 99.50 & 2396.2 & 0.10 & 0.00 \\
\hline 99.78 & 2401.2 & 0.07 & 0.00 \\
\hline 100.07 & 2406.4 & 0.22 & 0.01 \\
\hline 100.30 & 2410.5 & 0.09 & 0.00 \\
\hline 100.44 & 2413.6 & 0.06 & 0.00 \\
\hline 100.65 & 2418.6 & 0.10 & 0.00 \\
\hline 100.84 & 2423.1 & 0.00 & 0.00 \\
\hline 101.12 & 2430.3 & 7.64 & 0.16 \\
\hline 101.31 & 2438.4 & 0.16 & 0.00 \\
\hline 101.50 & 2446.5 & 0.10 & 0.00 \\
\hline 101.70 & 2454.8 & 0.07 & 0.00 \\
\hline 101.88 & 2462.3 & 0.09 & 0.00 \\
\hline 102.03 & 2468.6 & 0.07 & 0.00 \\
\hline 102.22 & 2476.5 & 0.06 & 0.00 \\
\hline 102.41 & 2484.4 & 4.44 & 0.06 \\
\hline 102.50 & 2488.2 & 5.32 & 0.07 \\
\hline 102.75 & 2500.9 & 3.60 & 0.04 \\
\hline 103.05 & 2516.4 & 0.10 & 0.00 \\
\hline 103.28 & 2528.3 & 0.05 & 0.00 \\
\hline 103.34 & 2531.4 & 0.00 & 0.00 \\
\hline 103.63 & 2541.8 & 0.06 & 0.00 \\
\hline 103.92 & 2551.3 & 0.10 & 0.00 \\
\hline 104.21 & 2560.8 & 0.20 & 0.00 \\
\hline 104.50 & 2570.2 & 0.04 & 0.00 \\
\hline 104.73 & 2577.7 & 0.39 & 0.01 \\
\hline 104.87 & 2581.9 & 0.00 & 0.00 \\
\hline 105.08 & 2587.5 & 0.07 & 0.00 \\
\hline 105.26 & 2592.9 & 0.00 & 0.00 \\
\hline 105.38 & 2597.4 & 0.05 & 0.00 \\
\hline 105.67 & 2608.2 & 0.05 & 0.00 \\
\hline 105.96 & 2619.0 & 0.06 & 0.00 \\
\hline 106.19 & 2626.2 & 0.06 & 0.00 \\
\hline 106.23 & 2626.9 & 0.00 & 0.00 \\
\hline 106.54 & 2632.3 & 0.05 & 0.00 \\
\hline 106.83 & 2637.3 & 0.15 & 0.01 \\
\hline 107.12 & 2642.4 & 0.08 & 0.00 \\
\hline 107.41 & 2647.4 & 2.08 & 0.07 \\
\hline 107.64 & 2652.7 & 4.54 & 0.10 \\
\hline 108.01 & 2665.2 & 3.58 & 0.07 \\
\hline 108.29 & 2673.5 & 5.68 & 0.14 \\
\hline 108.58 & 2679.0 & 3.21 & 0.07 \\
\hline 108.87 & 2684.4 & 0.50 & 0.01 \\
\hline 109.10 & 2688.8 & 0.14 & 0.00 \\
\hline 109.14 & 2689.5 & 0.00 & 0.00 \\
\hline 109.45 & 2695.4 & 3.51 & 0.11 \\
\hline 109.74 & 2701.0 & 9.00 & 0.27 \\
\hline 110.03 & 2706.7 & 1.01 & 0.03 \\
\hline 110.32 & 2712.3 & 0.12 & 0.00 \\
\hline 110.56 & 2717.0 & 0.31 & 0.01 \\
\hline 110.91 & 2723.8 & 3.09 & 0.10 \\
\hline 111.20 & 2729.2 & 0.28 & 0.01 \\
\hline 111.76 & 2734.3 & 0.43 & 0.02 \\
\hline 112.06 & 2737.0 & 0.14 & 0.01 \\
\hline 112.35 & 2739.7 & 1.65 & 0.07 \\
\hline 112.64 & 2742.3 & 0.10 & 0.00 \\
\hline 112.95 & 2745.2 & 0.04 & 0.00 \\
\hline 113.24 & 2747.8 & 0.08 & 0.00 \\
\hline 113.46 & 2749.8 & 0.74 & 0.03 \\
\hline 113.52 & 2750.4 & 0.00 & 0.00 \\
\hline 113.80 & 2752.9 & 0.21 & 0.01 \\
\hline 114.08 & 2756.0 & 0.24 & 0.01 \\
\hline 114.37 & 2759.1 & 0.10 & 0.00 \\
\hline 114.66 & 2762.3 & 0.09 & 0.00 \\
\hline 114.89 & 2764.8 & 0.24 & 0.01 \\
\hline 115.23 & 2768.5 & 0.11 & 0.00 \\
\hline 115.50 & 2771.5 & 0.09 & 0.00 \\
\hline 115.79 & 2774.6 & 0.04 & 0.00 \\
\hline 116.08 & 2777.8 & 0.23 & 0.01 \\
\hline 116.31 & 2780.3 & 0.27 & 0.01 \\
\hline 116.37 & 2781.0 & 0.00 & 0.00 \\
\hline 116.65 & 2784.0 & 0.04 & 0.00 \\
\hline 116.92 & 2786.4 & 0.05 & 0.00 \\
\hline 117.22 & 2788.6 & 0.04 & 0.00 \\
\hline 117.50 & 2790.7 & 0.63 & 0.03 \\
\hline 117.73 & 2792.4 & 0.50 & 0.03 \\
\hline 118.07 & 2795.0 & 0.86 & 0.04 \\
\hline 118.35 & 2797.1 & 1.27 & 0.06 \\
\hline 118.64 & 2799.3 & 0.29 & 0.02 \\
\hline 118.92 & 2801.4 & 0.30 & 0.01 \\
\hline 119.15 & 2803.1 & 0.85 & 0.05 \\
\hline 119.49 & 2805.7 & 4.13 & 0.20 \\
\hline
\end{tabular}


Table 1 (continued).

\begin{tabular}{|c|c|c|c|}
\hline $\begin{array}{l}\text { Composite } \\
\text { depth } \\
\text { (m) }\end{array}$ & $\begin{array}{l}\text { Age } \\
\text { (ka) }\end{array}$ & $\begin{array}{r}\mathrm{CaCO}_{3} \\
\text { (wt } \%)\end{array}$ & $\begin{array}{c}\text { MAR } \\
\mathrm{CaCO}_{3} \\
\left(\mathrm{~g} / \mathrm{cm}^{2} \mathrm{k} . \mathrm{y} .\right)\end{array}$ \\
\hline 119.77 & 2807.8 & 0.13 & 0.01 \\
\hline 120.06 & 2809.9 & 0.05 & 0.00 \\
\hline 120.62 & 2814.6 & 0.05 & 0.00 \\
\hline 120.91 & 2817.0 & 0.29 & 0.01 \\
\hline 121.19 & 2819.3 & 0.08 & 0.00 \\
\hline 121.57 & 2822.5 & 0.04 & 0.00 \\
\hline 121.76 & 2824.1 & 0.83 & 0.03 \\
\hline 122.05 & 2826.5 & 0.06 & 0.00 \\
\hline 122.33 & 2828.8 & 5.55 & 0.24 \\
\hline 122.62 & 2831.3 & 5.45 & 0.26 \\
\hline 122.90 & 2833.6 & 8.37 & 0.40 \\
\hline 123.19 & 2836.0 & 7.28 & 0.35 \\
\hline 123.26 & 2836.6 & 8.10 & 0.37 \\
\hline 123.52 & 2838.8 & 8.96 & 0.42 \\
\hline 123.83 & 2841.3 & 11.18 & 0.56 \\
\hline 124.12 & 2843.8 & 1.75 & 0.07 \\
\hline 124.41 & 2846.4 & 0.33 & 0.01 \\
\hline 124.64 & 2848.5 & 0.25 & 0.01 \\
\hline 125.00 & 2851.8 & 0.06 & 0.00 \\
\hline 125.29 & 2854.5 & 0.24 & 0.01 \\
\hline 125.58 & 2857.1 & 0.06 & 0.00 \\
\hline 125.87 & 2859.8 & 0.08 & 0.00 \\
\hline 126.10 & 2861.9 & 0.05 & 0.00 \\
\hline 126.21 & 2862.9 & 0.00 & 0.00 \\
\hline 126.45 & 2865.1 & 0.07 & 0.00 \\
\hline 126.74 & 2867.8 & 0.08 & 0.00 \\
\hline 127.03 & 2870.5 & 2.92 & 0.13 \\
\hline 127.33 & 2873.2 & 12.32 & 0.55 \\
\hline 127.56 & 2875.3 & 13.53 & 0.74 \\
\hline 127.91 & 2878.5 & 12.67 & 0.86 \\
\hline 128.20 & 2880.8 & 8.76 & 0.52 \\
\hline 128.49 & 2883.1 & 5.20 & 0.28 \\
\hline 128.78 & 2885.3 & 0.62 & 0.03 \\
\hline 129.02 & 2887.2 & 0.06 & 0.00 \\
\hline 129.06 & 2887.6 & 0.00 & 0.00 \\
\hline 129.37 & 2890.0 & 0.06 & 0.00 \\
\hline 129.66 & 2892.3 & 0.07 & 0.00 \\
\hline 129.95 & 2894.6 & 4.04 & 0.21 \\
\hline 130.24 & 2896.9 & 5.30 & 0.29 \\
\hline 130.47 & 2898.7 & 4.94 & 0.25 \\
\hline 130.82 & 2901.5 & 8.54 & 0.38 \\
\hline 131.11 & 2903.7 & 14.46 & 0.79 \\
\hline 131.39 & 2905.7 & 19.05 & 1.50 \\
\hline 131.68 & 2907.5 & 13.47 & 1.08 \\
\hline 131.97 & 2909.2 & 8.15 & 0.54 \\
\hline 132.55 & 2912.7 & 1.97 & 0.14 \\
\hline 132.84 & 2914.4 & 0.08 & 0.01 \\
\hline 133.13 & 2916.2 & 2.86 & 0.19 \\
\hline 133.41 & 2917.9 & 1.65 & 0.11 \\
\hline 133.83 & 2920.4 & 1.37 & 0.11 \\
\hline 134.28 & 2923.1 & 3.73 & 0.27 \\
\hline 134.49 & 2924.3 & 8.01 & 0.49 \\
\hline 134.79 & 2926.1 & 12.82 & 1.01 \\
\hline 135.02 & 2927.5 & 17.65 & 1.25 \\
\hline 135.38 & 2929.7 & 17.35 & 1.38 \\
\hline 135.67 & 2931.4 & 10.27 & 0.68 \\
\hline 135.97 & 2933.2 & 5.91 & 0.35 \\
\hline 136.27 & 2935.0 & 5.11 & 0.35 \\
\hline 136.51 & 2936.5 & 6.82 & 0.42 \\
\hline 136.57 & 2936.8 & 5.80 & 0.41 \\
\hline 136.87 & 2938.5 & 5.29 & 0.31 \\
\hline 137.15 & 2940.1 & 4.20 & 0.23 \\
\hline 137.46 & 2941.8 & 2.53 & 0.16 \\
\hline 137.76 & 2943.5 & 0.88 & 0.06 \\
\hline 137.99 & 2944.8 & 0.27 & 0.02 \\
\hline 138.35 & 2946.8 & 0.53 & 0.04 \\
\hline 138.64 & 2948.4 & 0.44 & 0.04 \\
\hline 138.95 & 2950.2 & 0.25 & 0.02 \\
\hline 139.24 & 2951.8 & 0.13 & 0.01 \\
\hline 139.48 & 2953.1 & 0.34 & 0.03 \\
\hline 139.84 & 2955.2 & 0.23 & 0.01 \\
\hline 140.12 & 2956.7 & 0.11 & 0.01 \\
\hline 140.43 & 2958.5 & 0.75 & 0.05 \\
\hline 140.74 & 2960.2 & 0.20 & 0.01 \\
\hline 140.97 & 2961.5 & 7.64 & 0.58 \\
\hline 141.32 & 2963.5 & 21.82 & 1.94 \\
\hline 141.60 & 2965.0 & 24.45 & 2.76 \\
\hline 141.90 & 2966.7 & 38.06 & 4.18 \\
\hline 142.19 & 2968.3 & 34.95 & 4.05 \\
\hline 142.47 & 2969.9 & 25.20 & 2.54 \\
\hline 142.76 & 2971.5 & 30.18 & 2.99 \\
\hline 143.04 & 2973.1 & 25.49 & 2.27 \\
\hline 143.33 & 2974.7 & 18.02 & 1.60 \\
\hline 143.62 & 2976.4 & 12.67 & 1.05 \\
\hline 143.87 & 2977.8 & 9.70 & 0.71 \\
\hline 144.15 & 2979.3 & 7.59 & 0.59 \\
\hline 144.21 & 2979.7 & 7.20 & 0.56 \\
\hline 144.51 & 2981.4 & 4.55 & 0.26 \\
\hline
\end{tabular}

\begin{tabular}{|c|c|c|c|}
\hline $\begin{array}{l}\text { Composite } \\
\text { depth } \\
\text { (m) }\end{array}$ & $\begin{array}{l}\text { Age } \\
\text { (ka) }\end{array}$ & $\begin{array}{c}\mathrm{CaCO}_{3} \\
\text { (wt } \%)\end{array}$ & $\begin{array}{c}\mathrm{MAR} \\
\mathrm{CaCO}_{3} \\
\left(\mathrm{~g} / \mathrm{cm}^{2} \mathrm{k} . \mathrm{y} .\right)\end{array}$ \\
\hline 144.81 & 2983.3 & 7.72 & 0.47 \\
\hline 145.11 & 2985.2 & 4.23 & 0.25 \\
\hline 145.41 & 2987.1 & 6.93 & 0.43 \\
\hline 145.65 & 2988.6 & 17.16 & 1.32 \\
\hline 146.01 & 2990.9 & 28.82 & 2.30 \\
\hline 146.31 & 2992.8 & 25.50 & 1.99 \\
\hline 146.61 & 2994.7 & 26.69 & 2.19 \\
\hline 146.91 & 2996.6 & 18.33 & 1.24 \\
\hline 147.15 & 2998.2 & 20.60 & 1.45 \\
\hline 147.20 & 2998.5 & 21.50 & 1.64 \\
\hline 147.51 & 3000.5 & 15.48 & 1.01 \\
\hline 147.81 & 3002.4 & 11.46 & 0.72 \\
\hline 148.11 & 3004.3 & 8.37 & 0.57 \\
\hline 148.41 & 3006.2 & 5.72 & 0.35 \\
\hline 148.65 & 3007.7 & 6.72 & 0.48 \\
\hline 149.01 & 3009.8 & 6.61 & 0.43 \\
\hline 149.31 & 3011.7 & 7.16 & 0.48 \\
\hline 149.61 & 3013.5 & 5.92 & 0.35 \\
\hline 149.91 & 3015.3 & 7.91 & 0.55 \\
\hline 150.15 & 3016.7 & 9.22 & 0.85 \\
\hline 150.21 & 3017.1 & 9.10 & 0.77 \\
\hline 150.51 & 3018.9 & 5.12 & 0.31 \\
\hline 150.81 & 3020.7 & 2.73 & 0.20 \\
\hline 151.11 & 3022.6 & 2.52 & 0.18 \\
\hline 151.41 & 3024.4 & 0.53 & 0.05 \\
\hline 151.65 & 3025.8 & 2.07 & 0.18 \\
\hline 151.91 & 3027.4 & 1.25 & 0.07 \\
\hline 152.48 & 3030.8 & 0.32 & 0.02 \\
\hline 152.77 & 3032.4 & 0.23 & 0.02 \\
\hline 153.05 & 3034.0 & 0.80 & 0.06 \\
\hline 153.34 & 3035.7 & 4.61 & 0.31 \\
\hline 153.62 & 3037.3 & 8.60 & 0.55 \\
\hline 154.13 & 3040.2 & 7.76 & 0.57 \\
\hline 154.19 & 3040.5 & 8.80 & 0.67 \\
\hline 154.49 & 3042.2 & 4.38 & 0.33 \\
\hline 154.77 & 3043.8 & 5.50 & 0.37 \\
\hline 155.07 & 3045.5 & 2.14 & 0.14 \\
\hline 155.37 & 3047.2 & 0.49 & 0.03 \\
\hline 155.60 & 3048.5 & 0.15 & 0.01 \\
\hline 155.96 & 3050.5 & 0.05 & 0.00 \\
\hline 156.24 & 3052.1 & 0.20 & 0.01 \\
\hline 156.54 & 3053.8 & 0.31 & 0.02 \\
\hline 156.84 & 3055.7 & 1.16 & 0.06 \\
\hline 157.07 & 3057.1 & 1.91 & 0.11 \\
\hline 157.13 & 3057.5 & 3.60 & 0.23 \\
\hline 157.42 & 3059.3 & 3.86 & 0.27 \\
\hline 157.71 & 3061.2 & 1.74 & 0.10 \\
\hline 158.31 & 3064.9 & 3.16 & 0.17 \\
\hline 158.54 & 3066.4 & 5.12 & 0.32 \\
\hline 158.89 & 3068.6 & 4.93 & 0.34 \\
\hline 159.18 & 3070.4 & 14.91 & 1.14 \\
\hline 159.48 & 3072.3 & 11.47 & 0.76 \\
\hline 159.77 & 3074.1 & 18.05 & 1.08 \\
\hline 160.01 & 3076.0 & 17.04 & 0.74 \\
\hline 160.07 & 3076.6 & 20.00 & 0.81 \\
\hline 160.36 & 3079.4 & 28.53 & 1.52 \\
\hline 160.65 & 3082.3 & 25.57 & 1.25 \\
\hline 160.95 & 3085.2 & 30.34 & 1.64 \\
\hline 161.24 & 3088.1 & 22.02 & 1.23 \\
\hline 161.53 & 3091.0 & 22.58 & 1.10 \\
\hline 161.82 & 3093.8 & 23.10 & 1.25 \\
\hline 162.11 & 3096.7 & 21.28 & 1.11 \\
\hline 162.40 & 3099.5 & 22.54 & 1.14 \\
\hline 162.68 & 3102.3 & 24.20 & 1.31 \\
\hline 162.97 & 3105.1 & 21.77 & 0.99 \\
\hline 163.26 & 3108.0 & 21.91 & 0.96 \\
\hline 163.55 & 3110.8 & 17.60 & 0.83 \\
\hline 163.84 & 3113.7 & 18.89 & 0.87 \\
\hline 164.13 & 3116.6 & 17.41 & 0.82 \\
\hline 164.41 & 3119.3 & 14.30 & 0.63 \\
\hline 164.70 & 3122.2 & 15.03 & 0.63 \\
\hline 164.99 & 3125.0 & 11.48 & 0.58 \\
\hline 165.14 & 3126.5 & 10.95 & 0.51 \\
\hline 165.43 & 3129.4 & 12.11 & 0.50 \\
\hline 165.72 & 3132.2 & 12.36 & 0.60 \\
\hline 166.01 & 3135.1 & 12.16 & 0.54 \\
\hline 166.24 & 3137.3 & 6.67 & 0.31 \\
\hline 166.59 & 3140.8 & 4.41 & 0.18 \\
\hline 166.88 & 3143.6 & 1.87 & 0.08 \\
\hline 167.18 & 3146.4 & 2.49 & 0.13 \\
\hline 167.47 & 3148.5 & 1.31 & 0.08 \\
\hline 167.70 & 3150.1 & 1.24 & 0.08 \\
\hline 167.76 & 3150.5 & 1.50 & 0.09 \\
\hline 168.05 & 3152.6 & 7.59 & 0.55 \\
\hline 168.34 & 3154.7 & 26.56 & 1.69 \\
\hline 168.63 & 3156.7 & 19.37 & 1.30 \\
\hline 168.93 & 3158.8 & 24.65 & 1.67 \\
\hline 169.16 & 3160.5 & 22.93 & 1.50 \\
\hline
\end{tabular}

\begin{tabular}{|c|c|c|c|}
\hline $\begin{array}{l}\text { Composite } \\
\text { depth } \\
\text { (m) }\end{array}$ & $\begin{array}{l}\text { Age } \\
\text { (ka) }\end{array}$ & $\begin{array}{c}\mathrm{CaCO}_{3} \\
(\mathrm{wt} \%)\end{array}$ & $\begin{array}{c}\mathrm{MAR} \\
\mathrm{CaCO}_{3} \\
\left(\mathrm{~g} / \mathrm{cm}^{2} \mathrm{k} . \mathrm{y} .\right)\end{array}$ \\
\hline 169.51 & 3163.0 & 12.66 & 0.99 \\
\hline 169.80 & 3165.0 & 20.96 & 1.36 \\
\hline 170.09 & 3167.1 & 14.41 & 0.65 \\
\hline 170.38 & 3170.1 & 18.17 & 0.76 \\
\hline 170.61 & 3172.5 & 20.93 & 0.93 \\
\hline 170.67 & 3173.1 & 18.70 & 0.80 \\
\hline 170.96 & 3176.1 & 12.66 & 0.51 \\
\hline 171.26 & 3179.2 & 10.01 & 0.35 \\
\hline 171.55 & 3182.2 & 6.56 & 0.23 \\
\hline 171.84 & 3185.1 & 7.05 & 0.27 \\
\hline 172.07 & 3187.5 & 6.63 & 0.25 \\
\hline 172.42 & 3191.1 & 14.88 & 0.58 \\
\hline 172.71 & 3194.1 & 20.97 & 0.88 \\
\hline 172.98 & 3196.9 & 21.72 & 1.09 \\
\hline 173.17 & 3198.1 & 22.98 & 1.89 \\
\hline 173.46 & 3199.8 & 18.95 & 1.42 \\
\hline 173.74 & 3201.5 & 17.42 & 1.38 \\
\hline 174.03 & 3203.2 & 19.40 & 1.46 \\
\hline 174.44 & 3205.7 & 16.31 & 1.33 \\
\hline 174.67 & 3207.0 & 19.86 & 1.49 \\
\hline 174.73 & 3207.4 & 19.00 & 1.39 \\
\hline 175.01 & 3209.1 & 16.09 & 1.30 \\
\hline 175.29 & 3210.7 & 12.80 & 0.85 \\
\hline 175.57 & 3212.4 & 14.98 & 1.19 \\
\hline 175.85 & 3214.1 & 15.10 & 1.18 \\
\hline 176.08 & 3215.4 & 8.44 & 0.65 \\
\hline 176.42 & 3217.5 & 6.87 & 0.54 \\
\hline 176.70 & 3219.1 & 9.59 & 0.73 \\
\hline 176.98 & 3220.8 & 9.63 & 0.67 \\
\hline 177.26 & 3222.5 & 9.36 & 0.72 \\
\hline 177.49 & 3223.8 & 7.87 & 0.61 \\
\hline 177.54 & 3224.1 & 10.10 & 0.69 \\
\hline 177.83 & 3225.9 & 13.01 & 1.18 \\
\hline 178.11 & 3227.5 & 15.99 & 1.25 \\
\hline 178.39 & 3229.2 & 18.47 & 1.45 \\
\hline 178.67 & 3230.9 & 26.39 & 2.15 \\
\hline 178.90 & 3232.7 & 28.96 & 2.14 \\
\hline 179.24 & 3235.4 & 22.79 & 1.48 \\
\hline 179.52 & 3237.6 & 8.68 & 0.47 \\
\hline 179.80 & 3239.8 & 5.76 & 0.31 \\
\hline 180.08 & 3242.1 & 6.84 & 0.34 \\
\hline 180.31 & 3243.9 & 11.24 & 0.59 \\
\hline 180.37 & 3244.4 & 13.20 & 0.72 \\
\hline 180.65 & 3246.6 & 21.21 & 1.28 \\
\hline 180.93 & 3248.8 & 26.56 & 1.73 \\
\hline 181.21 & 3251.1 & 36.02 & 2.37 \\
\hline 181.49 & 3253.1 & 37.36 & 2.98 \\
\hline 181.72 & 3254.6 & 32.89 & 2.59 \\
\hline 182.06 & 3256.8 & 13.07 & 0.74 \\
\hline 182.32 & 3258.5 & 8.70 & 0.54 \\
\hline 182.60 & 3260.3 & 7.28 & 0.49 \\
\hline 182.88 & 3262.1 & 6.26 & 0.40 \\
\hline 183.17 & 3264.0 & 6.50 & 0.40 \\
\hline 183.45 & 3265.9 & 4.11 & 0.28 \\
\hline 183.74 & 3267.8 & 3.34 & 0.19 \\
\hline 184.02 & 3269.6 & 1.94 & 0.12 \\
\hline 184.31 & 3271.5 & 1.95 & 0.11 \\
\hline 184.59 & 3273.3 & 3.03 & 0.18 \\
\hline 184.88 & 3275.2 & 0.77 & 0.05 \\
\hline 185.16 & 3277.0 & 2.42 & 0.15 \\
\hline 185.45 & 3278.9 & 0.19 & 0.01 \\
\hline 185.73 & 3281.4 & 1.51 & 0.06 \\
\hline 185.98 & 3283.5 & 1.81 & 0.07 \\
\hline 186.28 & 3286.2 & 0.24 & 0.01 \\
\hline 186.57 & 3288.7 & 1.04 & 0.04 \\
\hline 186.80 & 3290.7 & 1.43 & 0.06 \\
\hline 187.15 & 3293.7 & 0.72 & 0.03 \\
\hline 187.42 & 3296.1 & 0.99 & 0.05 \\
\hline 187.72 & 3298.7 & 1.21 & 0.05 \\
\hline 188.01 & 3301.3 & 1.21 & 0.06 \\
\hline 188.24 & 3303.3 & 0.96 & 0.04 \\
\hline 188.39 & 3304.6 & 1.60 & 0.07 \\
\hline 188.63 & 3306.7 & 1.75 & 0.07 \\
\hline 188.87 & 3308.8 & 1.81 & 0.09 \\
\hline 189.17 & 3311.4 & 1.48 & 0.07 \\
\hline 189.46 & 3313.9 & 1.61 & 0.07 \\
\hline 189.69 & 3315.9 & 1.51 & 0.08 \\
\hline 190.03 & 3318.6 & 2.01 & 0.12 \\
\hline 190.31 & 3320.3 & 5.11 & 0.44 \\
\hline 190.61 & 3322.1 & 14.84 & 1.31 \\
\hline 190.90 & 3323.9 & 19.75 & 1.72 \\
\hline 191.13 & 3325.3 & 26.05 & 2.57 \\
\hline 191.19 & 3325.6 & 26.40 & 2.55 \\
\hline 191.48 & 3327.4 & 30.61 & 3.37 \\
\hline 191.76 & 3329.1 & 25.72 & 2.59 \\
\hline 192.05 & 3330.9 & 15.87 & 1.17 \\
\hline 192.34 & 3332.6 & 14.79 & 1.22 \\
\hline 192.57 & 3334.0 & 7.28 & 0.58 \\
\hline
\end{tabular}


Table 1 (continued).

\begin{tabular}{|c|c|c|c|c|c|c|c|c|c|}
\hline $\begin{array}{l}\text { Composite } \\
\text { depth } \\
\text { (m) }\end{array}$ & $\begin{array}{l}\text { Age } \\
\text { (ka) }\end{array}$ & $\begin{array}{l}\mathrm{CaCO}_{3} \\
\text { (wt } \% \text { ) }\end{array}$ & $\begin{array}{c}\text { MAR } \\
\mathrm{CaCO}_{3} \\
\left(\mathrm{~g} / \mathrm{cm}^{2} \mathrm{k} . \mathrm{y} .\right)\end{array}$ & $\begin{array}{l}\text { Composite } \\
\text { depth } \\
\text { (m) }\end{array}$ & $\begin{array}{l}\text { Age } \\
\text { (ka) }\end{array}$ & $\begin{array}{l}\mathrm{CaCO}_{3} \\
(\mathrm{wt} \%)\end{array}$ & $\begin{array}{c}\text { MAR } \\
\mathrm{CaCO}_{3} \\
\left(\mathrm{~g} / \mathrm{cm}^{2} \mathrm{k} . \mathrm{y} .\right)\end{array}$ & $\begin{array}{l}\text { Composite } \\
\text { depth } \\
\text { (m) }\end{array}$ & $\begin{array}{l}\text { Age } \\
\text { (ka) }\end{array}$ \\
\hline 192.92 & 3336.1 & 2.12 & 0.14 & 217.90 & 3541.9 & 0.00 & 0.00 & 240.23 & 3693.5 \\
\hline 193.20 & 3337.8 & 2.49 & 0.17 & 218.19 & 3544.5 & 0.08 & 0.00 & 240.55 & 3695.6 \\
\hline 193.52 & 3339.8 & 6.47 & 0.42 & 218.47 & 3546.9 & 1.52 & 0.07 & 240.87 & 3697.7 \\
\hline 193.80 & 3341.5 & 10.93 & 0.81 & 218.76 & 3549.3 & 3.35 & 0.16 & 241.19 & 3699.8 \\
\hline 194.09 & 3343.2 & 16.64 & 1.32 & 219.04 & 3551.6 & 8.20 & 0.48 & 241.73 & 3703.2 \\
\hline 194.37 & 3344.9 & 12.54 & 0.89 & 219.32 & 3553.8 & 18.75 & 0.91 & 241.83 & 3703.8 \\
\hline 194.65 & 3346.6 & 36.47 & 2.76 & 219.67 & 3556.5 & 10.93 & 0.46 & 242.15 & 3705.8 \\
\hline 194.94 & 3349.4 & 26.59 & 1.43 & 219.85 & 3557.9 & 0.06 & 0.00 & 242.48 & 3707.9 \\
\hline 195.28 & 3353.1 & 7.41 & 0.32 & 220.13 & 3560.2 & 0.09 & 0.00 & 242.90 & 3710.5 \\
\hline 195.58 & 3356.2 & 4.91 & 0.20 & 220.40 & 3562.3 & 0.09 & 0.00 & 243.13 & 3712.0 \\
\hline 195.82 & 3358.8 & 4.65 & 0.15 & 220.62 & 3564.0 & 0.00 & 0.00 & 243.23 & 3712.6 \\
\hline 195.88 & 3359.5 & 4.10 & 0.12 & 220.94 & 3566.5 & 0.10 & 0.00 & 243.47 & 3714.1 \\
\hline 196.18 & 3363.5 & 4.27 & 0.12 & 221.21 & 3568.7 & 0.04 & 0.00 & 243.75 & 3715.9 \\
\hline 196.48 & 3367.4 & 6.85 & 0.21 & 221.49 & 3570.9 & 0.03 & 0.00 & 244.04 & 3717.7 \\
\hline 196.78 & 3371.4 & 6.11 & 0.17 & 221.76 & 3573.0 & 0.15 & 0.01 & 244.32 & 3719.4 \\
\hline 197.08 & 3375.3 & 2.51 & 0.08 & 221.98 & 3574.7 & 0.00 & 0.00 & 244.55 & 3721.2 \\
\hline 197.32 & 3378.5 & 4.57 & 0.16 & 222.30 & 3577.3 & 0.00 & 0.00 & 244.89 & 3724.0 \\
\hline 197.68 & 3383.2 & 3.59 & 0.12 & 222.56 & 3579.2 & 0.00 & 0.00 & 245.18 & 3726.4 \\
\hline 197.98 & 3387.2 & 1.54 & 0.05 & 222.57 & 3579.3 & 0.00 & 0.00 & 245.46 & 3728.8 \\
\hline 198.28 & 3391.1 & 3.20 & 0.11 & 222.84 & 3580.9 & 0.00 & 0.00 & 245.75 & 3731.2 \\
\hline 198.58 & 3393.1 & 14.90 & 1.11 & 223.12 & 3582.6 & 0.44 & 0.04 & 245.98 & 3733.1 \\
\hline 198.82 & 3394.8 & 18.05 & 1.31 & 223.33 & 3583.8 & 1.62 & 0.13 & 246.12 & 3734.3 \\
\hline 198.88 & 3395.2 & 23.00 & 1.83 & 223.39 & 3584.2 & 2.50 & 0.18 & 246.32 & 3735.9 \\
\hline 199.18 & 3397.3 & 12.93 & 0.86 & 223.66 & 3585.8 & 4.08 & 0.35 & 246.60 & 3738.3 \\
\hline 199.48 & 3399.3 & 11.28 & 0.76 & 223.92 & 3587.4 & 14.07 & 1.01 & 246.89 & 3740.7 \\
\hline 199.78 & 3401.4 & 13.59 & 0.99 & 224.20 & 3589.1 & 20.18 & 1.57 & 247.17 & 3742.9 \\
\hline 200.08 & 3403.4 & 27.84 & 2.36 & 224.47 & 3590.7 & 13.94 & 1.16 & 247.40 & 3744.8 \\
\hline 200.08 & 3403.4 & 18.07 & 1.53 & 224.69 & 3592.0 & 7.39 & 0.64 & 248.03 & 3749.9 \\
\hline 200.32 & 3405.1 & 32.21 & 2.77 & 225.02 & 3594.0 & 2.74 & 0.23 & 248.30 & 3752.0 \\
\hline 200.68 & 3407.6 & 25.77 & 2.14 & 225.28 & 3595.5 & 1.66 & 0.12 & 248.60 & 3754.4 \\
\hline 200.98 & 3409.6 & 10.75 & 0.83 & 225.56 & 3597.2 & 1.10 & 0.08 & 248.83 & 3756.3 \\
\hline 201.28 & 3411.8 & 2.82 & 0.19 & 225.83 & 3598.8 & 1.46 & 0.11 & 248.88 & 3756.7 \\
\hline 201.82 & 3416.1 & 34.92 & 2.32 & 226.05 & 3600.1 & 1.63 & 0.11 & 249.17 & 3759.0 \\
\hline 201.88 & 3416.6 & 37.70 & 2.90 & 226.10 & 3600.4 & 1.80 & 0.13 & 249.45 & 3761.3 \\
\hline 202.18 & 3419.0 & 33.55 & 2.65 & 226.38 & 3602.1 & 1.46 & 0.09 & 249.74 & 3763.6 \\
\hline 202.48 & 3421.4 & 22.76 & 1.63 & 226.64 & 3603.6 & 0.53 & 0.04 & 250.02 & 3766.4 \\
\hline 202.78 & 3423.8 & 31.06 & 2.19 & 226.92 & 3605.1 & 0.46 & 0.03 & 250.25 & 3768.9 \\
\hline 203.08 & 3426.2 & 36.43 & 2.72 & 227.19 & 3606.5 & 0.23 & 0.02 & 250.59 & 3772.5 \\
\hline 203.32 & 3428.1 & 41.21 & 3.60 & 227.41 & 3607.6 & 0.62 & 0.05 & 250.88 & 3775.6 \\
\hline 203.68 & 3431.0 & 21.51 & 1.59 & 227.73 & 3609.2 & 1.29 & 0.08 & 251.15 & 3778.5 \\
\hline 203.95 & 3433.2 & 5.04 & 0.27 & 228.02 & 3610.7 & 1.18 & 0.10 & 251.44 & 3781.5 \\
\hline 204.24 & 3435.4 & 3.07 & 0.19 & 228.30 & 3612.1 & 0.49 & 0.04 & 251.72 & 3784.5 \\
\hline 204.58 & 3438.1 & 15.91 & 0.96 & 228.59 & 3613.6 & 0.49 & 0.04 & 252.01 & 3787.6 \\
\hline 204.86 & 3440.3 & 15.26 & 1.13 & 228.87 & 3615.0 & 1.57 & 0.11 & 252.29 & 3790.6 \\
\hline 205.14 & 3442.5 & 38.66 & 2.94 & 229.16 & 3616.4 & 1.80 & 0.14 & 252.58 & 3793.3 \\
\hline 205.37 & 3444.7 & 36.57 & 2.52 & 229.44 & 3617.8 & 2.93 & 0.20 & 252.87 & 3796.0 \\
\hline 205.43 & 3445.3 & 37.70 & 2.39 & 229.62 & 3618.8 & 3.74 & 0.32 & 253.15 & 3798.6 \\
\hline 205.71 & 3448.0 & 11.24 & 0.67 & 229.96 & 3620.5 & 2.06 & 0.16 & 253.43 & 3801.1 \\
\hline 205.99 & 3450.8 & 5.18 & 0.23 & 230.24 & 3621.9 & 1.85 & 0.13 & 253.72 & 3803.8 \\
\hline 206.28 & 3453.6 & 9.29 & 0.39 & 230.24 & 3621.9 & 1.53 & 0.11 & 254.01 & 3806.5 \\
\hline 206.56 & 3456.4 & 9.61 & 0.41 & 230.47 & 3623.0 & 2.30 & 0.21 & 254.18 & 3808.0 \\
\hline 206.79 & 3458.6 & 12.83 & 0.54 & 230.52 & 3623.3 & 4.20 & 0.30 & 254.47 & 3810.7 \\
\hline 207.13 & 3461.9 & 23.44 & 0.98 & 230.81 & 3624.9 & 4.07 & 0.32 & 254.70 & 3812.8 \\
\hline 207.41 & 3464.5 & 35.74 & 2.88 & 231.09 & 3626.4 & 7.76 & 0.54 & 254.76 & 3813.3 \\
\hline 207.70 & 3466.6 & 10.53 & 0.81 & 231.37 & 3628.0 & 7.84 & 0.57 & 255.04 & 3815.7 \\
\hline 207.98 & 3468.7 & 3.20 & 0.22 & 231.66 & 3629.6 & 5.86 & 0.41 & 255.33 & 3818.2 \\
\hline 208.21 & 3470.4 & 1.15 & 0.08 & 231.88 & 3630.9 & 6.00 & 0.46 & 255.62 & 3820.7 \\
\hline 208.26 & 3470.8 & 3.00 & 0.19 & 232.23 & 3632.9 & 2.49 & 0.18 & 255.91 & 3823.1 \\
\hline 208.55 & 3472.9 & 1.56 & 0.10 & 232.51 & 3634.4 & 2.26 & 0.18 & 256.14 & 3825.1 \\
\hline 208.83 & 3474.9 & 0.02 & 0.00 & 232.79 & 3636.0 & 0.62 & 0.05 & 256.49 & 3828.1 \\
\hline 209.12 & 3477.1 & 0.00 & 0.00 & 233.08 & 3637.6 & 1.77 & 0.12 & 256.78 & 3830.5 \\
\hline 209.40 & 3479.2 & 1.18 & 0.07 & 233.30 & 3638.9 & 1.18 & 0.09 & 257.06 & 3832.9 \\
\hline 209.63 & 3480.9 & 1.93 & 0.12 & 233.36 & 3639.2 & 1.20 & 0.08 & 257.35 & 3835.2 \\
\hline 209.97 & 3483.5 & 0.62 & 0.04 & 233.64 & 3640.8 & 2.07 & 0.16 & 257.59 & 3837.0 \\
\hline 210.25 & 3485.6 & 0.36 & 0.02 & 233.93 & 3642.4 & 3.70 & 0.21 & 257.64 & 3837.4 \\
\hline 210.54 & 3487.8 & 0.00 & 0.00 & 234.21 & 3644.6 & 6.12 & 0.30 & 257.93 & 3839.6 \\
\hline 211.05 & 3491.6 & 0.15 & 0.01 & 234.49 & 3647.2 & 7.20 & 0.32 & 258.22 & 3841.8 \\
\hline 211.10 & 3492.0 & 0.70 & 0.04 & 234.72 & 3649.2 & 7.20 & 0.36 & 258.51 & 3844.0 \\
\hline 211.67 & 3496.3 & 0.08 & 0.00 & 235.06 & 3652.3 & 4.08 & 0.22 & 258.80 & 3846.2 \\
\hline 211.96 & 3498.5 & 0.13 & 0.01 & 235.35 & 3654.9 & 1.64 & 0.08 & 259.08 & 3848.3 \\
\hline 212.24 & 3500.6 & 0.24 & 0.02 & 235.63 & 3657.5 & 7.40 & 0.34 & 259.37 & 3850.5 \\
\hline 212.47 & 3502.3 & 0.29 & 0.02 & 235.91 & 3660.0 & 7.73 & 0.37 & 259.66 & 3852.7 \\
\hline 213,09 & 3506.8 & 12.68 & 0.81 & 236.14 & 3662.1 & 6.67 & 0.32 & 259.95 & 3854.9 \\
\hline 213.38 & 3508.7 & 11.47 & 0.78 & 236.29 & 3663.4 & 8.20 & 0.36 & 260.24 & 3857.0 \\
\hline 213.66 & 3510.4 & 7.03 & 0.46 & 236.48 & 3665.2 & 10.93 & 0.60 & 260.47 & 3858.7 \\
\hline 213.85 & 3511.7 & 1.89 & 0.12 & 236.76 & 3667.7 & 4.70 & 0.27 & 260.52 & 3859.1 \\
\hline 214.14 & 3513.5 & 0.70 & 0.04 & 237.05 & 3670.3 & 8.69 & 0.49 & 260.82 & 3861.3 \\
\hline 214.43 & 3515.3 & 0.68 & 0.04 & 237.33 & 3672.9 & 4.33 & 0.21 & 261.11 & 3863.5 \\
\hline 214.71 & 3517.1 & 0.22 & 0.01 & 237.56 & 3674.9 & 1.92 & 0.10 & 261.39 & 3865.6 \\
\hline 215.04 & 3519.2 & 0.16 & 0.01 & 237.90 & 3678.0 & 0.21 & 0.01 & 261.68 & 3867.7 \\
\hline 215.33 & 3521.1 & 0.14 & 0.01 & 238.18 & 3680.1 & 1.12 & 0.07 & 261.90 & 3869.4 \\
\hline 215.62 & 3522.9 & 0.65 & 0.04 & 238.46 & 3682.0 & 3.48 & 0.18 & 262.26 & 3872.1 \\
\hline 215.90 & 3524.7 & 0.28 & 0.01 & 238.65 & 3683.2 & 2.70 & 0.20 & 262.54 & 3874.1 \\
\hline 216.47 & 3529.2 & 0.15 & 0.01 & 238.73 & 3683.7 & 6.01 & 0.41 & 262.81 & 3876.2 \\
\hline 216.76 & 3531.8 & 0.03 & 0.00 & 238.94 & 3685.1 & 8.58 & 0.52 & 263.08 & 3878.2 \\
\hline 217.04 & 3534.3 & 0.04 & 0.00 & 239.26 & 3687.2 & 1.09 & 0.07 & 263.36 & 3880.2 \\
\hline 217.33 & 3536.8 & 0.00 & 0.00 & 239.59 & 3689.4 & 1.58 & 0.11 & 263.59 & 3882.0 \\
\hline 217.62 & 3539.4 & 0.04 & 0.00 & 239.94 & 3691.6 & 0.53 & 0.04 & 263.64 & 3882.3 \\
\hline
\end{tabular}


Table 1 (continued).

\begin{tabular}{|c|c|c|c|}
\hline $\begin{array}{l}\text { Composite } \\
\text { depth } \\
\text { (m) }\end{array}$ & $\begin{array}{l}\text { Age } \\
\text { (ka) }\end{array}$ & $\begin{array}{c}\mathrm{CaCO}_{3} \\
\text { (wt\%) }\end{array}$ & $\begin{array}{c}\text { MAR } \\
\mathrm{CaCO}_{3} \\
\left(\mathrm{~g} / \mathrm{cm}^{2} \text { k.y. }\right)\end{array}$ \\
\hline 263.93 & 3885.0 & 0.26 & 0.01 \\
\hline 264.21 & 3887.8 & 1.36 & 0.04 \\
\hline 264.50 & 3890.6 & 1.60 & 0.06 \\
\hline 265.01 & 3895.5 & 2.81 & 0.15 \\
\hline 265.36 & 3897.8 & 1.38 & 0.08 \\
\hline 265.64 & 3899.7 & 0.77 & 0.04 \\
\hline 265.93 & 3901.6 & 0.78 & 0.05 \\
\hline 266.21 & 3903.5 & 0.19 & 0.01 \\
\hline 266.44 & 3905.0 & 8.12 & 0.46 \\
\hline 266.50 & 3905.4 & 0.00 & 0.00 \\
\hline 266.78 & 3907.3 & 1.06 & 0.07 \\
\hline 267.07 & 3909.3 & 5.92 & 0.38 \\
\hline 267.35 & 3911.1 & 12.14 & 0.86 \\
\hline 267.64 & 3913.1 & 6.29 & 0.50 \\
\hline 267.86 & 3914.5 & 3.93 & 0.27 \\
\hline 268.21 & 3916.9 & 1.18 & 0.08 \\
\hline 268.49 & 3918.8 & 2.54 & 0.18 \\
\hline 268.78 & 3920.7 & 5.34 & 0.36 \\
\hline 268.97 & 3922.0 & 0.27 & 0.02 \\
\hline 269.29 & 3924.1 & 1.61 & 0.10 \\
\hline 269.35 & 3924.5 & 1.60 & 0.11 \\
\hline 269.63 & 3926.4 & 2.43 & 0.16 \\
\hline 269.92 & 3928.3 & 2.68 & 0.17 \\
\hline 270.20 & 3930.2 & 0.55 & 0.03 \\
\hline 270.49 & 3932.2 & 2.53 & 0.16 \\
\hline 270.71 & 3933.7 & 0.21 & 0.02 \\
\hline 271.06 & 3936.1 & 1.51 & 0.10 \\
\hline 271.34 & 3938.1 & 1.35 & 0.08 \\
\hline 271.74 & 3940.8 & 1.57 & 0.09 \\
\hline 271.91 & 3942.0 & 1.27 & 0.07 \\
\hline 272.20 & 3944.0 & 1.36 & 0.08 \\
\hline 272.50 & 3946.0 & 1.26 & 0.07 \\
\hline 272.80 & 3948.1 & 0.96 & 0.06 \\
\hline 273.10 & 3950.1 & 0.59 & 0.03 \\
\hline 273.39 & 3952.1 & 0.96 & 0.05 \\
\hline 273.69 & 3954.2 & 1.73 & 0.10 \\
\hline 273.99 & 3956.3 & 0.64 & 0.03 \\
\hline 274.28 & 3958.2 & 1.40 & 0.09 \\
\hline 274.65 & 3960.8 & 0.71 & 0.04 \\
\hline 274.70 & 3961.1 & 2.20 & 0.12 \\
\hline 274.99 & 3963.1 & 0.75 & 0.04 \\
\hline 275.27 & 3965.0 & 0.47 & 0.02 \\
\hline 275.57 & 3967.5 & 0.33 & 0.01 \\
\hline 275.86 & 3970.1 & 0.15 & 0.01 \\
\hline 276.09 & 3972.2 & 0.23 & 0.01 \\
\hline 276.44 & 3975.5 & 0.34 & 0.02 \\
\hline 276.72 & 3978.0 & 1.03 & 0.05 \\
\hline 277.01 & 3980.7 & 1.42 & 0.06 \\
\hline 277.30 & 3983.3 & 2.92 & 0.13 \\
\hline 277.53 & 3985.5 & 3.05 & 0.15 \\
\hline 277.59 & 3986.0 & 3.80 & 0.17 \\
\hline 277.88 & 3988.7 & 3.38 & 0.15 \\
\hline 278.16 & 3991.2 & 2.54 & 0.11 \\
\hline 278.46 & 3994.0 & 3.34 & 0.14 \\
\hline 278.75 & 3996.6 & 0.28 & 0.01 \\
\hline 278.98 & 3998.8 & 0.46 & 0.02 \\
\hline 279.32 & 4001.9 & 0.84 & 0.03 \\
\hline 279.60 & 4004.4 & 0.23 & 0.01 \\
\hline 279.90 & 4007.2 & 0.49 & 0.02 \\
\hline 280.19 & 4009.9 & 0.83 & 0.03 \\
\hline 280.42 & 4012.0 & 1.30 & 0.05 \\
\hline 280.48 & 4012.5 & 1.20 & 0.05 \\
\hline 280.77 & 4015.2 & 0.79 & 0.04 \\
\hline 281.05 & 4017.7 & 1.43 & 0.06 \\
\hline 281.34 & 4020.4 & 0.20 & 0.01 \\
\hline 281.63 & 4023.1 & 0.14 & 0.01 \\
\hline 281.86 & 4025.2 & 0.14 & 0.01 \\
\hline 282.21 & 4028.4 & 0.97 & 0.05 \\
\hline 282.49 & 4030.9 & 0.92 & 0.05 \\
\hline 283.05 & 4036.1 & 0.47 & 0.02 \\
\hline 283.36 & 4038.9 & 0.54 & 0.02 \\
\hline 283.63 & 4041.4 & 0.87 & 0.04 \\
\hline 283.92 & 4044.1 & 0.81 & 0.04 \\
\hline 284.22 & 4046.8 & 0.49 & 0.02 \\
\hline 284.51 & 4049.5 & 1.31 & 0.06 \\
\hline 284.82 & 4052.3 & 0.07 & 0.00 \\
\hline 285.09 & 4054.8 & 0.11 & 0.01 \\
\hline 285.38 & 4057.5 & 1.41 & 0.07 \\
\hline 285.67 & 4060.1 & 1.99 & 0.10 \\
\hline 285.96 & 4062.8 & 1.69 & 0.08 \\
\hline 286.27 & 4065.6 & 1.11 & 0.05 \\
\hline 286.55 & 4068.2 & 0.45 & 0.02 \\
\hline 286.84 & 4070.8 & 1.42 & 0.06 \\
\hline 287.13 & 4073.5 & 2.10 & 0.09 \\
\hline 287.42 & 4076.2 & 0.92 & 0.04 \\
\hline 287.85 & 4080.1 & 0.80 & 0.03 \\
\hline 288.15 & 4082.9 & 0.45 & 0.02 \\
\hline 288.45 & 4085.6 & 0.17 & 0.01 \\
\hline
\end{tabular}

\begin{tabular}{|c|c|c|c|}
\hline $\begin{array}{l}\text { Composite } \\
\text { depth } \\
\text { (m) }\end{array}$ & $\begin{array}{l}\text { Age } \\
\text { (ka) }\end{array}$ & $\begin{array}{l}\mathrm{CaCO}_{3} \\
\text { (wt\%) }\end{array}$ & $\begin{array}{c}\text { MAR } \\
\mathrm{CaCO}_{3} \\
\left(\mathrm{~g} / \mathrm{cm}^{2} \mathrm{k} . \mathrm{y} .\right)\end{array}$ \\
\hline 288.69 & 4087.8 & 0.25 & 0.01 \\
\hline 288.75 & 4088.4 & 0.00 & 0.00 \\
\hline 289.05 & 4091.1 & 0.24 & 0.01 \\
\hline 289.35 & 4093.9 & 0.82 & 0.04 \\
\hline 289.65 & 4096.6 & 0.88 & 0.04 \\
\hline 289.95 & 4099.4 & 1.09 & 0.04 \\
\hline 290.19 & 4101.6 & 0.51 & 0.02 \\
\hline 290.55 & 4104.9 & 0.32 & 0.01 \\
\hline 290.85 & 4107.6 & 1.07 & 0.05 \\
\hline 291.15 & 4110.4 & 1.49 & 0.06 \\
\hline 291.45 & 4113.1 & 1.34 & 0.06 \\
\hline 291.69 & 4115.3 & 0.32 & 0.01 \\
\hline 291.75 & 4115.9 & 1.00 & 0.05 \\
\hline 292.05 & 4118.6 & 1.68 & 0.08 \\
\hline 292.35 & 4121.4 & 0.43 & 0.02 \\
\hline 292.65 & 4124.1 & 1.47 & 0.07 \\
\hline 292.95 & 4126.9 & 0.16 & 0.01 \\
\hline 293.19 & 4129.1 & 0.19 & 0.01 \\
\hline 293.55 & 4132.4 & 0.24 & 0.01 \\
\hline 293.85 & 4135.2 & 0.20 & 0.01 \\
\hline 294.15 & 4137,9 & 0.57 & 0.03 \\
\hline 294.45 & 4140.7 & 0.83 & 0.04 \\
\hline 294.69 & 4142.9 & 2.04 & 0.10 \\
\hline 294.75 & 4143.4 & 2.10 & 0.10 \\
\hline 295.05 & 4146.2 & 0.88 & 0.05 \\
\hline 295.35 & 4148.9 & 0.17 & 0.01 \\
\hline 295.65 & 4151.7 & 0.46 & 0.02 \\
\hline 295.95 & 4154.4 & 0.85 & 0.05 \\
\hline 296.05 & 4155.3 & 0.10 & 0.00 \\
\hline 296.19 & 4156.6 & 0.25 & 0.01 \\
\hline 296.55 & 4159.9 & 1.75 & 0.08 \\
\hline 296.85 & 4162.7 & 1.40 & 0.07 \\
\hline 297.15 & 4165.4 & 0.50 & 0.02 \\
\hline 297.35 & 4167.3 & 0.03 & 0.00 \\
\hline 297.65 & 4170.0 & 0.39 & 0.02 \\
\hline 297.95 & 4172.8 & 0.46 & 0.02 \\
\hline 298.19 & 4175.0 & 2.71 & 0.15 \\
\hline 298.26 & 4175.6 & 3.10 & 0.16 \\
\hline 298.55 & 4178.3 & 1.38 & 0.06 \\
\hline 298.85 & 4181.3 & 2.96 & 0.12 \\
\hline 299.15 & 4184.7 & 4.44 & 0.18 \\
\hline 299.45 & 4188.2 & 1.22 & 0.05 \\
\hline 299.69 & 4191.0 & 0.86 & 0.03 \\
\hline 300.05 & 4195.2 & 2.11 & 0.08 \\
\hline 300.35 & 4198.6 & 0.44 & 0.02 \\
\hline 300.65 & 4202.1 & 7.52 & 0.26 \\
\hline 300.95 & 4205.6 & 2.06 & 0.08 \\
\hline 301.19 & 4208.4 & 0.09 & 0.00 \\
\hline 301.26 & 4209.2 & 0.00 & 0.00 \\
\hline 301.55 & 4212.5 & 0.07 & 0.00 \\
\hline 301.85 & 4216.0 & 0.08 & 0.00 \\
\hline 302.15 & 4219.5 & 0.87 & 0.04 \\
\hline 302.45 & 4222.9 & 3.26 & 0.14 \\
\hline 302.69 & 4225.7 & 8.09 & 0.35 \\
\hline 303.05 & 4229.9 & 1.79 & 0.08 \\
\hline 303.35 & 4233.3 & 2.35 & 0.09 \\
\hline 303.65 & 4236.8 & 0.37 & 0.01 \\
\hline 303.95 & 4240.3 & 3.64 & 0.15 \\
\hline 304.19 & 4243.1 & 2.10 & 0.09 \\
\hline 304.26 & 4243.9 & 7.00 & 0.32 \\
\hline 304.55 & 4247.2 & 2.35 & 0.10 \\
\hline 304.85 & 4250.7 & 1.17 & 0.05 \\
\hline 305.15 & 4254.2 & 0.79 & 0.03 \\
\hline 305.45 & 4257.6 & 5.84 & 0.27 \\
\hline 305.69 & 4260.4 & 3.56 & 0.13 \\
\hline 306.05 & 4264.6 & 4.76 & 0.18 \\
\hline 306.35 & 4268.1 & 7.05 & 0.27 \\
\hline 306.65 & 4271.5 & 2.40 & 0.09 \\
\hline 307.69 & 4283.6 & 2.80 & 0.08 \\
\hline 308.35 & 4291.2 & 5.56 & 0.19 \\
\hline 309.85 & 4308.6 & 12.90 & 0.48 \\
\hline 310.15 & 4312.0 & 11.87 & 0.37 \\
\hline 310.45 & 4315.5 & 8.47 & 0.31 \\
\hline 310.69 & 4318.3 & 5.07 & 0.19 \\
\hline 310.78 & 4319.3 & 4.50 & 0.20 \\
\hline 311.05 & 4321.5 & 1.68 & 0.09 \\
\hline 311.35 & 4323.6 & 1.78 & 0.10 \\
\hline 311.65 & 4325.7 & 0.55 & 0.04 \\
\hline 311.95 & 4327.8 & 0.45 & 0.02 \\
\hline 312.19 & 4329.5 & 1.11 & 0.07 \\
\hline 312.55 & 4332.0 & 2.32 & 0.13 \\
\hline 312.85 & 4334.1 & 5.43 & 0.31 \\
\hline 313.15 & 4336.2 & 1.45 & 0.10 \\
\hline 313.45 & 4338.3 & 0.71 & 0.05 \\
\hline 313.69 & 4340.0 & 0.55 & 0.03 \\
\hline 313.75 & 4340.4 & 0.00 & 0.00 \\
\hline 314.05 & 4342.5 & 0.33 & 0.02 \\
\hline 314.35 & 4344.6 & 0.10 & 0.01 \\
\hline
\end{tabular}

\begin{tabular}{|c|c|c|c|}
\hline $\begin{array}{l}\text { Composite } \\
\text { depth } \\
\text { (m) }\end{array}$ & $\begin{array}{l}\text { Age } \\
\text { (ka) }\end{array}$ & $\begin{array}{l}\mathrm{CaCO}_{3} \\
(\mathrm{wt} \%)\end{array}$ & $\begin{array}{c}\text { MAR } \\
\mathrm{CaCO}_{3} \\
\left(\mathrm{~g} / \mathrm{cm}^{2} \mathrm{k} . \mathrm{y} .\right)\end{array}$ \\
\hline 314.65 & 4346.7 & 0.13 & 0.01 \\
\hline 315.05 & 4349.5 & 5.99 & 0.41 \\
\hline 315.35 & 4351.6 & 5.10 & 0.34 \\
\hline 315.65 & 4353.7 & 3.55 & 0.21 \\
\hline 316.35 & 4358.6 & 0.26 & 0.02 \\
\hline 316.65 & 4360.7 & 0.32 & 0.02 \\
\hline 316.95 & 4362.8 & 0.79 & 0.04 \\
\hline 317.19 & 4364.5 & 0.71 & 0.04 \\
\hline 317.25 & 4364.9 & 1.60 & 0.09 \\
\hline 317.55 & 4367.0 & 0.64 & 0.04 \\
\hline 317.85 & 4369.1 & 0.42 & 0.02 \\
\hline 318.15 & 4371.2 & 4.08 & 0.26 \\
\hline 318.45 & 4373.3 & 1.59 & 0.11 \\
\hline 318.69 & 4375.0 & 0.57 & 0.03 \\
\hline 319.05 & 4377.6 & 2.71 & 0.17 \\
\hline 319.35 & 4379.7 & 1.23 & 0.06 \\
\hline 319.65 & 4381.8 & 1.24 & 0.07 \\
\hline 319.95 & 4383.9 & 0.25 & 0.02 \\
\hline 320.19 & 4385.5 & 1.34 & 0.07 \\
\hline 320.25 & 4386.0 & 3.20 & 0.18 \\
\hline 320.55 & 4388.1 & 3.74 & 0.22 \\
\hline 320.85 & 4390.2 & 5.61 & 0.37 \\
\hline 321.15 & 4392.3 & 2.28 & 0.16 \\
\hline 321.45 & 4394.4 & 3.93 & 0.28 \\
\hline 321.69 & 4396.1 & 1.79 & 0.10 \\
\hline 322.05 & 4398.6 & 0.82 & 0.05 \\
\hline 322.35 & 4400.7 & 2.49 & 0.16 \\
\hline 322.65 & 4402.8 & 0.83 & 0.05 \\
\hline 322.95 & 4404.9 & 0.25 & 0.01 \\
\hline 323.19 & 4406.6 & 0.30 & 0.02 \\
\hline 323.25 & 4407.0 & 0.00 & 0.00 \\
\hline 323.55 & 4409.1 & 0.13 & 0.01 \\
\hline 323.85 & 4411.2 & 0.09 & 0.01 \\
\hline 324.15 & 4413.3 & 0.13 & 0.01 \\
\hline 324.45 & 4415.4 & 0.20 & 0.01 \\
\hline 324.69 & 4417.1 & 0.90 & 0.05 \\
\hline 325.05 & 4419.6 & 0.08 & 0.00 \\
\hline 325.35 & 4421.7 & 4.65 & 0.25 \\
\hline 325.57 & 4423.3 & 0.94 & 0.06 \\
\hline 325.85 & 4425.2 & 1.87 & 0.10 \\
\hline 326.15 & 4427.3 & 0.44 & 0.03 \\
\hline 326.45 & 4429.4 & 0.08 & 0.00 \\
\hline 326.69 & 4431.1 & 0.25 & 0.01 \\
\hline 326.75 & 4431.5 & 1.70 & 0.09 \\
\hline 327.05 & 4433.6 & 0.57 & 0.03 \\
\hline 327.35 & 4435.7 & 0.09 & 0.00 \\
\hline 327.65 & 4437.8 & 0.21 & 0.01 \\
\hline 327.95 & 4439.9 & 0.11 & 0.01 \\
\hline 328.19 & 4441.6 & 0.11 & 0.01 \\
\hline 328.55 & 4444.1 & 0.09 & 0.00 \\
\hline 328.85 & 4446.3 & 0.07 & 0.00 \\
\hline 329.15 & 4448.4 & 0.12 & 0.01 \\
\hline 329.45 & 4450.5 & 0.19 & 0.01 \\
\hline 329.69 & 4452.1 & 0.14 & 0.01 \\
\hline 329.74 & 4452.5 & 0.00 & 0.00 \\
\hline 330.05 & 4454.7 & 0.09 & 0.01 \\
\hline 330.35 & 4456.8 & 0.39 & 0.02 \\
\hline 330.65 & 4458.9 & 1.88 & 0.12 \\
\hline 330.95 & 4461.0 & 3.76 & 0.22 \\
\hline 331.19 & 4462.7 & 1.99 & 0.10 \\
\hline 331.55 & 4465.2 & 3.50 & 0.22 \\
\hline 331.85 & 4467.3 & 0.18 & 0.01 \\
\hline 332.15 & 4469.4 & 0.18 & 0.01 \\
\hline 332.45 & 4471.5 & 0.26 & 0.02 \\
\hline 332.69 & 4473.2 & 0.13 & 0.01 \\
\hline 332.75 & 4473.6 & 0.00 & 0.00 \\
\hline 333.05 & 4475.7 & 0.14 & 0.01 \\
\hline 333.35 & 4477.8 & 0.04 & 0.00 \\
\hline 333.65 & 4480.2 & 0.07 & 0.00 \\
\hline 333.95 & 4482.7 & 0.11 & 0.01 \\
\hline 334.19 & 4484.7 & 0.08 & 0.00 \\
\hline 334.55 & 4487.6 & 0.06 & 0.00 \\
\hline 334.85 & 4490.1 & 0.08 & 0.00 \\
\hline 335.15 & 4492.6 & 0.15 & 0.01 \\
\hline 335.35 & 4494.2 & 0.07 & 0.00 \\
\hline 335.67 & 4496.9 & 0.48 & 0.02 \\
\hline 335.95 & 4499.2 & 0.20 & 0.01 \\
\hline 336.19 & 4501.1 & 0.77 & 0.04 \\
\hline 336.24 & 4501.6 & 0.00 & 0.00 \\
\hline 336.55 & 4504.1 & 0.41 & 0.02 \\
\hline 336.85 & 4506.6 & 0.52 & 0.03 \\
\hline 337.15 & 4509.1 & 0.25 & 0.01 \\
\hline 337.45 & 4511.5 & 0.23 & 0.01 \\
\hline 337.69 & 4513.5 & 0.70 & 0.04 \\
\hline 338.05 & 4516.5 & 0.06 & 0.00 \\
\hline 338.35 & 4518.9 & 0.10 & 0.00 \\
\hline 338.65 & 4521.4 & 0.06 & 0.00 \\
\hline 338.95 & 4523.9 & 0.07 & 0.00 \\
\hline
\end{tabular}


Table 1 (continued).

\begin{tabular}{|c|c|c|c|c|c|c|c|c|c|}
\hline $\begin{array}{l}\text { Composite } \\
\text { depth } \\
\text { (m) }\end{array}$ & $\begin{array}{l}\text { Age } \\
\text { (ka) }\end{array}$ & $\begin{array}{l}\mathrm{CaCO}_{3} \\
\text { (wt\%) }\end{array}$ & $\begin{array}{c}\text { MAR } \\
\mathrm{CaCO}_{3} \\
\left(\mathrm{~g} / \mathrm{cm}^{2} \mathrm{k} . \mathrm{y} .\right)\end{array}$ & $\begin{array}{l}\text { Composite } \\
\text { depth } \\
\text { (m) }\end{array}$ & $\begin{array}{l}\text { Age } \\
\text { (ka) }\end{array}$ & $\begin{array}{l}\mathrm{CaCO}_{3} \\
(\mathrm{wt} \%)\end{array}$ & $\begin{array}{c}\text { MAR } \\
\mathrm{CaCO}_{3} \\
\left(\mathrm{~g} / \mathrm{cm}^{2} \mathrm{k} . \mathrm{y} .\right)\end{array}$ & $\begin{array}{l}\text { Composite } \\
\text { depth } \\
\text { (m) }\end{array}$ & $\begin{array}{l}\text { Age } \\
\text { (ka) }\end{array}$ \\
\hline 339.19 & 4525.9 & 0.08 & 0.00 & 363.85 & 4826.8 & 0.30 & 0.01 & 388.55 & 5224.2 \\
\hline 339.24 & 4526.3 & 0.00 & 0.00 & 364.15 & 4831.0 & 0.74 & 0.02 & 388.85 & 5229.2 \\
\hline 339.55 & 4528.8 & 0.13 & 0.01 & 364.45 & 4835.1 & 0.19 & 0.01 & 389.15 & 5234.2 \\
\hline 339.85 & 4531.3 & 0.15 & 0.01 & 364.69 & 4838.5 & 0.25 & 0.01 & 389.45 & 5239.2 \\
\hline 340.15 & 4533.8 & 0.06 & 0.00 & 364.75 & 4839.3 & 1.20 & 0.03 & 389.69 & 5243.2 \\
\hline 340.45 & 4536.2 & 0.29 & 0.01 & 365.05 & 4843.5 & 0.37 & 0.01 & 389.76 & 5244.3 \\
\hline 340.69 & 4538.2 & 0.25 & 0.01 & 365.35 & 4847.6 & 2.98 & 0.10 & 390.05 & 5249.2 \\
\hline 341.05 & 4541.2 & 0.96 & 0.04 & 365.65 & 4851.8 & 6.08 & 0.22 & 390.35 & 5254.2 \\
\hline 341.35 & 4543.6 & 0.25 & 0.01 & 365.95 & 4856.0 & 10.62 & 0.37 & 390.65 & 5259.2 \\
\hline 341.65 & 4546.1 & 0.73 & 0.04 & 366.19 & 4859.3 & 3.93 & 0.13 & 390.95 & 5264.2 \\
\hline 341.95 & 4548.6 & 1.94 & 0.11 & 366.55 & 4864.3 & 5.97 & 0.22 & 391.19 & 5268.2 \\
\hline 342.19 & 4550.6 & 0.97 & 0.05 & 366.85 & 4868.5 & 2.64 & 0.10 & 391.55 & 5274.2 \\
\hline 342.24 & 4551.0 & 2.00 & 0.11 & 367.15 & 4872.6 & 4.43 & 0.16 & 391.85 & 5279.2 \\
\hline 342.55 & 4553.5 & 0.29 & 0.02 & 367.45 & 4876.8 & $\begin{array}{l}4.43 \\
4.48\end{array}$ & 0.17 & 392.65 & 5292.5 \\
\hline 342.85 & 4556.0 & 0.84 & 0.05 & 367.69 & 4880.2 & 0.25 & 0.01 & 392.95 & 5297.5 \\
\hline 343.15 & 4558.5 & 0.09 & 0.01 & 367.75 & 4881.1 & 1.70 & 0.05 & 393.19 & 5301.5 \\
\hline 343.45 & 4560.9 & 0.17 & 0.01 & 368.05 & 4886.0 & 2.38 & 0.08 & 393.26 & 5302.7 \\
\hline 343.69 & 4562.9 & 0.09 & 0.00 & 368.35 & 4890.8 & 0.69 & 0.02 & 393.55 & 5307.5 \\
\hline 344.05 & 4565.9 & 0.09 & 0.00 & 368.65 & 4895.6 & 0.07 & 0.00 & 393.85 & 5312.5 \\
\hline 344.85 & 4572.5 & 0.14 & 0.01 & 368.95 & $\begin{array}{l}4900.4 \\
\end{array}$ & 2.38 & 0.07 & 394.15 & 5317.5 \\
\hline 345.15 & 4574.9 & 1.54 & 0.08 & 369.19 & 4904.3 & 2.08 & 0.06 & 394.45 & 5322.5 \\
\hline 345.45 & 4577.4 & 0.21 & 0.01 & 369.55 & 4910.1 & 1.42 & 0.05 & 394.69 & 5326.5 \\
\hline 345.69 & 4579.4 & 0.31 & 0.01 & 369.85 & 4914.9 & 0.26 & 0.01 & 395.05 & 5332.5 \\
\hline 345.75 & 4579.9 & 1.00 & 0.05 & 370.15 & 4919.8 & 15.84 & 0.56 & 395.35 & 5337.5 \\
\hline 346.35 & 4584.8 & 0.45 & 0.02 & 370.45 & 4924.6 & $\begin{array}{r}8.04 \\
8.27\end{array}$ & 0.27 & 395.65 & 5342.5 \\
\hline 346.65 & 4587.3 & 0.06 & 0.00 & 370.69 & 4928.5 & $\begin{array}{l}0.27 \\
4.77\end{array}$ & 0.13 & 395.95 & 5347.6 \\
\hline 346.90 & 4589.3 & 0.09 & 0.00 & 370.75 & 4929.4 & 5.20 & 0.15 & 396.19 & 5351.6 \\
\hline 346.95 & 4589.8 & 0.09 & 0.00 & 371.05 & 4934.3 & 1.70 & 0.05 & 396.26 & 5352.7 \\
\hline 347.19 & 4591.7 & 0.11 & 0.01 & 371.35 & 4939.1 & 3.84 & 0.13 & 396.55 & 5357.6 \\
\hline 347.55 & 4594.7 & 0.04 & 0.00 & 371.65 & 4943.9 & $\begin{array}{l}3.07 \\
5.89\end{array}$ & 0.21 & 396.85 & 5362.6 \\
\hline 347.85 & 4597.2 & 0.18 & 0.01 & 371.95 & 4948.7 & 22.90 & 0.85 & 397.15 & 5367.6 \\
\hline 348.15 & 4599.6 & 0.62 & 0.04 & 372.19 & 4952.6 & 30.04 & 1.12 & 397.45 & 5372.6 \\
\hline 348.40 & 4601.7 & 0.09 & 0.00 & 372.55 & 4958.4 & 39.71 & 1.72 & 397.69 & 5376.6 \\
\hline 348.45 & 4602.1 & 0.91 & 0.05 & 372.85 & $\begin{array}{r}4963.4 \\
4963.2\end{array}$ & 23.85 & 0.94 & 398.05 & 5382.6 \\
\hline 348.69 & 4604.2 & 0.77 & 0.04 & 373.15 & 4968.1 & 38.60 & 0.87 & 398.35 & 5387.6 \\
\hline 348.75 & 4605.0 & 0.00 & 0.00 & 373.35 & 4971.3 & $\begin{array}{r}2.10 \\
2.10\end{array}$ & 0.06 & 398.65 & 5392.6 \\
\hline 349.05 & 4609.5 & 0.08 & 0.00 & 373.64 & 4975.9 & $\begin{array}{l}11.10 \\
\end{array}$ & 0.36 & 398.95 & 5397.6 \\
\hline 349.35 & 4614.0 & 0.42 & 0.01 & 373.95 & 4981.0 & 0.89 & 0.02 & 399.19 & 5401.6 \\
\hline 349.65 & 4618.4 & 0.06 & 0.00 & 374.18 & 4984.8 & 5.51 & 0.15 & 399.26 & 5402.7 \\
\hline 349.95 & 4622.9 & 1.37 & 0.05 & 374.25 & 4986.0 & 9.70 & 0.29 & 399.55 & 5407.6 \\
\hline 350.19 & 4626.5 & 0.11 & 0.00 & 374.55 & 4991.0 & 4.11 & 0.13 & 399.85 & 5412.6 \\
\hline 350.55 & 4631.8 & 0.14 & 0.00 & 375.14 & 5000.8 & $\begin{array}{r}4.11 \\
10.25\end{array}$ & 0.36 & 400.59 & 5424.9 \\
\hline 350.85 & 4636.3 & 0.10 & 0.00 & 375.44 & 5005.8 & 24.60 & 0.83 & 401.84 & 5445.8 \\
\hline 351.15 & 4640.8 & 0.11 & 0.00 & 375.69 & 5010.0 & 13.79 & 0.41 & 402.15 & 5450.9 \\
\hline 351.45 & 4645.2 & 0.07 & 0.00 & 376.05 & 5016.0 & 28.76 & $\begin{array}{l}.41 \\
1.02\end{array}$ & 402.45 & 5455.9 \\
\hline 351.75 & 4649.7 & 0.00 & 0.00 & 376.35 & 5021.0 & 36.77 & 1.47 & 402.68 & 5459.8 \\
\hline 352.05 & 4654.2 & 0.11 & 0.00 & 376.64 & 5025.8 & 24.57 & 0.96 & 402.75 & 5460.9 \\
\hline 352.35 & 4658.6 & 0.12 & 0.00 & 376.94 & 5030.8 & 13.89 & 0.42 & 403.05 & 5465.9 \\
\hline 352.65 & 4663.1 & 0.11 & 0.00 & 377.19 & 5035.0 & 6.89 & 0.23 & 403.09 & 5466.6 \\
\hline 352.95 & 4667.6 & 0.32 & 0.01 & 377.25 & 5036.0 & 2.30 & 0.07 & 403.34 & 5470.8 \\
\hline 353.19 & 4671.2 & 0.19 & 0.01 & 377.55 & 5041.0 & $\begin{array}{l}2.50 \\
1.68\end{array}$ & 0.04 & 403.65 & 5475.9 \\
\hline 353.55 & 4676.5 & 0.09 & 0.00 & 377.85 & 5046.0 & $\begin{array}{l}1.00 \\
22.58\end{array}$ & 0.83 & 403.95 & 5480.9 \\
\hline 353.85 & 4681.0 & 0.13 & 0.00 & 378.14 & 5050.8 & 29.40 & 1.12 & 404.18 & 5484.8 \\
\hline 354.15 & 4685.4 & 0.15 & 0.00 & 378.44 & 5055.8 & 35.00 & 1.29 & 404.55 & 5490.9 \\
\hline 354.35 & 4688.4 & 0.22 & 0.01 & 378.69 & 5060.0 & 50.34 & 2.24 & 404.84 & 5495.8 \\
\hline 354.65 & 4692.9 & 0.05 & 0.00 & 379.05 & 5065.9 & 51.94 & 2.35 & 405.15 & 5500.9 \\
\hline 354.95 & 4697.4 & 0.96 & 0.03 & 379.35 & 5070.9 & $\begin{array}{l}31.94 \\
45.51\end{array}$ & 1.68 & 405.45 & 5505.9 \\
\hline 355.19 & 4700.9 & 0.19 & 0.01 & 379.64 & 5075.8 & $\begin{array}{r}7.01 \\
8.93\end{array}$ & $\begin{array}{l}1.00 \\
0.33\end{array}$ & 405.68 & 5509.8 \\
\hline 355.24 & 4701.7 & 0.00 & 0.00 & 379.95 & 5080.9 & $\begin{array}{l}0.40 \\
1.40\end{array}$ & 0.05 & 405.75 & 5510.9 \\
\hline 355.55 & 4706.3 & 0.07 & 0.00 & 380.19 & 5084.9 & 42.27 & 1.56 & 406.05 & 5516.0 \\
\hline 355.85 & 4710.8 & 0.07 & 0.00 & 380.25 & 5085.9 & 11.00 & 0.41 & 406.34 & 5520.8 \\
\hline 356.15 & 4715.2 & 0.10 & 0.00 & 380.55 & 50909 & 14.04 & 0.52 & 406.65 & 5526.0 \\
\hline 356.45 & 4719.7 & 0.08 & 0.00 & 380.85 & 5095.9 & 44.65 & 1.65 & 406.95 & 5531.0 \\
\hline 356.69 & 4723.3 & 0.62 & 0.02 & 381.21 & 5101.9 & 45.24 & $\begin{array}{l}1.03 \\
1.67\end{array}$ & 407.18 & 5534.8 \\
\hline 357.05 & 4728.6 & 1.93 & 0.05 & 381.45 & 5105.9 & 44.80 & & 407.55 & $\begin{array}{l}5541.0 \\
554.0\end{array}$ \\
\hline 357.35 & 4733.1 & 0.06 & 0.00 & 382.05 & 5115.9 & 37.35 & & 407.84 & 5545.8 \\
\hline 357.65 & 4737.6 & 0.11 & 0.00 & 382.38 & 5121.4 & 44.97 & 1.66 & 408.15 & 5551.0 \\
\hline 358.19 & 4745.6 & 0.65 & 0.02 & 382.85 & 5129.2 & 45.69 & 173 & 408.45 & 5556.0 \\
\hline 358.24 & 4746.3 & 1.20 & 0.04 & 383.15 & 5134.2 & 41.81 & 1.58 & 408.68 & 5559.8 \\
\hline 358.55 & 4751.0 & 0.16 & 0.00 & 383.45 & 5139.2 & $\begin{array}{l}1.01 \\
46.52\end{array}$ & $\begin{array}{l}1.88 \\
1.88\end{array}$ & 408.75 & 5561.0 \\
\hline 358.85 & 4755.4 & 0.04 & 0.00 & 383.69 & 5143.2 & 25.67 & 0.95 & 409.05 & 5566.0 \\
\hline 359.15 & 4759.9 & 1.94 & 0.06 & 383.76 & 5144.4 & 22.60 & 0.72 & 409.34 & 5570.8 \\
\hline 359.45 & 4764.4 & 0.04 & 0.00 & 384.05 & 5149.2 & 9.03 & 0.30 & 409.95 & 5581.0 \\
\hline 359.69 & 47679 & 0.26 & 0.01 & 384.35 & 5154.2 & 0.20 & 0.01 & 410.18 & 5584.8 \\
\hline 360.05 & 4773.3 & 0.22 & 0.01 & 384.65 & $\begin{array}{l}5154.2 \\
5159.2\end{array}$ & 1.27 & 0.04 & 410.55 & 5591.0 \\
\hline 360.35 & 4777.8 & 0.09 & 0.00 & 384.95 & 5164.2 & 6.01 & 0.18 & 410.84 & 5595.8 \\
\hline 360.65 & 4782.2 & 1.68 & 0.06 & 385.19 & 5168.2 & 2.78 & 0.08 & 411.15 & 5601.0 \\
\hline 360.95 & 4786.5 & $\begin{array}{l}1.67 \\
\end{array}$ & 0.05 & 385.55 & 5174.2 & 0.34 & 0.01 & 411.35 & 5604.3 \\
\hline 361.19 & 4789.8 & 3.05 & 0.11 & 385.87 & 5179.5 & 0.55 & 0.02 & 411.65 & 5609.3 \\
\hline 361.24 & 4790.5 & 2.20 & 0.07 & 386.15 & 5184.2 & 0.83 & 0.02 & 411.95 & 5614.3 \\
\hline 361.55 & 4794.8 & 0.04 & 0.00 & $\begin{array}{l}380.15 \\
386.45\end{array}$ & $\begin{array}{l}5184.2 \\
5189.2\end{array}$ & $\begin{array}{l}0.83 \\
0.47\end{array}$ & 0.01 & 412.85 & 5629.3 \\
\hline 361.85 & 4799.0 & 1.15 & 0.04 & 386.69 & 5193.2 & 0.31 & 0.01 & 413.15 & 5634.3 \\
\hline 362.15 & 4803.2 & 0.27 & 0.01 & 386.76 & 5194.4 & 0.00 & 0.00 & 413.45 & 5639.3 \\
\hline 362.45 & 48073 & 0.95 & 0.04 & 387.05 & 5199.2 & 0.11 & 0.00 & 413.69 & 5643.3 \\
\hline 362.69 & 4810.7 & $\begin{array}{l}1.09 \\
\end{array}$ & 0.04 & 387.35 & 5204.2 & 0.11 & 0.00 & 414.05 & 5649.3 \\
\hline 363.05 & 4815.7 & 4.62 & 0.15 & 387.65 & 5209.2 & $\begin{array}{l}0.11 \\
0.15\end{array}$ & 0.00 & 414.35 & 5654.3 \\
\hline 363.35 & 4819.8 & 8.41 & 0.32 & 387.96 & 5214.3 & 0.06 & 0.00 & 414.65 & 5659.3 \\
\hline & 48240 & & & 38819 & 52182 & 031 & & 414.95 & 56643 \\
\hline
\end{tabular}


Table 1 (continued).

\begin{tabular}{|c|c|c|c|}
\hline $\begin{array}{l}\text { Composite } \\
\text { depth } \\
\text { (m) }\end{array}$ & $\begin{array}{l}\text { Age } \\
\text { (ka) }\end{array}$ & $\begin{array}{l}\mathrm{CaCO}_{3} \\
\text { (wt\%) }\end{array}$ & $\begin{array}{c}\mathrm{MAR} \\
\mathrm{CaCO}_{3} \\
\left(\mathrm{~g} / \mathrm{cm}^{2} \text { k.y. }\right)\end{array}$ \\
\hline 415.19 & 5668.3 & 1.18 & 0.03 \\
\hline 415.25 & 5669.3 & 2.20 & 0.05 \\
\hline 415.55 & 5674.3 & 1.34 & 0.04 \\
\hline 415.85 & 5679.3 & 3.17 & 0.10 \\
\hline 416.15 & 5684.3 & 16.94 & 0.54 \\
\hline 416.45 & 5689.4 & 0.13 & 0.00 \\
\hline 416.69 & 5693.4 & 0.05 & 0.00 \\
\hline 417.05 & 5699.4 & 0.09 & 0.00 \\
\hline 417.35 & 5704.4 & 0.06 & 0.00 \\
\hline 417.65 & 5709.4 & 0.13 & 0.00 \\
\hline 417.95 & 5714.4 & 0.00 & 0.00 \\
\hline 418.19 & 5718.4 & 4.43 & 0.15 \\
\hline 418.25 & 5719.4 & 5.60 & 0.19 \\
\hline 418.55 & 5724.4 & 1.34 & 0.04 \\
\hline 418.85 & 5729.4 & 0.04 & 0.00 \\
\hline 419.15 & 5734.4 & 0.06 & 0.00 \\
\hline 419.45 & 5739.4 & 0.00 & 0.00 \\
\hline 419.69 & 5743.4 & 0.24 & 0.01 \\
\hline 420.05 & 5749.4 & 15.19 & 0.49 \\
\hline 420.35 & 5754.4 & 25.50 & 0.97 \\
\hline 420.65 & 5759.4 & 0.57 & 0.02 \\
\hline 420.84 & 5762.5 & 0.00 & 0.00 \\
\hline 421.15 & 5767.7 & 0.15 & 0.00 \\
\hline 421.45 & 5772.7 & 2.01 & 0.06 \\
\hline 421.69 & 5776.7 & 3.41 & 0.10 \\
\hline 421.75 & 5777.7 & 2.80 & 0.09 \\
\hline 422.05 & 5782.7 & 1.13 & 0.03 \\
\hline 422.34 & 5787.6 & 0.58 & 0.02 \\
\hline 422.65 & 5792.7 & 0.19 & 0.01 \\
\hline 422.95 & 5797.7 & 0.06 & 0.00 \\
\hline 423.19 & 5801.7 & 0.00 & 0.00 \\
\hline 423.55 & 5807.7 & 0.10 & 0.00 \\
\hline 423.84 & 5812.6 & 0.10 & 0.00 \\
\hline 424.15 & 5817.7 & 0.05 & 0.00 \\
\hline 424.45 & 5822.7 & 0.00 & 0.00 \\
\hline 424.69 & 5826.7 & 0.00 & 0.00 \\
\hline 424.75 & 5827.7 & 0.00 & 0.00 \\
\hline 425.05 & 5832.7 & 0.07 & 0.00 \\
\hline 425.34 & 5837.6 & 0.65 & 0.02 \\
\hline 425.95 & 5847.7 & 1.06 & 0.03 \\
\hline 426.19 & 5851.7 & 0.07 & 0.00 \\
\hline 426.55 & 5857.8 & 1.24 & 0.04 \\
\hline 426.84 & 5862.6 & 0.11 & 0.00 \\
\hline 427.15 & 5867.8 & 0.07 & 0.00 \\
\hline 427.45 & 5872.8 & 0.29 & 0.01 \\
\hline 427.69 & 5876.8 & 0.06 & 0.00 \\
\hline 427.75 & 5877.8 & 0.00 & 0.00 \\
\hline 428.05 & 5882.8 & 0.06 & 0.00 \\
\hline 428.34 & 5887.6 & 0.13 & 0.00 \\
\hline 428.65 & 5892.8 & 0.63 & 0.02 \\
\hline 428.95 & 5897,8 & 0.28 & 0.01 \\
\hline 429.19 & 5901.8 & 0.35 & 0.01 \\
\hline 429.55 & 5907.8 & 0.27 & 0.01 \\
\hline 429.84 & 5912.6 & 0.32 & 0.01 \\
\hline 430.15 & 5917.8 & 0.00 & 0.00 \\
\hline
\end{tabular}

Site 882

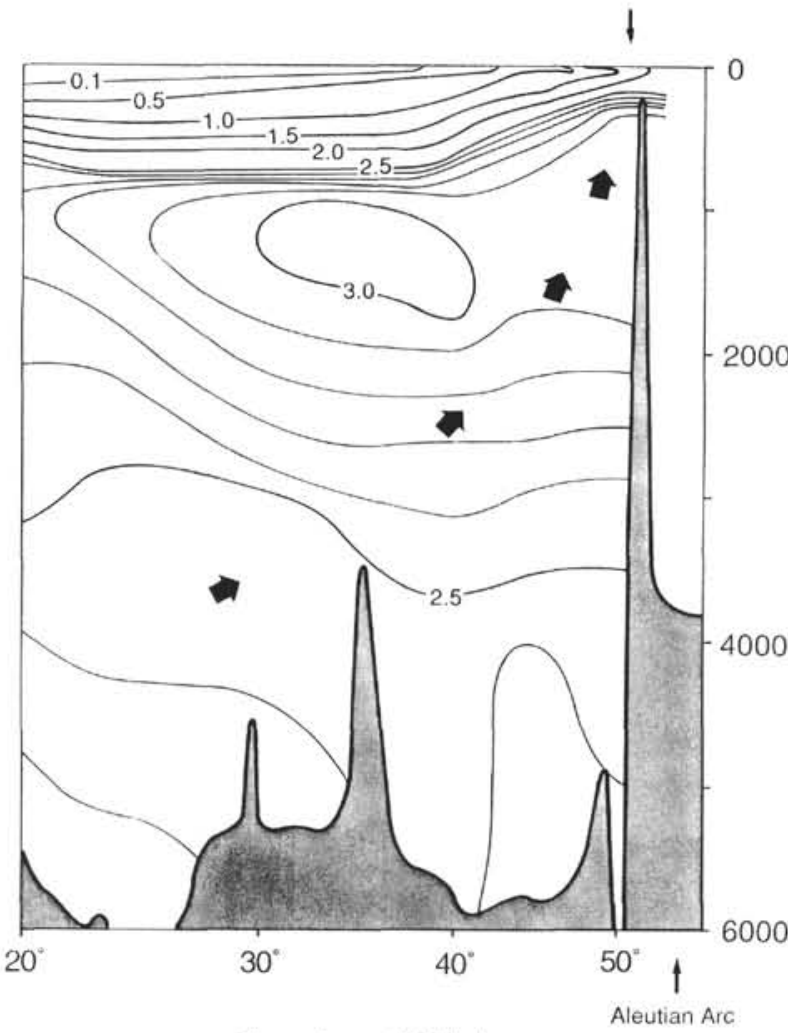

Phosphate $(\mu \mathrm{M} / \mathrm{kg})$

Figure 3. Vertical distribution of phosphate $(\mu \mathrm{M} / \mathrm{kg})$ from GEOSECS Stations 214-219 and 227 obtained from the GEOSOCS Pacific expedition 1973-1974 in the western Pacific between $20^{\circ}$ and $50^{\circ} \mathrm{N}$ (Craig et al., 1981). Note the strong upward diffusion of North Pacific deep water (arrows) indicated by phosphate as example of nutrient distribution in the northwest Pacific. 
Table 2. Biogenic opal and siliciclastic fraction $>2 \mu \mathrm{m}$ data from Site $\mathbf{8 8 2}$.

\begin{tabular}{|c|c|c|c|c|c|c|c|c|c|c|}
\hline $\begin{array}{l}\text { Composite } \\
\text { depth } \\
\text { (m) }\end{array}$ & $\begin{array}{l}\text { Age } \\
\text { (ka) }\end{array}$ & $\begin{array}{c}\text { Opal } \\
\text { (wt\%) }\end{array}$ & $\begin{array}{l}\text { MAR opal } \\
\left(\mathrm{g} / \mathrm{cm}^{2} \mathrm{k} \cdot \mathrm{y} .\right)\end{array}$ & $\begin{array}{l}\text { Siliciclastic } \\
\text { fraction } \\
>2 \mu \mathrm{m} \\
(\mathrm{wt} \%)\end{array}$ & $\begin{array}{l}\text { MAR silici- } \\
\text { clastic fraction } \\
>2 \mu \mathrm{m} \\
\left(\mathrm{g} / \mathrm{cm}^{2} \mathrm{k} . \mathrm{y} .\right)\end{array}$ & $\begin{array}{l}\text { Composite } \\
\text { depth } \\
\text { (m) }\end{array}$ & $\begin{array}{l}\text { Age } \\
\text { (ka) }\end{array}$ & $\begin{array}{c}\text { Opal } \\
\text { (wt\%) }\end{array}$ & $\begin{array}{l}\text { MAR opal } \\
\left(\mathrm{g} / \mathrm{cm}^{2} \text { k.y. }\right)\end{array}$ & $\begin{array}{l}\text { Siliciclastic } \\
\text { fraction } \\
>2 \mu \mathrm{m} \\
(\mathrm{wt} \%)\end{array}$ \\
\hline 0.30 & 4.9 & 10.73 & 0.37 & 32.23 & 1.12 & 28.87 & 566.0 & 6.75 & 0.11 & 33.52 \\
\hline 0.60 & 9.8 & 22.05 & 0.72 & 18.21 & 0.59 & 29.12 & 579.1 & 24.06 & 0.21 & 20.72 \\
\hline 0.90 & 14.7 & 3.09 & 0.10 & 26.55 & 0.85 & 29.36 & 590.8 & 12.30 & 0.17 & 29.60 \\
\hline 1.20 & 20.1 & 4.01 & 0.13 & 29.67 & 0.98 & 29.55 & 598.5 & 11.64 & 0.13 & 21.87 \\
\hline 1.55 & 26.4 & 4.54 & 0.14 & 28.40 & 0.88 & 29.80 & 608.6 & 6.28 & 0.12 & 31.63 \\
\hline 1.85 & 31.9 & 5.00 & 0.16 & 30.19 & 0.94 & 30.04 & 613.0 & 10.13 & 0.27 & 28.84 \\
\hline 2.10 & 36.4 & 4.39 & 0.14 & 33.63 & 1.08 & 30.28 & 617.1 & 9.81 & 0.27 & 33.64 \\
\hline 2.36 & 41.1 & 5.70 & 0.17 & 53.89 & 1.59 & 30.53 & 621.5 & 32.40 & 0.81 & 20.80 \\
\hline 2.66 & 46.6 & 11.10 & 0.40 & 52.66 & 1.89 & 30.77 & 625.6 & 29.21 & 0.70 & 16.53 \\
\hline 2.96 & 52.0 & 15.84 & 0.46 & 44.96 & 1.31 & 31.00 & 629.6 & 19.87 & 0.53 & 31.46 \\
\hline 3.20 & 56.4 & 12.75 & 0.42 & 52.53 & 1.74 & 31.25 & 635.0 & 5.02 & 0.13 & 35.98 \\
\hline 3.56 & 62.9 & 2.85 & 0.09 & 44.33 & 1.42 & 31.50 & 640.8 & 20.52 & 0.46 & 28.14 \\
\hline 3.86 & 68.4 & 1.21 & 0.05 & 13.83 & 0.54 & 31.74 & 645.2 & 8.53 & 0.25 & 26.75 \\
\hline 4.16 & 73.1 & 36.01 & 0.96 & 32.52 & 0.87 & 32.20 & 652.9 & 6.86 & 0.22 & 30.02 \\
\hline 4.46 & 77.4 & 17.03 & 0.65 & 30.60 & 1.17 & 32.49 & 657.8 & 4.95 & 0.19 & 39.32 \\
\hline 4.70 & 80.9 & 16.86 & 0.60 & 45.72 & 1.62 & 32.73 & 661.8 & 5.57 & 0.23 & 45.78 \\
\hline 5.36 & 90.5 & 12.61 & 0.55 & 49.06 & 2.13 & 33.08 & 667.6 & 9.31 & 0.32 & 27.92 \\
\hline 5.66 & 94.9 & 46.22 & 1.17 & 22.19 & 0.56 & 33.37 & 672.4 & 4.61 & 0.15 & 29.20 \\
\hline 5.96 & 99.2 & 51.16 & 1.30 & 22.10 & 0.56 & 33.67 & 677.4 & 3.50 & 0.14 & 42.08 \\
\hline 6.20 & 102.7 & 44.53 & 1.00 & 31.40 & 0.71 & 33.96 & 682.1 & 19.88 & 0.68 & 35.90 \\
\hline 6.86 & 113.4 & 3.02 & 0.10 & 49.98 & 1.72 & 34.20 & 685.7 & 6.70 & 0.21 & 51.55 \\
\hline 7.16 & 122.0 & 21.36 & 0.36 & 34.15 & 0.58 & 34.55 & 691.0 & 35.52 & 1.22 & 23.7 \\
\hline 7.46 & 132.5 & 12.33 & 0.23 & 11.69 & 0.22 & 34.84 & 695.4 & 36.54 & 0.89 & 17.73 \\
\hline 7.70 & 139.1 & 8.91 & 0.23 & 47.24 & 1.21 & 35.14 & 700.0 & 16.67 & 0.63 & 24.21 \\
\hline 8.06 & 146.1 & 8.42 & 0.24 & 38.74 & 1.11 & 35.67 & 708.0 & 40.22 & 1.04 & 4.10 \\
\hline 8.36 & 151.9 & 7.15 & 0.23 & 41.31 & 1.36 & 36.31 & 717.7 & 26.36 & 0.74 & 21.69 \\
\hline 8.66 & 157.7 & 5.49 & 0.24 & 50.37 & 2.16 & 36.61 & 722.2 & 6.13 & 0.21 & 52.03 \\
\hline 9.20 & 164.4 & 9.00 & 0.39 & 40.02 & 1.73 & 36.91 & 729.9 & 11.35 & 0.26 & 29.75 \\
\hline 9.49 & 167.4 & 9.64 & 0.40 & 28.29 & 1.18 & 37.14 & 736.3 & 15.74 & 0.32 & 45.56 \\
\hline 9.69 & 169.4 & 4.84 & 0.27 & 36.06 & 2.03 & 37.50 & 746.3 & 13.53 & 0.28 & 26.94 \\
\hline 9.93 & 171.9 & 5.14 & 0.31 & 41.25 & 2.45 & 37.78 & 754.1 & 3.81 & 0.09 & 27.50 \\
\hline 10.17 & 174.4 & 8.45 & 0.41 & 37.56 & 1.81 & 38.08 & 762.0 & 10.75 & 0.25 & 31.69 \\
\hline 10.45 & 177.3 & 4.27 & 0.21 & 33.20 & 1.65 & 38.38 & 769.3 & 14.02 & 0.33 & 33.11 \\
\hline 10.68 & 179.7 & 3.22 & 0.19 & 40.05 & 2.38 & 38.61 & 775.0 & 5.46 & 0.19 & 41.21 \\
\hline 10.95 & 182.5 & 10.57 & 0.59 & 40.34 & 2.23 & 38.97 & 781.5 & 44.75 & 1.34 & 18.21 \\
\hline 11.17 & 184.7 & 2.45 & 0.15 & 27.06 & 1.68 & 39.23 & 786.0 & 16.60 & 0.28 & 14.03 \\
\hline 11.42 & 187.9 & 5.38 & 0.18 & 65.86 & 2.19 & 39.56 & 800.2 & 3.80 & 0.06 & 29.48 \\
\hline 11.64 & 194.3 & 17.21 & 0.32 & 32.01 & 0.60 & 39.85 & 812.7 & 6.33 & 0.14 & 36.89 \\
\hline 11.94 & 203.2 & 13.22 & 0.24 & 36.90 & 0.66 & 40.09 & 818.0 & 5.45 & 0.19 & 36.86 \\
\hline 12.23 & 211.7 & 4.19 & 0.09 & 44.10 & 0.97 & 40.44 & 824.6 & 15.65 & 0.29 & 38.61 \\
\hline 12.41 & 217.0 & 17.35 & 0.23 & 29.82 & 0.40 & 40.73 & 833.5 & 12.40 & 0.26 & 36.95 \\
\hline 12.52 & 220.5 & 45.05 & 0.43 & 21.12 & 0.20 & 40.90 & 838.9 & 13.72 & 0.31 & 40.08 \\
\hline 12.81 & 230.7 & 24.32 & 0.43 & 30.41 & 0.53 & 41.44 & 847.2 & 7.60 & 0.33 & 34.66 \\
\hline 13.10 & 237.8 & 28.75 & 0.61 & 25.25 & 0.53 & 41.69 & 850.1 & 4.86 & 0.24 & 37.22 \\
\hline 13.40 & 245.1 & 27.94 & 0.44 & 26.40 & 0.41 & 42.04 & 855.0 & 2.67 & 0.11 & 86.35 \\
\hline 13.65 & 251.2 & 4.51 & 0.14 & 55.92 & 1.76 & 42.28 & 859.8 & 2.51 & 0.08 & 37.89 \\
\hline 13.97 & 256.9 & 34.22 & 0.99 & 49.43 & 1.42 & 42.53 & 864.8 & 3.63 & 0.11 & 30.52 \\
\hline 14.27 & 262.2 & 16.55 & 0.42 & 35.13 & 0.89 & 42.83 & 870.8 & 3.83 & 0.12 & 33.14 \\
\hline 14.56 & 267.4 & 9.13 & 0.36 & 42.81 & 1.68 & 43.07 & 875.1 & 2.83 & 0.13 & 43.56 \\
\hline 14.86 & 272.7 & 5.09 & 0.19 & 90.81 & 3.41 & 43.44 & 879.9 & 14.51 & 0.68 & 41.63 \\
\hline 15.07 & 276.4 & 2.49 & 0.10 & 40.26 & 1.59 & 43.68 & 883.1 & 46.27 & 1.04 & 17.13 \\
\hline 15.43 & 282.5 & 3.99 & 0.14 & 53.74 & 1.89 & 44.04 & 890.2 & 15.94 & 0.45 & 51.63 \\
\hline 15.67 & 286.6 & 5.61 & 0.23 & 63.58 & 2.61 & 44.34 & 896.4 & 11.12 & 0.35 & 41.29 \\
\hline 16.01 & 292.3 & 15.34 & 0.55 & 32.59 & 1.18 & 44.65 & 902.4 & 3.79 & 0.14 & 62.02 \\
\hline 16.31 & 297.4 & 7.77 & 0.26 & 42.02 & 1.39 & 44.94 & 908.1 & 77.85 & 1.19 & 7.44 \\
\hline 16.53 & 301.2 & 3.24 & 0.12 & 84.74 & 3.04 & 45.18 & 912.8 & 3.84 & 0.16 & 52.85 \\
\hline 16.89 & 309.9 & 35.02 & 0.75 & 22.87 & 0.49 & 45.54 & 919.9 & 64.98 & 1.04 & 4.30 \\
\hline 17.18 & 316.9 & 11.45 & 0.26 & 33.09 & 0.75 & 45.84 & 925.8 & 26.16 & 0.72 & 30.96 \\
\hline 17.47 & 324.0 & 48.79 & 1.11 & 24.75 & 0.56 & 46.15 & 931.7 & 39.64 & 1.04 & 22.21 \\
\hline 17.77 & 331.2 & 6.50 & 0.18 & 50.53 & 1.41 & 46.44 & 937.2 & 5.12 & 0.18 & 48.16 \\
\hline 17.98 & 336.3 & 34.51 & 0.62 & 29.45 & 0.53 & 46.68 & 941.8 & 4.17 & 0.19 & 42.23 \\
\hline 18.34 & 344.7 & 3.97 & 0.14 & 81.81 & 2.92 & 47.33 & 950.7 & 58.44 & 2.27 & 16.73 \\
\hline 18.63 & 349.4 & 3.89 & 0.16 & 31.49 & 1.31 & 47.65 & 954.4 & 60.20 & 1.59 & 15.78 \\
\hline 18.92 & 354.1 & 9.83 & 0.32 & 34.50 & 1.13 & 47.94 & 958.8 & 62.72 & 0.05 & 11.45 \\
\hline 19.22 & 358.9 & 12.79 & 0.42 & 43.05 & 1.40 & 48.18 & 964.8 & 13.15 & 0.29 & 34.73 \\
\hline 19.44 & 362.3 & 6.42 & 0.37 & 49.07 & 2.86 & 48.54 & 973.9 & 47.08 & 1.01 & 16.03 \\
\hline 20.07 & 369.1 & 0.00 & 0.00 & 50.53 & 2.10 & 48.83 & 981.7 & 26.94 & 0.47 & 32.38 \\
\hline 20.34 & 372.0 & 9.68 & 0.23 & 31.28 & 0.74 & 49.25 & 994.1 & 47.54 & 0.69 & 16.73 \\
\hline 20.56 & 379.8 & 10.74 & 0.16 & 29.83 & 0.45 & 49.38 & 997.9 & 18.81 & 0.30 & 19.55 \\
\hline 20.80 & 388.2 & 10.50 & 0.15 & 40.39 & 0.59 & 49.62 & 1003.8 & 15.36 & 0.35 & 24.95 \\
\hline 21.05 & 397.0 & 9.57 & 0.21 & 38.40 & 0.85 & 49.91 & 1010.3 & 15.54 & 0.36 & 25.18 \\
\hline 21.29 & 401.8 & 5.03 & 0.14 & 38.67 & 1.10 & 50.21 & 1017.0 & 6.03 & 0.17 & 34.68 \\
\hline 21.87 & 413.2 & 7.88 & 0.25 & 27.05 & 0.84 & 50.45 & 1022.3 & 3.90 & 0.10 & 27.82 \\
\hline 22.14 & 418.5 & 3.24 & 0.12 & 65.21 & 2.47 & 50.74 & 1028.8 & 13.23 & 0.32 & 28.68 \\
\hline 22.73 & 429.8 & 4.65 & 0.17 & 29.19 & 1.09 & 50.99 & 1034.4 & 16.46 & 0.39 & 28.64 \\
\hline 23.03 & 435.6 & 3.51 & 0.12 & 30.44 & 1.06 & 51.28 & 1040.9 & 42.92 & 0.70 & 15.35 \\
\hline 23.62 & 446.8 & 12.53 & 0.33 & 28.29 & 0.74 & 51.52 & 1046.2 & 39.72 & 0.58 & 16.36 \\
\hline 24.81 & 475.3 & 4.49 & 0.12 & 54.55 & 1.43 & 51.81 & 1051.6 & 32.33 & 0.73 & 20.11 \\
\hline 25.08 & 479.1 & 34.25 & 1.01 & 18.20 & 0.54 & 52.03 & 1054.5 & 8.13 & 0.38 & 38.12 \\
\hline 25.67 & 487.6 & 25.60 & 0.89 & 29.55 & 1.03 & 52.05 & 1054.7 & 1.00 & 0.04 & 46.99 \\
\hline 25.97 & 491.9 & 16.23 & 0.75 & 46.16 & 2.12 & 52.47 & 1060.2 & 3.92 & 0.19 & 37.99 \\
\hline 26.28 & 496.3 & 17.48 & 0.59 & 24.25 & 0.81 & 52.75 & 1064.8 & 18.10 & 0.61 & 35.19 \\
\hline 26.55 & 500.3 & 38.49 & 1.32 & 15.68 & 0.54 & 52.98 & 1068.8 & 9.28 & 0.31 & 65.50 \\
\hline 26.65 & 501.7 & 42.83 & 1.46 & 15.01 & 0.51 & 53.33 & 1075.0 & 11.51 & 0.41 & 19.28 \\
\hline 27.44 & 513.3 & 12.40 & 0.37 & 33.49 & 0.99 & 53.61 & 1080.0 & 11.28 & 0.33 & 30.09 \\
\hline 27.75 & 523.8 & 10.11 & 0.15 & 38.20 & 0.56 & 53.91 & 1087.7 & 6.70 & 0.15 & 61.95 \\
\hline 28.00 & 535.3 & 19.92 & 0.31 & 29.67 & 0.47 & 54.19 & 1096.0 & 13.82 & 0.30 & 39.89 \\
\hline
\end{tabular}


Table 2 (continued).

\begin{tabular}{|c|c|c|c|c|c|c|c|c|c|c|}
\hline $\begin{array}{l}\text { Composite } \\
\text { depth } \\
\text { (m) }\end{array}$ & $\begin{array}{l}\text { Age } \\
\text { (ka) }\end{array}$ & $\begin{array}{l}\text { Opal } \\
\text { (wt } \%)\end{array}$ & $\begin{array}{l}\text { MAR opal } \\
\left(\mathrm{g} / \mathrm{cm}^{2} \text { k.y. }\right)\end{array}$ & $\begin{array}{l}\text { Siliciclastic } \\
\text { fraction } \\
>2 \mu \mathrm{m} \\
(\mathrm{wt} \%)\end{array}$ & $\begin{array}{l}\text { MAR silici- } \\
\text { clastic fraction } \\
>2 \mu \mathrm{m} \\
\left(\mathrm{g} / \mathrm{cm}^{2} \mathrm{k} . \mathrm{y} .\right)\end{array}$ & $\begin{array}{l}\text { Composite } \\
\text { depth } \\
\text { (m) }\end{array}$ & $\begin{array}{l}\text { Age } \\
(\mathrm{ka})\end{array}$ & $\begin{array}{l}\text { Opal } \\
\text { (wt\%) }\end{array}$ & $\begin{array}{l}\text { MAR opal } \\
\left(\mathrm{g} / \mathrm{cm}^{2} \text { k.y. }\right)\end{array}$ & $\begin{array}{c}\text { Siliciclastic } \\
\text { fraction } \\
>2 \mu \mathrm{m} \\
\text { (wt\%) }\end{array}$ \\
\hline 54.42 & 1102.8 & 12.33 & 0.27 & 37.70 & 0.83 & 80.29 & 1738.9 & 6.08 & 0.20 & 61.07 \\
\hline 55.05 & 1118.6 & 17.44 & 0.35 & 19.11 & 0.38 & 80.67 & 1745.8 & 14.10 & 0.50 & 56.32 \\
\hline 55.36 & 1126.3 & 23.02 & 0.43 & 20.54 & 0.38 & 80.76 & 1747.5 & 1.99 & 0.08 & 88.57 \\
\hline 55.86 & 1140.9 & 8.84 & 0.15 & 32.65 & 0.57 & 80.86 & 1749.3 & 2.40 & 0.08 & 81.28 \\
\hline 56.21 & 1156.3 & 12.67 & 0.18 & 35.90 & 0.52 & 80.97 & 1751.4 & 2.51 & 0.10 & 53.71 \\
\hline 56.49 & 1168.7 & 7.40 & 0.09 & 44.70 & 0.57 & 81.04 & 1752.6 & 4.57 & 0.16 & 41.64 \\
\hline 56.80 & 1180.2 & 9.62 & 0.21 & 34.94 & 0.78 & 81.37 & 1758.7 & 20.73 & 0.53 & 27.24 \\
\hline 57.08 & 1186.4 & 26.00 & 0.68 & 21.76 & 0.57 & 81.47 & 1762.1 & 20.14 & 0.23 & 25.57 \\
\hline 57.31 & 1191.5 & 37.77 & 0.81 & 15.91 & 0.34 & 81.56 & 1768.4 & 9.30 & 0.09 & 32.34 \\
\hline 57.65 & 1198.9 & 30.50 & 0.72 & 14.80 & 0.35 & 81.75 & 1781.6 & 3.05 & 0.03 & 62.63 \\
\hline 57.93 & 1205.1 & 24.81 & 0.64 & 15.06 & 0.39 & 81.94 & 1794.8 & 3.86 & 0.04 & 76.20 \\
\hline 58.23 & 1210.4 & 21.78 & 0.71 & 28.08 & 0.92 & 82.03 & 1800.3 & 5.24 & 0.07 & 61.49 \\
\hline 58.52 & 1215.3 & 18.43 & 0.60 & 21.51 & 0.70 & 82.16 & 1806.2 & 4.63 & 0.05 & 21.74 \\
\hline 58.75 & 1219.3 & 20.78 & 0.64 & 24.72 & 0.76 & 82.45 & 1825.9 & 10.98 & 0.08 & 29.33 \\
\hline 59.09 & 1225.4 & 39.31 & 0.84 & 17.55 & 0.37 & 82.68 & 1843.6 & 5.26 & 0.05 & 42.06 \\
\hline 59.37 & 1231.9 & 17.08 & 0.43 & 32.45 & 0.81 & 83.03 & 1873.6 & 4.24 & 0.04 & 71.05 \\
\hline 59.67 & 1238.9 & 41.11 & 0.56 & 19.04 & 0.26 & 83.61 & 1917.8 & 2.30 & 0.02 & 84.81 \\
\hline 60.19 & 1251.0 & 42.34 & 0.81 & 22.37 & 0.43 & 83.90 & 1934.8 & 8.17 & 0.10 & 74.99 \\
\hline 60.53 & 1257.8 & 35.49 & 0.81 & 21.15 & 0.48 & 84.14 & 1947.7 & 11.76 & 0.21 & 72.89 \\
\hline 60.70 & 1260.8 & 29.33 & 0.46 & 21.14 & 0.33 & 84.49 & 1955.5 & 4.82 & 0.16 & 83.68 \\
\hline 60.91 & 1264.6 & 24.56 & 0.59 & 29.35 & 0.71 & 84.78 & 1962.0 & 13.17 & 0.41 & 71.17 \\
\hline 61.09 & 1267.8 & 19.06 & 0.48 & 20.56 & 0.51 & 85.07 & 1968.5 & 5.36 & 0.19 & 53.91 \\
\hline 61.23 & 1270.3 & 12.87 & 0.30 & 24.80 & 0.57 & 85.34 & 1974.6 & 4.40 & 0.13 & 83.56 \\
\hline 61.43 & 1274.7 & 9.73 & 0.21 & 42.02 & 0.89 & 85.59 & 1981.4 & 5.29 & 0.13 & 78.89 \\
\hline 61.62 & 1281.0 & 32.56 & 0.50 & 16.84 & 0.26 & 85.94 & 1991.1 & 8.26 & 0.19 & 77.38 \\
\hline 61.71 & 1284.0 & 58.87 & 0.61 & 6.52 & 0.07 & 86.23 & 1999.2 & 5.50 & 0.11 & 43.53 \\
\hline 61.88 & 1289.7 & 6.65 & 0.12 & 35.90 & 0.65 & 86.81 & 2017.2 & 7.57 & 0.15 & 37.18 \\
\hline 62.13 & 1298.0 & 16.89 & 0.31 & 33.91 & 0.62 & 87.09 & 2029.2 & 7.72 & 0.10 & 28.63 \\
\hline 62.36 & 1305.7 & 5.09 & 0.13 & 39.03 & 1.01 & 87.40 & 2042.5 & 5.25 & 0.08 & 35.27 \\
\hline $62.7 \downarrow$ & 1315.2 & 9.07 & 0.22 & 32.93 & 0.81 & 87.69 & 2054.2 & 7.43 & 0.16 & 33.86 \\
\hline 63.00 & 1322.8 & 5.99 & 0.14 & 32.72 & 0.75 & 87.98 & 2063.2 & 17.58 & 0.38 & 29.58 \\
\hline 63.31 & 1331.0 & 7.65 & 0.18 & 37.39 & 0.88 & 88.09 & 2066.7 & 7.88 & 0.18 & 47.11 \\
\hline 63.59 & 1338.1 & 4.94 & 0.15 & 37.71 & 1.15 & 88.46 & 2078.2 & 6.63 & 0.13 & 50.54 \\
\hline 63.82 & 1343.3 & 25.18 & 0.82 & 35.89 & 1.16 & 88.85 & 2092.8 & 6.80 & 0.10 & 43.44 \\
\hline 64.14 & 1350.6 & 3.85 & 0.12 & 59.85 & 1.90 & 89.14 & 2107.0 & 10.51 & 0.16 & 26.82 \\
\hline 64.46 & 1357.8 & 9.41 & 0.14 & 46.33 & 0.67 & 89.43 & 2121.2 & 5.84 & 0.09 & 47.60 \\
\hline 65.04 & 1385.6 & 2.22 & 0.04 & 35.46 & 0.61 & 89.94 & 2143.4 & 9.53 & 0.15 & 35.63 \\
\hline 65.27 & 1392.8 & 7.64 & 0.19 & 38.86 & 0.99 & 90.31 & 2158.4 & 10.70 & 0.18 & 37.71 \\
\hline 65.62 & 1401.2 & 4.73 & 0.12 & 30.12 & 0.75 & 90.75 & 2173.2 & 8.61 & 0.18 & 32.22 \\
\hline 65.91 & 1408.1 & 3.96 & 0.10 & 28.26 & 0.70 & 90.77 & 2173.5 & 13.18 & 0.52 & 27.81 \\
\hline 66.20 & 1415.1 & 33.08 & 1.01 & 51.67 & 1.58 & 90.97 & 2176.9 & 12.17 & 0.47 & 28.27 \\
\hline 66.49 & 1422.0 & $\begin{array}{r}2.49 \\
\end{array}$ & 0.07 & 58.86 & 1.62 & 91.37 & 2183.7 & 6.81 & 0.22 & 32.97 \\
\hline 66.73 & 1429.2 & 6.08 & 0.13 & 35.85 & 0.74 & 91.97 & 2194.2 & 20.27 & 0.73 & 36.61 \\
\hline 67.08 & 1441.5 & 9.68 & 0.18 & 27.15 & 0.49 & 92.22 & 2198.7 & 40.74 & 1.08 & 14.11 \\
\hline 67.37 & 1452.4 & 9.56 & 0.15 & 32.11 & 0.50 & 92.57 & 2205.0 & 27.42 & 0.88 & 22.00 \\
\hline 67.66 & 1460.3 & 7.81 & 0.17 & 41.78 & 0.91 & 92.87 & 2210.4 & 29.18 & 0.93 & 16.94 \\
\hline 67.95 & 1468.6 & 19.44 & 0.41 & 30.43 & 0.65 & 93.09 & 2214.4 & 28.55 & 1.01 & 16.18 \\
\hline 68.18 & 1475.4 & 19.23 & 0.30 & 29.11 & 0.45 & 93.44 & 2220.0 & 27.29 & 0.84 & 18.21 \\
\hline 68.53 & 1487.7 & 9.00 & 0.14 & 18.22 & 0.29 & 93.72 & 2224.2 & 52.55 & 1.19 & 8.10 \\
\hline 68.82 & 1500.5 & 8.86 & 0.17 & 40.86 & 0.77 & 94.01 & 2228.7 & 18.85 & 0.55 & 17.20 \\
\hline 69.11 & 1509.3 & 5.06 & 0.11 & 34.50 & 0.77 & 94.30 & 2233.1 & 40.94 & 0.96 & 13.61 \\
\hline 69.41 & 1516.8 & 25.56 & 0.68 & 31.59 & 0.84 & 94.49 & 2236.0 & 38.98 & 1.19 & 17.16 \\
\hline 69.64 & 1522.5 & 49.94 & 0.94 & 18.28 & 0.35 & 94.88 & 2241.9 & 36.43 & 1.37 & 23.93 \\
\hline 69.99 & 1531.3 & 66.47 & 0.97 & 8.96 & 0.13 & 95.17 & 2246.4 & 17.73 & 0.70 & 22.28 \\
\hline 70.29 & 1538.5 & 35.93 & 0.82 & 4.83 & 0.11 & 95.46 & 2250.8 & 10.58 & 0.31 & 26.53 \\
\hline 70.41 & 1540.7 & 59.45 & 1.03 & 21.95 & 0.38 & 95.65 & 2255.9 & 8.05 & 0.14 & 35.99 \\
\hline 70.65 & 1545.1 & 52.16 & 1.29 & 15.78 & 0.39 & 95.98 & 2267.5 & 21.94 & 0.31 & 24.13 \\
\hline 70.84 & 1548.5 & 34.84 & 0.95 & 15.05 & 0.41 & 96.32 & 2279.4 & 43.90 & 0.58 & 9.57 \\
\hline 71.02 & 1551.7 & 18.06 & 0.53 & 38.22 & 1.13 & 96.61 & 2289.6 & 14.22 & 0.30 & 19.60 \\
\hline 71.22 & 1555.3 & 9.39 & 0.34 & 18.53 & 0.67 & 96.90 & 2299.8 & 43.24 & 0.70 & 15.02 \\
\hline 71.41 & 1558.8 & 7.27 & 0.29 & 24.03 & 0.95 & 97.19 & 2310.0 & 25.62 & 0.27 & 14.86 \\
\hline 71.60 & 1562.2 & 13.34 & 0.42 & 59.58 & 1.86 & 97.42 & 2323.2 & 28.07 & 0.24 & 16.34 \\
\hline 71.79 & 1565.6 & 22.95 & 0.43 & 26.34 & 0.50 & 97.76 & 2344.0 & 11.34 & 0.11 & 28.22 \\
\hline 71.98 & 1572.9 & 22.21 & 0.37 & 33.55 & 0.56 & 98.05 & 2361.8 & 13.16 & 0.14 & 20.17 \\
\hline 72.17 & 1580.7 & 24.65 & 0.45 & 26.45 & 0.49 & 98.36 & 2376.0 & 7.44 & 0.16 & 18.81 \\
\hline 72.36 & 1587.1 & 36.38 & 0.71 & 22.96 & 0.44 & 98.63 & 2380.8 & 12.38 & 0.37 & 21.74 \\
\hline 72.45 & 1588.6 & 9.69 & 0.35 & 29.08 & 1.05 & 98.86 & 2384.9 & 9.33 & 0.36 & 37.98 \\
\hline 72.79 & 1594.5 & 5.17 & 0.19 & 24.63 & 0.93 & 99.21 & 2391.1 & 16.49 & 0.56 & 17.62 \\
\hline 73.08 & 1599.6 & 7.92 & 0.25 & 29.31 & 0.91 & 99.50 & 2396.2 & 10.93 & 0.41 & 23.42 \\
\hline 73.37 & 1604.7 & 11.38 & 0.41 & 30.68 & 1.10 & 99.78 & 2401.2 & 11.06 & 0.38 & 27.21 \\
\hline 73.66 & 1609.0 & 6.54 & 0.30 & 37.99 & 1.77 & 100.07 & 2406.4 & 7.25 & 0.25 & 24.64 \\
\hline 73.89 & 1612.4 & 6.33 & 0.27 & 27.33 & 1.17 & 100.30 & 2410.5 & 5.37 & 0.18 & 31.77 \\
\hline 74.53 & 1621.8 & 6.07 & 0.27 & 40.25 & 1.76 & 100.65 & 2418.6 & 9.63 & 0.27 & 27.5 \\
\hline 74.84 & 1627.5 & 21.71 & 0.54 & 22.71 & 0.56 & 100.97 & 2426.2 & 14.11 & 0.31 & 22.65 \\
\hline 75.11 & 1635.0 & 13.08 & 0.33 & 34.70 & 0.89 & 101.12 & 2430.3 & 9.14 & 0.16 & 37.75 \\
\hline 75.69 & 1649.5 & 8.64 & 0.23 & 37.63 & 1.01 & 101.50 & 2446.5 & 6.98 & 0.11 & 48.62 \\
\hline 75.98 & 1655.4 & 8.21 & 0.25 & 38.73 & 1.20 & 101.88 & 2462.3 & 19.72 & 0.25 & 19.20 \\
\hline 76.28 & 1661.5 & 4.10 & 0.13 & 37.25 & 1.21 & 102.03 & 2468.6 & 38.77 & 0.46 & 13.71 \\
\hline 76.55 & 1667.1 & 8.21 & 0.24 & 46.09 & 1.34 & 102.22 & 2476.5 & 26.81 & 0.28 & 19.03 \\
\hline 76.79 & 1672.0 & 3.35 & 0.13 & 37.42 & 1.44 & 102.41 & 2484.4 & 13.11 & 0.17 & 37.20 \\
\hline 77.13 & 1677.6 & 8.41 & 0.36 & 62.94 & 2.70 & 102.50 & 2488.2 & 7.11 & 0.10 & 51.83 \\
\hline 77.73 & 1686.6 & 5.44 & 0.24 & 63.18 & 2.77 & 102.75 & 2500.9 & 11.81 & 0.14 & 29.69 \\
\hline 78.00 & 1690.7 & 9.22 & 0.49 & 64.15 & 3.43 & 103.05 & 2516.4 & 18.29 & 0.26 & 27.44 \\
\hline 78.23 & 1694.1 & 8.36 & 0.24 & 28.83 & 0.83 & 103.28 & 2528.3 & 9.58 & 0.16 & 34.85 \\
\hline 78.87 & 1710.1 & 4.05 & 0.13 & 54.44 & 1.73 & 103.63 & 2541.8 & 21.91 & 0.39 & 20.11 \\
\hline 79.68 & 1727.5 & 1.41 & 0.05 & 90.54 & 3.30 & 103.92 & 2551.3 & 19.56 & 0.33 & 22.08 \\
\hline 80.02 & 1733.9 & 3.21 & 0.12 & 38.44 & 1.47 & 104.21 & 2560.8 & 22.69 & 0.35 & 27.14 \\
\hline
\end{tabular}


Table 2 (continued).

\begin{tabular}{|c|c|c|c|c|c|c|c|c|c|c|}
\hline $\begin{array}{l}\text { Composite } \\
\text { depth } \\
\text { (m) }\end{array}$ & $\begin{array}{l}\text { Age } \\
\text { (ka) }\end{array}$ & $\begin{array}{l}\text { Opal } \\
(w t \%)\end{array}$ & $\begin{array}{l}\text { MAR opal } \\
\left(\mathrm{g} / \mathrm{cm}^{2} \text { k.y. }\right)\end{array}$ & $\begin{array}{c}\text { Siliciclastic } \\
\text { fraction } \\
>2 \mu \mathrm{m} \\
(\mathrm{wt} \%)\end{array}$ & $\begin{array}{l}\text { MAR silici- } \\
\text { clastic fraction } \\
>2 \mu \mathrm{m} \\
\left(\mathrm{g} / \mathrm{cm}^{2} \mathrm{k} . \mathrm{y} .\right)\end{array}$ & $\begin{array}{l}\text { Composite } \\
\text { depth } \\
\text { (m) }\end{array}$ & $\begin{array}{l}\text { Age } \\
\text { (ka) }\end{array}$ & $\begin{array}{c}\text { Opal } \\
(w t \%)\end{array}$ & $\begin{array}{l}\text { MAR opal } \\
\text { (g/cm² k.y.) }\end{array}$ & $\begin{array}{l}\text { Siliciclastic } \\
\text { fraction } \\
>2 \mu \mathrm{m} \\
(\mathrm{wt} \%)\end{array}$ \\
\hline 104.50 & 2570.2 & 24.73 & 0.38 & 17.15 & 0.27 & 129.37 & 2890.0 & 61.46 & 3.23 & 5.00 \\
\hline 104.73 & 2577.7 & 19.27 & 0.37 & 32.07 & 0.62 & 129.66 & 2892.3 & 58.79 & 2.90 & 5.86 \\
\hline 105.08 & 2587.5 & 35.02 & 0.53 & 11.86 & 0.18 & 129.95 & 2894.6 & 55.00 & 2.80 & 5.70 \\
\hline 105.38 & 2597.4 & 37.75 & 0.52 & 13.07 & 0.18 & 130.24 & 2896.9 & 54.57 & 3.03 & 5.08 \\
\hline 105.67 & 2608.2 & 27.36 & 0.42 & 15.08 & 0.23 & 130.47 & 2898.7 & 61.00 & 3.09 & 4.71 \\
\hline 105.96 & 2619.0 & 17.02 & 0.25 & 40.69 & 0.59 & 130.82 & 2901.5 & 50.72 & 2.23 & 5.31 \\
\hline 106.19 & 2626.2 & 18.43 & 0.50 & 47.70 & 0.29 & 131.11 & 2903.7 & 49.33 & 2.69 & 9.92 \\
\hline 106.54 & 2632.3 & 29.88 & 1.08 & 17.43 & 0.63 & 131.39 & 2905.7 & 50.00 & 3.94 & 5.74 \\
\hline 106.83 & 2637.3 & 23.84 & 0.83 & 19.83 & 0.69 & 131.68 & 2907.5 & 54.38 & 4.36 & 4.89 \\
\hline 107.12 & 2642.4 & 37.40 & 1.17 & 16.39 & 0.51 & 131.97 & 2909.2 & 64.38 & 4.27 & 6.41 \\
\hline 107.41 & 2647.4 & 23.50 & 0.74 & 32.02 & 1.00 & 132.26 & 2910.9 & 70.91 & 5.10 & 6.96 \\
\hline 107.64 & 2652.7 & 19.17 & 0.43 & 34.00 & 0.76 & 132.55 & 2912.7 & 75.64 & 5.42 & 6.78 \\
\hline 108.01 & 2665.2 & 26.09 & 0.48 & 29.22 & 0.54 & 132.84 & 2914.4 & 70.98 & 4.89 & 5.64 \\
\hline 108.29 & 2673.5 & 24.14 & 0.58 & 16.79 & 0.40 & 133.13 & 2916.2 & 45.83 & 3.04 & 3.43 \\
\hline 108.58 & 2679.0 & 32.43 & 0.71 & 10.94 & 0.24 & 133.41 & 2917.9 & 68.20 & 4.52 & 5.04 \\
\hline 108.87 & 2684.4 & 28.39 & 0.77 & 18.30 & 0.49 & 133.83 & 2920.4 & 69.47 & 5.57 & 5.09 \\
\hline 109.10 & 2688.8 & 31.65 & 0.88 & 19.03 & 0.53 & 133.99 & 2921.3 & 57.58 & 3.82 & 5.81 \\
\hline 109.45 & 2695.4 & 17.05 & 0.54 & 39.67 & 1.25 & 134.28 & 2923.1 & 68.44 & 4.92 & 5.67 \\
\hline 109.74 & 2701.0 & 19.54 & 0.58 & 27.69 & 0.82 & 134.49 & 2924.3 & 70.25 & 4.29 & 3.99 \\
\hline 110.03 & 2706.7 & 27.81 & 0.88 & 22.47 & 0.71 & 134.79 & 2926.1 & 60.26 & 4.75 & 4.54 \\
\hline 110.32 & 2712.3 & 32.45 & 0.92 & 15.50 & 0.44 & 135.02 & 2927.5 & 56.00 & 3.97 & 5.83 \\
\hline 110.56 & 2717.0 & 36.28 & 0.99 & 15.69 & 0.43 & 135.38 & 2929.7 & 61.52 & 4.88 & 3.44 \\
\hline 110.91 & 2723.8 & 27.84 & 0.87 & 14.73 & 0.46 & 135.67 & 2931.4 & 69.33 & 4.58 & 3.08 \\
\hline 111.20 & 2729.2 & 32.60 & 1.62 & 12.34 & 0.61 & 135.97 & 2933.2 & 73.46 & 4.37 & 3.32 \\
\hline 111.76 & 2734.3 & 62.48 & 2.61 & 8.39 & 0.35 & 136.27 & 2935.0 & 73.44 & 5.01 & 2.42 \\
\hline 112.06 & 2737.0 & 65.39 & 2.49 & 6.50 & 0.25 & 136.51 & 2936.5 & 71.57 & 4.53 & 3.34 \\
\hline 112.35 & 2739.7 & 68.15 & 2.85 & 5.69 & 0.24 & 136.87 & 2938.5 & 75.58 & 4.46 & 2.77 \\
\hline 112.64 & 2742.3 & 70.88 & 3.09 & 8.48 & 0.37 & 137.15 & 2940.1 & 73.06 & 4.04 & 3.08 \\
\hline 112.95 & 2745.2 & 68.76 & 2.74 & 3.53 & 0.14 & 137.46 & 2941.8 & 74.96 & 4.84 & 3.33 \\
\hline 113.24 & 2747.8 & 63.35 & 2.48 & 3.57 & 0.14 & 137.76 & 2943.5 & 73.96 & 4.68 & 5.65 \\
\hline 113.46 & 2749.8 & 67.54 & 2.96 & 7.20 & 0.32 & 137.99 & 2944.8 & 73.36 & 5.68 & 5.47 \\
\hline 113.80 & 2752.9 & 74.14 & 2.85 & 2.61 & 0.10 & 138.35 & 2946.8 & 69.47 & 5.31 & 10.49 \\
\hline 114.08 & 2756.0 & 76.42 & 2.32 & 2.52 & 0.08 & 138.64 & 2948.4 & 72.91 & 5.99 & 5.16 \\
\hline 114.37 & 2759.1 & 73.23 & 2.27 & 3.28 & 0.10 & 138.95 & 2950.2 & 69.91 & 4.74 & 8.98 \\
\hline 114.66 & 2762.3 & 77.07 & 2.11 & 0.82 & 0.02 & 139.24 & 2951.8 & 58.42 & 3.98 & 7.97 \\
\hline 114.89 & 2764.8 & 78.06 & 2.48 & 2.30 & 0.07 & 139.48 & 2953.1 & 55.21 & 4.28 & 15.96 \\
\hline 115.23 & 2768.5 & 76.64 & 2.44 & 1.56 & 0.05 & 139.84 & 2955.2 & 73.63 & 4.13 & 2.81 \\
\hline 115.50 & 2771.5 & 79.18 & 2.06 & 0.97 & 0.03 & 140.12 & 2956.7 & 74.02 & 4.85 & 3.91 \\
\hline 115.79 & 2774.6 & 72.48 & 2.08 & 1.84 & 0.05 & 140.43 & 2958.5 & 70.63 & 4.97 & 3.78 \\
\hline 116.08 & 2777.8 & 77.04 & 2.13 & 3.61 & 0.10 & 140.74 & 2960.2 & 79.61 & 5.22 & 3.12 \\
\hline 116.31 & 2780.3 & 72.05 & 2.37 & 2.56 & 0.08 & 140.97 & 2961.5 & 65.41 & 4.98 & 4.73 \\
\hline 116.65 & 2784.0 & 76.44 & 2.32 & 3.73 & 0.11 & 141.32 & 2963.5 & 42.97 & 3.83 & 4.10 \\
\hline 116.92 & 2786.4 & 73.35 & 3.43 & 2.34 & 0.11 & 141.60 & 2965.0 & 45.95 & 5.18 & 7.73 \\
\hline 117.22 & 2788.6 & 71.06 & 3.76 & 7.62 & 0.40 & 141.90 & 2966.7 & 39.27 & 4.32 & 5.68 \\
\hline 117.50 & 2790.7 & 74.37 & 3.79 & 2.29 & 0.12 & 142.19 & 2968.3 & 39.85 & 4.62 & 1.32 \\
\hline 117.73 & 2792.4 & 77.58 & 4.26 & 2.35 & 0.13 & 142.47 & 2969.9 & 47.87 & 4.82 & 6.41 \\
\hline 118.07 & 2795.0 & 72.42 & 3.67 & 4.58 & 0.23 & 142.76 & 2971.5 & 53.67 & 5.32 & 6.98 \\
\hline 118.35 & 2797.1 & 67.80 & 3.00 & 11.79 & 0.52 & 143.04 & 2973.1 & 52.88 & 4.71 & 4.38 \\
\hline 118.64 & 2799.3 & 65.02 & 3.82 & 13.33 & 0.78 & 143.33 & 2974.7 & 60.32 & 5.36 & 3.69 \\
\hline 118.92 & 2801.4 & 72.07 & 3.51 & 2.84 & 0.14 & 143.62 & 2976.4 & 62.92 & 5.21 & 4.12 \\
\hline 119.15 & 2803.1 & 65.95 & 3.73 & 3.43 & 0.19 & 143.87 & 2977.8 & 62.09 & 4.57 & 3.90 \\
\hline 119.23 & 2803.7 & 76.54 & 3.63 & 3.25 & 0.15 & 144.15 & 2979.3 & 63.80 & 4.93 & 3.37 \\
\hline 119.49 & 2805.7 & 68.54 & 3.32 & 2.62 & 0.13 & 144.51 & 2981.4 & 68.96 & 3.93 & 4.58 \\
\hline 119.77 & 2807.8 & 67.37 & 3.10 & 3.52 & 0.16 & 145.11 & 2985.2 & 67.53 & 3.91 & 2.99 \\
\hline 120.06 & 2809.9 & 66.04 & 2.76 & 2.87 & 0.12 & 145.41 & 2987.1 & 58.76 & 3.67 & 5.06 \\
\hline 120.37 & 2812.5 & 68.06 & 2.84 & 5.72 & 0.24 & 145.65 & 2988.6 & 57.43 & 4.42 & 3.42 \\
\hline 120.62 & 2814.6 & 77.22 & 3.38 & 5.18 & 0.23 & 146.01 & 2990.9 & 45.59 & 3.64 & 3.68 \\
\hline 120.91 & 2817.0 & 77.41 & 3.39 & 4.34 & 0.19 & 146.31 & 2992.8 & 44.29 & 3.45 & 6.49 \\
\hline 121.19 & 2819.3 & 71.13 & 3.25 & 5.93 & 0.27 & 146.61 & 2994.7 & 50.81 & 4.16 & 3.94 \\
\hline 121.57 & 2822.5 & 46.54 & 2.32 & 9.28 & 0.46 & 146.91 & 2996.6 & 61.99 & 4.20 & 2.74 \\
\hline 121.76 & 2824.1 & 70.81 & 2.96 & 5.89 & 0.25 & 147.15 & 2998.2 & 53.16 & 3.75 & 3.28 \\
\hline 122.05 & 2826.5 & 69.53 & 2.76 & 6.76 & 0.27 & 147.51 & 3000.5 & 58.48 & 3.81 & 3.91 \\
\hline 122.33 & 2828.8 & 67.33 & 2.95 & 3.41 & 0.15 & 147.81 & 3002.4 & 64.13 & 4.02 & 2.77 \\
\hline 122.62 & 2831.3 & 64.43 & 3.08 & 5.05 & 0.24 & 148.11 & 3004.3 & 64.71 & 4.44 & 3.11 \\
\hline 122.90 & 2833.6 & 64.69 & 3.09 & 5.31 & 0.25 & 148.41 & 3006.2 & 62.92 & 3.85 & 3.97 \\
\hline 122.90 & 2833.6 & 63.64 & 2.77 & 5.36 & 0.23 & 148.65 & 3007.7 & 66.67 & 4.81 & 3.75 \\
\hline 123.19 & 2836.0 & 64.30 & 3.07 & 6.09 & 0.29 & 149.01 & 3009.8 & 62.30 & 4.10 & 4.40 \\
\hline 123.52 & 2838.8 & 60.04 & 2.84 & 2.58 & 0.12 & 149.31 & 3011.7 & 62.34 & 4.16 & 4.70 \\
\hline 123.83 & 2841.3 & 61.67 & 3.10 & 4.47 & 0.22 & 149.61 & 3013.5 & 62.42 & 3.71 & 6.00 \\
\hline 124.12 & 2843.8 & 64.88 & 2.77 & 5.41 & 0.23 & 149.91 & 3015.3 & 64.42 & 4.51 & 4.53 \\
\hline 124.41 & 2846.4 & 62.09 & 2.42 & 2.83 & 0.11 & 150.15 & 3016.7 & 61.26 & 5.64 & 5.20 \\
\hline 124.64 & 2848.5 & 72.01 & 2.99 & 3.87 & 0.16 & 150.51 & 3018.9 & 63.09 & 3.80 & 5.49 \\
\hline 125.00 & 2851.8 & 64.08 & 2.95 & 4.16 & 0.19 & 150.81 & 3020.7 & 67.54 & 4.97 & 8.09 \\
\hline 125.29 & 2854.5 & 61.92 & 2.66 & 5.49 & 0.24 & 151.11 & 3022.6 & 60.63 & 4.36 & 13.21 \\
\hline 125.58 & 2857.1 & 69.00 & 2.82 & 5.57 & 0.23 & 151.65 & 3025.8 & 67.39 & 5.73 & 4.74 \\
\hline 125.87 & 2859.8 & 66.44 & 2.73 & 4.01 & 0.16 & 152.12 & 3028.7 & 68.67 & 4.67 & 4.71 \\
\hline 126.10 & 2861.9 & 68.33 & 2.73 & 3.41 & 0.14 & 152.51 & 3031.0 & 65.59 & 4.72 & 3.88 \\
\hline 126.45 & 2865.1 & 74.96 & 3.11 & 3.33 & 0.14 & 153.14 & 3034.5 & 68.61 & 4.00 & 4.50 \\
\hline 126.74 & 2867.8 & 85.62 & 3.32 & 10.12 & 0.39 & 153.55 & 3036.9 & 43.60 & 2.67 & 2.29 \\
\hline 127.03 & 2870.5 & 67.92 & 2.94 & 3.28 & 0.14 & 153.90 & 3038.9 & 64.54 & 4.72 & 4.94 \\
\hline 127.33 & 2873.2 & 56.64 & 2.53 & 4.19 & 0.19 & 154.13 & 3040.2 & 66.03 & 4.82 & 4.03 \\
\hline 127.56 & 2875.3 & 49.37 & 2.71 & 3.90 & 0.21 & 154.49 & 3042.2 & 66.02 & 5.02 & 3.74 \\
\hline 127.91 & 2878.5 & 49.96 & 3.38 & 5.09 & 0.34 & 154.77 & 3043.8 & 69.61 & 4.68 & 4.52 \\
\hline 128.20 & 2880.8 & 52.11 & 3.07 & 3.22 & 0.19 & 155.07 & 3045.5 & 68.47 & 4.40 & 3.46 \\
\hline 128.49 & 2883.1 & 70.78 & 3.79 & 4.89 & 0.26 & 155.37 & 3047.2 & 69.03 & 4.43 & 4.69 \\
\hline 128.78 & 2885.3 & 57.51 & 3.08 & 4.37 & 0.23 & 155.60 & 3048.5 & 74.07 & 4.54 & 4.22 \\
\hline 129.02 & 2887.2 & 66.42 & 3.21 & 5.40 & 0.26 & 155.96 & 3050.5 & 73.29 & 4.71 & 4.02 \\
\hline
\end{tabular}


G.H. HAUG ET AL.

Table 2 (continued).

\begin{tabular}{|c|c|c|c|c|c|}
\hline $\begin{array}{l}\text { Composite } \\
\text { depth } \\
\text { (m) }\end{array}$ & $\begin{array}{l}\text { Age } \\
\text { (ka) }\end{array}$ & $\begin{array}{l}\text { Opal } \\
(w t \%)\end{array}$ & $\begin{array}{c}\text { MAR opal } \\
\left(\mathrm{g} / \mathrm{cm}^{2} \text { k.y. }\right)\end{array}$ & $\begin{array}{l}\text { Siliciclastic } \\
\text { fraction } \\
>2 \mu \mathrm{m} \\
(w t \%)\end{array}$ & $\begin{array}{l}\text { MAR silici- } \\
\text { clastic fraction } \\
>2 \mu \mathrm{m} \\
\left(\mathrm{g} / \mathrm{cm}^{2} \mathrm{k} . \mathrm{y} .\right)\end{array}$ \\
\hline 156.24 & 3052.1 & 72.38 & 4.44 & 4.43 & 0.27 \\
\hline 156.54 & 3053.8 & 76.24 & 4.03 & 3.36 & 0.18 \\
\hline 156.84 & 3055.7 & 70.93 & 3.95 & 3.90 & 0.22 \\
\hline 157.07 & 3057.1 & 69.53 & 4.04 & 4.91 & 0.29 \\
\hline 157.42 & 3059.3 & 73.29 & 5.21 & 4.02 & 0.29 \\
\hline 157.71 & 3061.2 & 65.66 & 3.81 & 8.20 & 0.48 \\
\hline 158.01 & 3063.1 & 56.78 & 3.29 & 4.77 & 0.28 \\
\hline 158.31 & 3064.9 & 68.96 & 3.81 & 5.01 & 0.28 \\
\hline 158.54 & 3066.4 & 67.72 & 4.28 & 3.46 & 0.22 \\
\hline 158.89 & 3068.6 & 66.71 & 4.58 & 4.06 & 0.28 \\
\hline 159.18 & 3070.4 & 57.04 & 4.37 & 6.68 & 0.51 \\
\hline 159.48 & 3072.3 & 58.16 & 3.83 & 10.29 & 0.68 \\
\hline 159.77 & 3074.1 & 61.86 & 3.69 & 3.61 & 0.22 \\
\hline 160.01 & 3076.0 & 56.78 & 2.25 & 5.36 & 0.21 \\
\hline 160.36 & 3079.4 & 46.84 & 2.49 & 5.00 & 0.27 \\
\hline 160.65 & 3082.3 & 52.15 & 2.55 & 4.10 & 0.20 \\
\hline 160.95 & 3085.2 & 56.64 & 3.06 & 5.58 & 0.30 \\
\hline 161.37 & 3089.4 & 56.91 & 2.84 & 3.19 & 0.16 \\
\hline 161.72 & 3092.8 & 54.72 & 2.54 & 3.60 & 0.17 \\
\hline 162.25 & 3098.0 & 59.21 & 2.94 & 2.81 & 0.14 \\
\hline 162.67 & 3102.2 & 56.78 & 2.72 & 3.24 & 0.16 \\
\hline 163.13 & 3106.7 & 35.79 & 1.26 & 2.18 & 0.08 \\
\hline 163.42 & 3109.6 & 64.07 & 3.19 & 3.45 & 0.17 \\
\hline 163.86 & 3113.9 & 67.14 & 3.11 & 2.69 & 0.12 \\
\hline 164.30 & 3118.2 & 67.63 & 3.45 & 2.66 & 0.14 \\
\hline 164.65 & 3121.7 & 65.90 & 2.92 & 2.97 & 0.13 \\
\hline 165.14 & 3126.5 & 66.89 & 3.10 & 3.99 & 0.19 \\
\hline 165.43 & 3129.4 & 67.65 & 2.77 & 3.47 & 0.14 \\
\hline 165.72 & 3132.2 & 66.91 & 3.25 & 3.27 & 0.16 \\
\hline 166.01 & 3135.1 & 62.35 & 2.75 & 3.74 & 0.17 \\
\hline 166.24 & 3137.3 & 68.86 & 3.25 & 4.11 & 0.19 \\
\hline 166.59 & 3140.8 & 49.04 & 2.02 & 2.76 & 0.11 \\
\hline 166.88 & 3143.6 & 72.94 & 3.22 & 4.67 & 0.21 \\
\hline 167.18 & 3146.4 & 72.45 & 3.78 & 3.91 & 0.20 \\
\hline 167.47 & 3148.5 & 67.54 & 4.28 & 4.10 & 0.26 \\
\hline 167.70 & 3150.1 & 65.40 & 4.12 & 4.69 & 0.30 \\
\hline 168.34 & 3154.7 & 52.01 & 3.31 & 4.29 & 0.27 \\
\hline 168.63 & 3156.7 & 59.92 & 4.01 & 3.50 & 0.23 \\
\hline 168.93 & 3158.8 & 53.84 & 3.65 & 4.28 & 0.29 \\
\hline 169.80 & 3165.0 & 62.40 & 4.07 & 2.31 & 0.15 \\
\hline 170.09 & 3167.1 & 64.86 & 2.95 & 2.35 & 0.11 \\
\hline 170.38 & 3170.1 & 61.41 & 2.58 & 2.61 & 0.11 \\
\hline 170.61 & 3172.5 & 56.59 & 2.50 & 5.95 & 0.26 \\
\hline 170.96 & 3176.1 & 63.82 & 2.57 & 5.46 & 0.22 \\
\hline 171.26 & 3179.2 & 66.50 & 2.35 & 3.99 & 0.14 \\
\hline 171.55 & 3182.2 & 67.07 & 2.37 & 5.39 & 0.19 \\
\hline 171.84 & 3185.1 & 67.42 & 2.62 & 4.77 & 0.19 \\
\hline 172.07 & 3187.5 & 39.15 & 1.47 & 3.91 & 0.15 \\
\hline 172.42 & 3191.1 & 58.20 & 2.27 & 4.31 & 0.17 \\
\hline 172.71 & 3194.1 & 59.45 & 1.39 & 4.93 & 0.11 \\
\hline
\end{tabular}


EVOLUTION OF SEDIMENTATION PATTERNS

Table 3. Oxygen isotope data from Site 882.

\begin{tabular}{|c|c|c|c|c|c|c|c|}
\hline $\begin{array}{l}\text { Composite } \\
\text { depth } \\
\text { (m) }\end{array}$ & $\begin{array}{l}\text { Age } \\
\text { (ka) }\end{array}$ & N. pachyderma (I) & Uvigerina spp. & $\begin{array}{l}\text { Composite } \\
\text { depth } \\
\text { (m) }\end{array}$ & $\begin{array}{l}\text { Age } \\
\text { (ka) }\end{array}$ & N. pachyderma (1) & Uvigerina spp. \\
\hline 0.30 & 4.9 & 2.57 & 4.02 & 24.81 & 475.3 & 4.21 & \\
\hline 0.60 & 9.8 & 2.99 & 4.57 & 25.08 & 479.1 & 3.98 & \\
\hline 0.90 & 14.7 & 3.98 & 5.08 & 25.67 & 487.6 & 3.08 & 4.35 \\
\hline 1.20 & 20.1 & 3.96 & 5.11 & 25.97 & 491.9 & 3.24 & 3.91 \\
\hline 1.55 & 26.4 & 3.60 & & 26.26 & 496.0 & 2.71 & 3.83 \\
\hline 1.85 & 31,9 & 3.82 & 5.18 & 26.28 & 496.3 & 2.44 & 3.93 \\
\hline 2.10 & 36.4 & 3.46 & 3.94 & 26.43 & 498.5 & 2.66 & 4.01 \\
\hline 2.36 & 41.1 & 3.55 & 4.49 & 26.55 & 500.3 & 2.69 & 3.97 \\
\hline 2.52 & 44.0 & 3.40 & & 26.82 & 504.2 & 2.82 & 4.28 \\
\hline 2.66 & 46.6 & 3.72 & 4.59 & 27.44 & 513.3 & 2.95 & 4.03 \\
\hline 2.96 & 52.0 & 3.98 & 4.43 & 27.67 & 520.1 & 2.85 & 4.24 \\
\hline 3.05 & 53.7 & 3.37 & 4.57 & 27.75 & 523.8 & 2.90 & 4.25 \\
\hline 3.20 & 56.4 & 3.26 & 4.42 & 28.00 & 535.3 & 3.33 & 4.72 \\
\hline 4.46 & 77.4 & 3.37 & & 28.16 & 542.7 & 3.04 & 4.65 \\
\hline 4.70 & 80.9 & 3.50 & 4.02 & 28.32 & 548.4 & 2.56 & 4.77 \\
\hline 5.06 & 86.1 & 3.11 & & 28.61 & 557.5 & 3.57 & 4.74 \\
\hline 7.46 & 132.5 & 2.37 & & 29.12 & 579.1 & 2.60 & 4.05 \\
\hline 7.67 & 138.6 & 2.28 & & 29.36 & 590.8 & 2.44 & \\
\hline 7.70 & 139.1 & 2.47 & 3.48 & 29.55 & 598.5 & & 3.69 \\
\hline 8.06 & 146.1 & 3.58 & & 29.80 & 608.6 & 2.52 & 3.45 \\
\hline 8.36 & 151.9 & 4.00 & & 30.04 & 613.0 & 4.08 & 4.55 \\
\hline 9.20 & 164.4 & 3.48 & 4.85 & 30.28 & 617.1 & 2.67 & 4.52 \\
\hline 9.69 & 169.4 & 3.75 & & 30.53 & 621.5 & 3.08 & \\
\hline 9.93 & 171.9 & 3.38 & & 30.77 & 625.6 & 2.32 & \\
\hline 10.45 & 177.3 & 4.07 & & 31.00 & 629.6 & 2.93 & \\
\hline 13.40 & 245.1 & 3.51 & & 31.74 & 645.2 & 3.33 & 4.29 \\
\hline 13.65 & 251.2 & 3.41 & 3.79 & 31.98 & 649.2 & & \\
\hline 13.97 & 256.9 & & 5.16 & 32.20 & 652.9 & 4.06 & 5.24 \\
\hline 14.27 & 262.2 & 5.74 & & 32.49 & 657.8 & 3.92 & \\
\hline 14.35 & 263.6 & 3.38 & 4.62 & 32.73 & 661.8 & 4.42 & 5.13 \\
\hline 14.56 & 267.4 & 4.11 & 3.40 & 33.08 & 667.6 & 3.67 & \\
\hline 14.69 & 269.7 & 3.33 & 4.65 & 33.37 & 672.4 & 3.38 & \\
\hline 15.07 & 276.4 & 2.67 & & 33.67 & 677.4 & 3.44 & \\
\hline 16.31 & 297.4 & 3.69 & 4.19 & 33.96 & 682.1 & 3.66 & \\
\hline 17.47 & 324.0 & 3.96 & & 34.20 & 685.7 & 2.56 & \\
\hline 17.77 & 331.2 & 4.18 & & 34.55 & 691.0 & 2.93 & \\
\hline 18.63 & 349.4 & 3.93 & & 34.84 & 695.4 & 3.30 & 4.03 \\
\hline 18.92 & 354.1 & 5.02 & 5.31 & 35.14 & 700.0 & 2.97 & 3.75 \\
\hline 21.56 & 407.1 & 4.00 & 4.19 & 35.44 & 704.5 & 2.37 & 3.76 \\
\hline 21.78 & 411.5 & 1.89 & 3.28 & 35.67 & 708.0 & 2.57 & \\
\hline 21.87 & 413.2 & 1.68 & 3.29 & 36.02 & 713.3 & 2.94 & \\
\hline 22.14 & 418.5 & 2.27 & 3.53 & 36.31 & 717.7 & 3.16 & 4.18 \\
\hline 22.26 & 420.8 & 2.26 & 3.84 & 36.91 & 729.9 & 3.80 & 3.64 \\
\hline 22.66 & 428.5 & 2.99 & 4.41 & 37.14 & 736.3 & 3.28 & 4.50 \\
\hline 22.73 & 429.8 & 4.09 & 4.54 & 37.50 & 746.3 & 3.15 & 4.39 \\
\hline 23.03 & 435.6 & 3.68 & & 37.78 & 754.1 & 4.56 & 4.74 \\
\hline 23.34 & 441.5 & 4.55 & & 38.08 & 762.0 & 4.03 & \\
\hline 23.62 & 446.8 & 3.65 & & 38.38 & 769.3 & 7.41 & \\
\hline 24.18 & 459.7 & & 4.60 & 38.97 & 781.5 & 2.96 & \\
\hline 24.20 & 460.2 & 2.83 & 4.44 & 39.23 & 786.0 & 2.80 & 3.46 \\
\hline 24.50 & 468.2 & 3.28 & & & & & \\
\hline
\end{tabular}




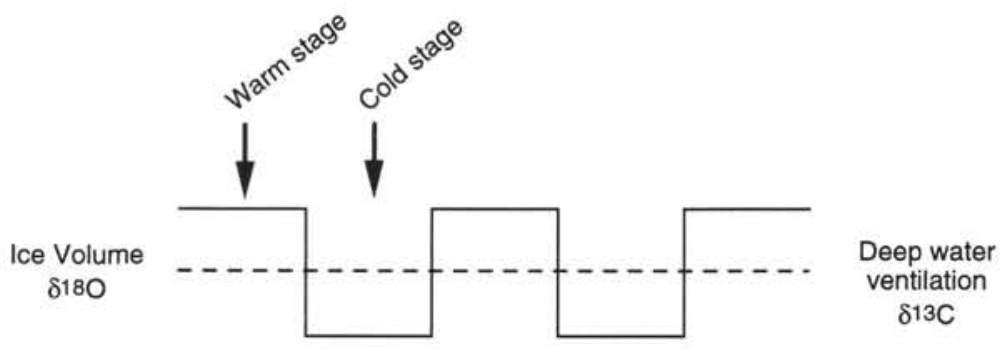

A

Conveyor strength

(NADW formation)

Strong Weak

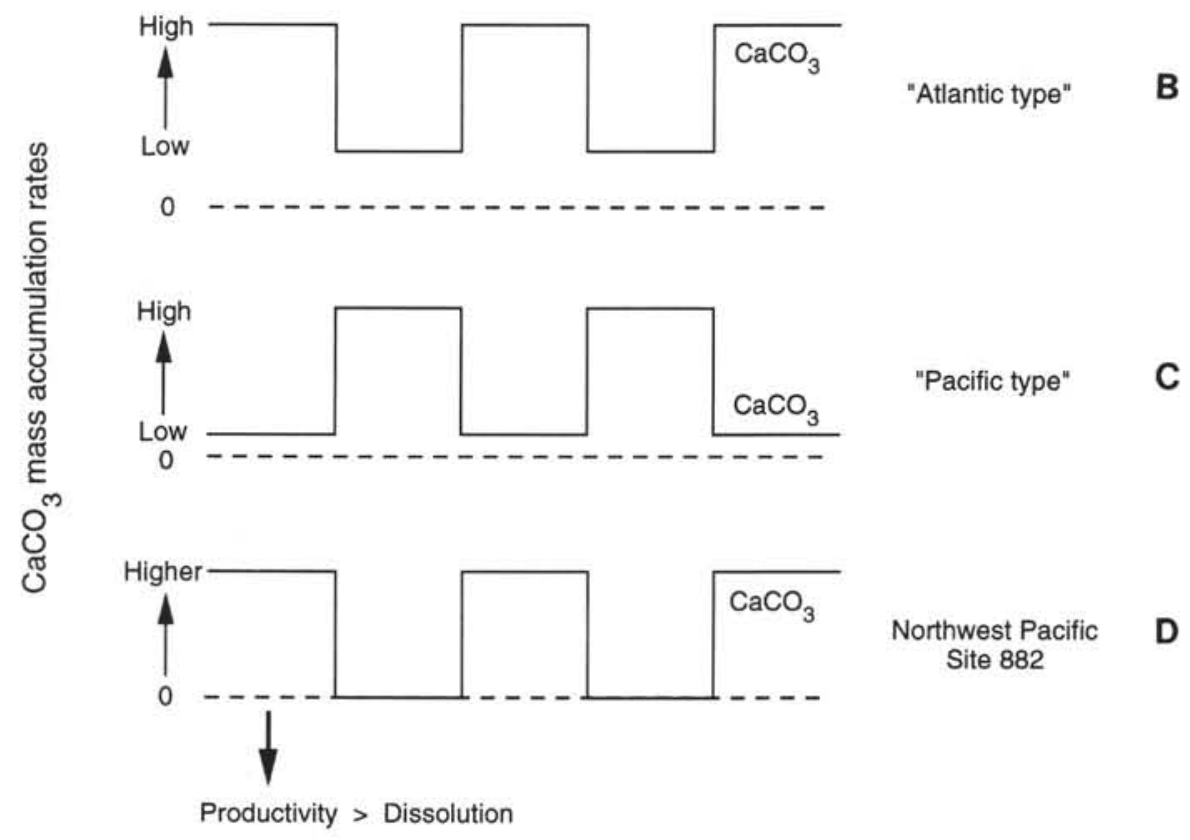

Figure 4. A-D. Summary figure of the global carbonate accumulation patterns in the Atlantic and Pacific oceans in comparison with northwest Pacific Site 882 on a glacial (cold stage) to interglacial (warm stage) time scale. The different patterns are controlled by the variability of the global deep-water salinity conveyor belt. 